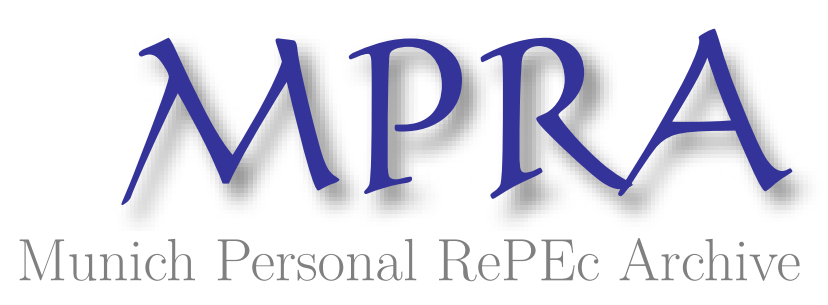

\title{
Construction in Italy's regions, 1861-1913
}

Ciccarelli, Carlo and Fenoaltea, Stefano

Università di Roma "Tor Vergata"

2008

Online at https://mpra.ub.uni-muenchen.de/9714/

MPRA Paper No. 9714, posted 24 Jul 2008 10:33 UTC 
CONSTRUCTION IN ITALY'S REGIONS, 1861-1913

\author{
Carlo Ciccarelli \\ and \\ Stefano Fenoaltea \\ Facoltà di Economia \\ Università di Roma "Tor Vergata" \\ stefano.fenoaltea@uniroma2.it \\ carlo.ciccarelli@uniroma2.it
}

2008

The regional estimates were produced as part of the research project on "Unità d'Italia e sviluppo disuguale: la struttura creditizia e la crescita industriale per regioni dal 1861 al 1913" of the Ufficio Ricerche Storiche of the Bank of Italy. The authors alone are responsible for the material presented here. 
CONSTRUCTION IN ITALY'S REGIONS, 1861-1913

\begin{abstract}
This paper presents time-series estimates of construction activity in the regions of postUnification Italy. Total construction followed very different time paths, reflecting the sharply local cycles in railway construction. Other public works were less idiosyncratic; the boom of the Giolitti years was widely diffused, but that of the 1880s was much more concentrated in Latium and Liguria. In the construction of buildings, the Giolittian boom was marked in the North and Center, but spotty in the South and major islands; earlier swings were comparatively minor, save of course for the 1880s bubble in Latium. Over the long term, railway construction was, per-capita, relatively evenly spread. Other social-overhead construction displays a similar pattern, but with exceptionally high levels in Latium and Liguria. Building construction seems instead to have declined somewhat from North to South; Liguria was again the overall leader, with Latium second.
\end{abstract}

\title{
LE COSTRUZIONI NELLE REGIONI D'ITALIA, 1861-1913
}

\begin{abstract}
Sommario
Questo saggio presenta stime annuali della produzione delle industrie delle costruzioni nelle regioni dell'Italia post-Unitaria. Le costruzioni complessive manifestano andamenti ciclici diversi, indotti dagli sfasamenti delle costruzioni ferroviarie. Le altre opere pubbliche erano meno idiosincratiche: l'ascesa giolittiana è stata alquanto diffusa, quella depretisiana più concentrata nel Lazio e nella Liguria. Nella costruzione di edifici l'ascesa giolittiana si manifesta molto più nettamente nel Centro-Nord che nel Mezzogiorno; nel periodo precedente spicca il ciclo del Lazio. In complesso la distribuzione delle costruzioni ferroviarie appare, pro capite, abbastanza uniforme--e così pure le altre opere pubbliche, eccezion fatta per i picchi raggiunti nel Lazio e in Liguria. Le costruzioni di edifici tende invece a diminuire da Nord a Sud; ancora una volta si trova in testa la Liguria, seguita dal Lazio.
\end{abstract}


CONSTRUCTION IN ITALY'S REGIONS, 1861-1913

\section{Introduction}

This paper presents regional time series estimates of construction activity in postUnification Italy. Construction is relatively well documented by year-specific data, mainly from public documents. These (comparatively) high-frequency data permit the identification of local cycles, which at times differ sharply from region to region.

The most markedly local cycles were in, and due to, railway construction. Railway projects were large, and locally concentrated in time, but not simultaneous: the trunk lines were extended from region to region as the rail-heads advanced, and the network of secondary lines was not uniformly distributed. Other public works were less idiosyncratic; the boom of the Giolitti years was widely diffused, but that of the 1880s was much more concentrated in Latium and Liguria. In the construction of buildings, finally, the Giolittian boom was marked in the North and Center, and sharpest in Liguria; it was spottier in the South and major islands, and missed altogether by the Abruzzi, where emigration was particularly heavy. Earlier swings are comparatively minor, save of course for the 1880s bubble in Latium.

Over the long term, on a per-capita basis railway construction was relatively evenly spread. Other social-overhead construction displays a similar pattern, but with two upside outliers; these favored recipients of public works were Latium (and more specifically, of course, the capital city of Rome), and even more so, surprisingly, Liguria. Building construction seems instead to have declined somewhat from North to South; Liguria was again the overall leader, and Latium again a favored outlier.

\section{Sources and methods}

The regional time series presented here refer to buildings, railways, other socialoverhead capital, and total construction; the new-construction series are collected in Appendix A, the maintenance series in Appendix B. The corresponding national series were presented many years ago. ${ }^{\mathrm{i}}$. These too have been marginally updated; the revised aggregates are presented in Appendix C. ${ }^{\text {ii }}$ All these series measure construction by value added at (national) 1911 prices; the estimated ratio of value added to value equals .34 for new buildings, .51 for other new construction, and .60 for maintenance.

The regional series are derived essentially from the same sources, and with the same methods, as their national counterparts, which have already been described in some detail. ${ }^{\text {iii }}$ The building estimates are derived in the main from the annual changes in the buildings-tax base, allowing for the lags between construction and assessment; but the tax-base information is unfortunately limited. On the one hand, it omits rural buildings (subject instead to the land tax); like the national estimates, the regional estimates inflate taxed construction into total construction with an eye to the relative changes in the population housed in taxed structures, and in exempt rural structures, from one census to the next. On the other hand, the tax-base evidence yields estimates of construction only from 1872. Like the national estimates, the regional estimates extrapolate the series back to 1861 with the aid of the index of urban construction (derived from the consumption of construction materials subject to municipal tax); the problem here is that not all the regions are present in the national sample. Most regional extrapolations in the South and Center rely perforce on the national index: the estimates of building construction for the initial decade are best guesses, but contain little local evidence, and should be used with caution.

The railway construction series are of relatively high quality. Their largest component is based directly on highly detailed evidence on the mileage of newly opened lines, here 
distributed over the period of construction. New construction for improvements, and maintenance work, are typically not documented at the local level; the present estimates allocate the national totals on the basis of traffic.

Other social-overhead construction is estimated, in the main, from public budgets, complemented by estimates of privately financed work (mostly for the utilities). The data used for the national estimates typically carry a regional tag in the sources themselves, and the reconstruction of the regional flows is correspondingly straightforward. Absent regional cost indices, the regional current-price series are deflated by the national price indices; these common deflators introduce some common movements that are negligible in the case of new construction, but perceptible in that of (trend-dominated) maintenance. ${ }^{\text {iv }}$

\section{Regional construction: levels}

Table 1 presents summary estimates of total construction (that is, new construction plus maintenance). Cols. 1 - 4 report the regional averages from 1861 to 1913 for buildings, railways, other social-overhead capital, and their sum. Col. 5 reports the (here) standard measure of scale, that is, the regions' male population of working age in 1901; and cols. 6 - 9 report the per-man equivalents of cols. 1 - 4. These last numbers, scaled by the corresponding national averages, are illustrated in Map 1.

In none of these three broad categories did regional construction per man average as little as half, or as much as twice, the national average. The distribution of railway construction was even tighter than that, with no region reaching so much as 1.5 times the national average. Building construction and other social-overhead construction are characterized by a similarly tight distribution, save for two up-side outliers: the same two, in fact, Liguria and Latium.

Building construction seems tied not just to demographic growth, but specifically to urban/industrial growth: this may be why it was so much higher in Liguria and Latium (and even Lombardy) than in Apulia and Sicily, where overall demographic growth was close to Liguria's and higher than in any other region. ${ }^{v}$ Non-railway public works were disproportionately lavished on Latium, with the capital city of Rome; and Liguria alone captured almost a third of Italy's military construction, mainly for the naval base at La Spezia, boosting the region's (non-building, non-railway) total by a third. ${ }^{\mathrm{vi}}$

In total construction, on the present metric, Liguria scores highest, followed closely by Latium. Liguria's population density was extremely high, and in a sense the per-man measure limits that region's advantage. If the figures in Table 1 are recalculated per square kilometer rather than per man, Liguria's average balloons to three times Italy's, with the runner-up near half that.

Figure 1 is an alternative illustration of the estimates that yield Map 1. It confirms the suggestion that (outliers apart) the average construction of buildings alone tended to decline as one moved from North to South; on the present metric, railway and other social-overhead construction do not seem to share this geographic bias.

\section{Regional construction: paths}

Figures 2 - 5 illustrate the corresponding time series (again covering maintenance and new construction together), again scaled by the regions' male population of working age in 1901.

In Figure 2, which refers to buildings, Liguria and Latium again stand out. Construction there varied more than elsewhere. In Liguria, at the end of the period at hand, it reached levels without precedent or parallel; in Latium, and Latium alone, the sharp cycle of the 1880s is also apparent. The sustained upswing of the Giolitti years is also evident in the other major regions of the North and Center. The upswing came very late in much of the continental South, in 
Sicily (thanks in part to the earthquake of 1908) and in Sardinia; in the Abruzzi, where population was then rapidly falling, it never came at all.

Figure 3, which refers to railway construction, reveals a variety of patterns. Many regions display a local peak when the trunks were built. In the early years one can readily trace the extension of the rail line from Bologna to and down the Adriatic coast and then along the Ionic coast, the connection of the Roman and Tuscan systems across Umbria, and the construction of the Ligurian coast line; the later construction of the Sicilian and Sardinian trunks, of the link from Naples to Apulia across Basilicata, and of the final west-coast trunk in Calabria is similarly evident. In a number of Southern regions, and in Umbria, there was little else: these sharp new-construction peaks apart, the railway total coincides with the maintenance trend. The wave of secondary-line (and suburban tramway) construction in the 1880s is apparent in most Northern regions, the main Central regions (and especially Latium), and Campania; the late railway-improvement (and urban-tramway-construction) boom is even more specific to the North and the major regions of the Center.

Figure 4 refers in turn to other social overhead capital. Specific episodes again stand out. In Piedmont, for example, the early peak is due to the Cavour canal, the late one (as in Umbria) to hydroelectric construction; in Lombardy, the sharp peak in the 1880 s is due to municipal expenditure to embellish Milan. The main example here is the Apulian aqueduct: it accounts for the late peak in Apulia, and for the even sharper peak in Basilicata, crossed by the main conduit from the headwaters of the Sele in Campania. In the large, the Giolittian boom is again relatively widespread; but the two standouts are again Liguria and Latium, which alone reach high levels in the 1880s as well as at the end. Both regions owe their high overall average (Table 1 and Map 1) to their exceptional performance in the 1880s; and in both that performance seems due to a combination of high State spending, including military spending, and local-government projects.

Figure 5 combines the preceding graphs to illustrate the path of total regional construction (per man), and the role of its components. Over the nineteenth century, the local swings in construction were due overwhelmingly to the bursts of railway work; the only significant exceptions are the above-cited cases of the Cavour canal in Piedmont, of Milan's municipal works in Lombardy, and the 1880s in Liguria and Latium. In the early twentieth century, railway work contributed significantly to the swings in total construction only in exceptional cases (Piedmont, Venetia, Tuscany); the late upswing was driven almost everywhere by other public works, and by the (nationally) unprecedented boom in residential construction.

\section{Major-area construction}

Italy' regions are traditionally grouped into three major areas comprising, respectively, the North-West, the Center/North-East, and the South (and islands). ${ }^{\text {vii }}$ Figures 6 - 9 illustrate, by major area, the construction of buildings, railways, other social-overhead capital, and total construction. The upper graphs illustrate value added at 1911 prices (as obtained directly from Appendices A and B, without further scaling); the lower graphs, the area shares of the national total.

Perhaps the most striking aspect of the value-added graphs is the extent to which local idiosyncrasies are reabsorbed: the regional series often differ, but the macro-area series are broadly parallel, and their movements are altogether closer to those of the national aggregate. This convergence-by-aggregation is particularly noticeable in the case of railway (and total) construction, but present in other categories as well; in fact, the only significant exception is the failure of the North-West to register the building-construction cycle of the 1880s.

The macro-area value-added cycles are relatively similar, but not quite synchronized; 
the macro-area share series are correspondingly unstable, and vary sharply from year to year.

Over the longer-term, the macro-area shares of building construction (Figure 6) point to a secular rise in the share of the Center/North-East, and a one-time increase, around the turn of the century, in the share of the North-West. The share of the South correspondingly declined over time; it dropped especially sharply around the turn of the century, as building construction picked up in the South a full decade after it picked up in the North and Center.

In railway construction (Figure 7) there was something of a regime change: the South's share was tendentially the largest until the end of the nineteenth century, and then much the smallest. As noted above, this seems tied to the switch from initial construction--which favored the South because North and Center were, in 1861, far better equipped--to construction to improve the existing lines where traffic growth had rendered them inadequate.

Other social-overhead construction is illustrated in Figure 8. Excluding short-lived swings (as in the early years, and again in the 1880s), the North-West was generally a poor third, but with a tendency to grow; the South and especially the Center/North-East were typically much higher, but the shares of both these major areas slowly drifted down.

In the aggregate (Figure 9), finally, over the long term the share of the Center/NorthEast tended to remain high, or even to rise slightly. The South's share long matched or exceeded that of the Center/North-East, but then slipped to become a poor third; conversely, the share of the North-West remained the smallest of the three into the 1890s, but then climbed smartly and overtook that of the South. 


\section{NOTES}

i. S. Fenoaltea, "Railway Construction in Italy, 1861-1913," Rivista di storia economica I, International issue (1984), pp. 27-58; Id., "Public Works Construction in Italy, 1861-1913," Rivista di storia economica III, International issue (1986), pp. 1-33; Id., "Construction in Italy, 1861-1913," Rivista di storia economica IV, International issue (1987), pp. 21-53.

ii. The most significant modifications stem from the revision of the construction estimates related to the utilities' infrastructure; see C. Ciccarelli and S. Fenoaltea, "The Growth of the Utilities Industries in Italy's Regions, 1861-1913," Rivista di storia economica, in press. Unlike the national series, the regional series do not distinguish between public and private buildings.

iii. See the papers cited in note 1 above. A full account of the derivation of the national and regional estimates is available on request.

iv. For further details on the railway and other-overhead-capital estimates, and further series that separate civil and military or naval construction, see C. Ciccarelli and S. Fenoaltea, "Social-Overhead Construction in Italy's Regions, 1861-1913," Research in Economic History, forthcoming.

v. Latium obviously also benefited from a super-normal share of public buildings, which themselves represented over a tenth of the total. Income growth may also have mattered, of course, but this evidence is not in yet.

vi. Ciccarelli and Fenoaltea, "Social-Overhead," Table 1.

vii. With the regions listed as in Table 1 , the three major areas correspond respectively to the first three, the next six, and the residual seven. 
Table 1

Regional construction: annual averages

(value added at 1911 prices)

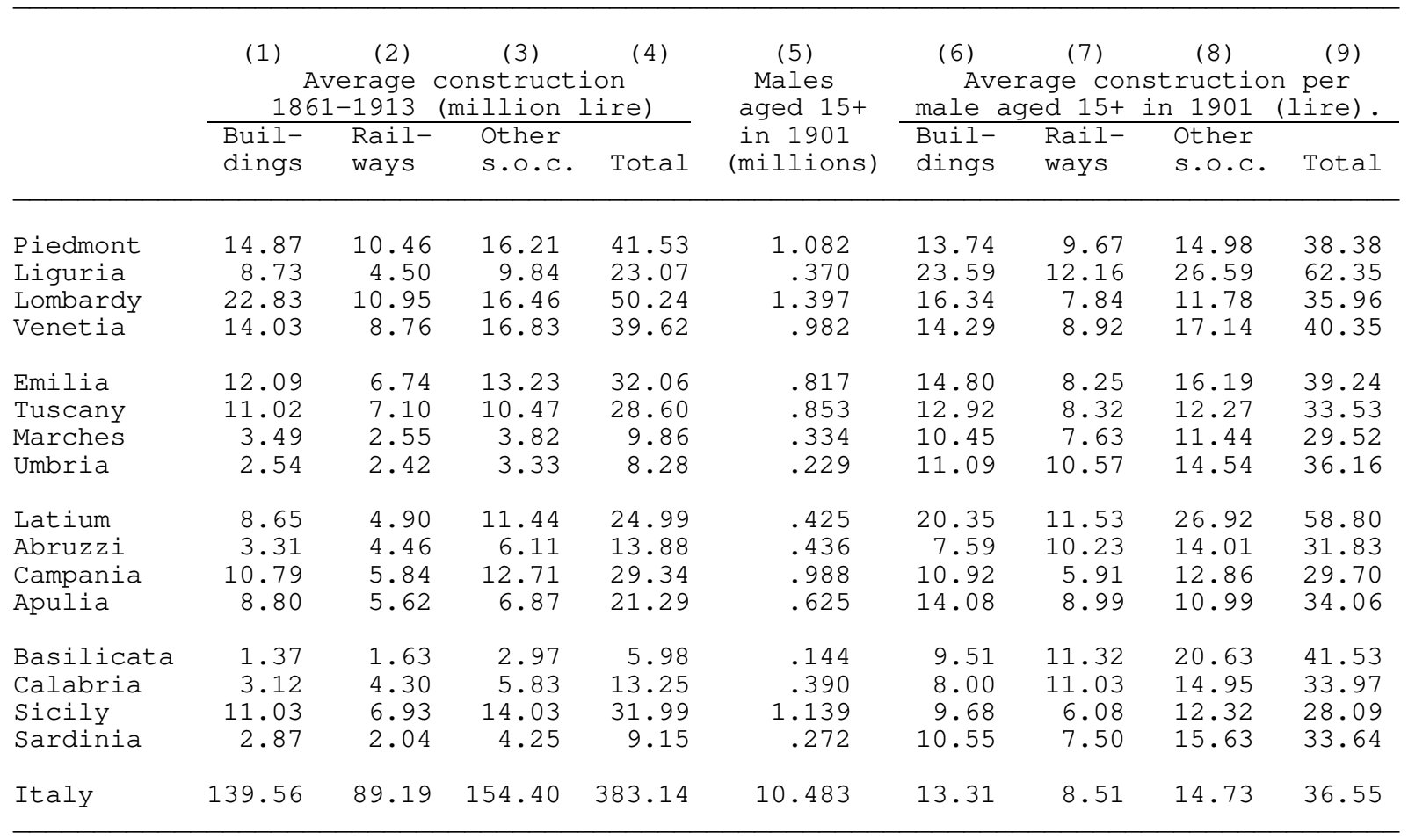

Source: see text. 
Map 1

Average regional construction per man, 1861-1913: ratios to national averages

Buildings

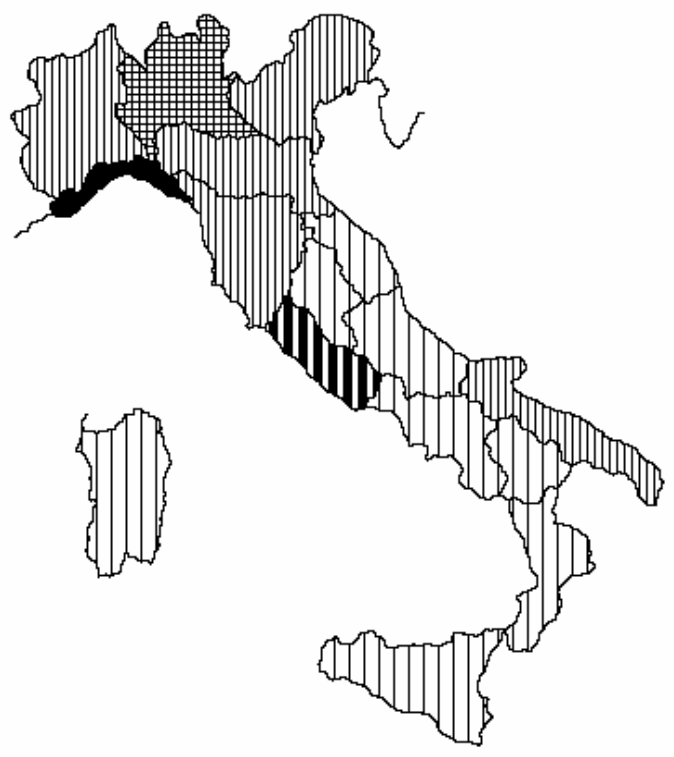

Other s.o.c.

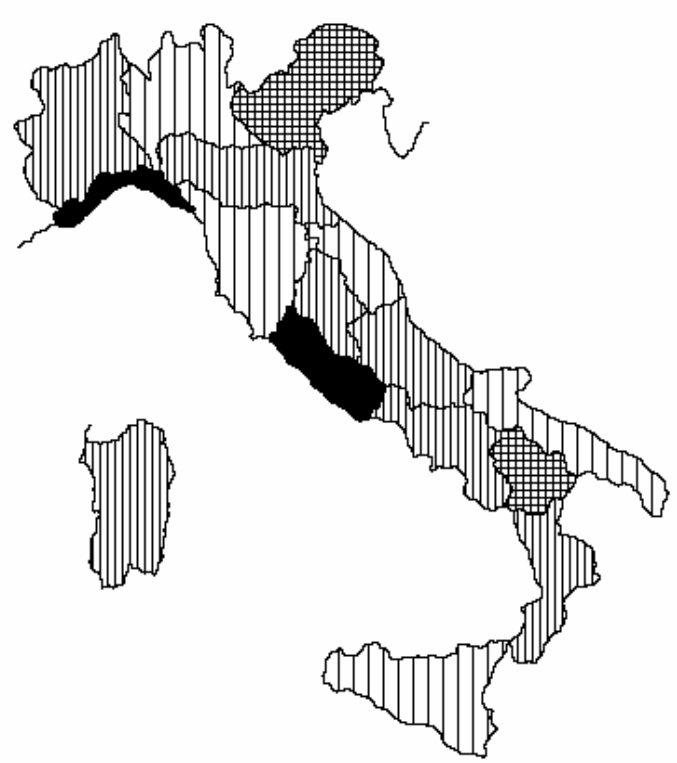

1.75 to 1.99

.85 to 1.14

(11in
|||||||| $\quad 1.45$ to 1.74

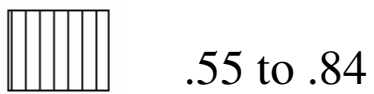

.55 to .84
Railways

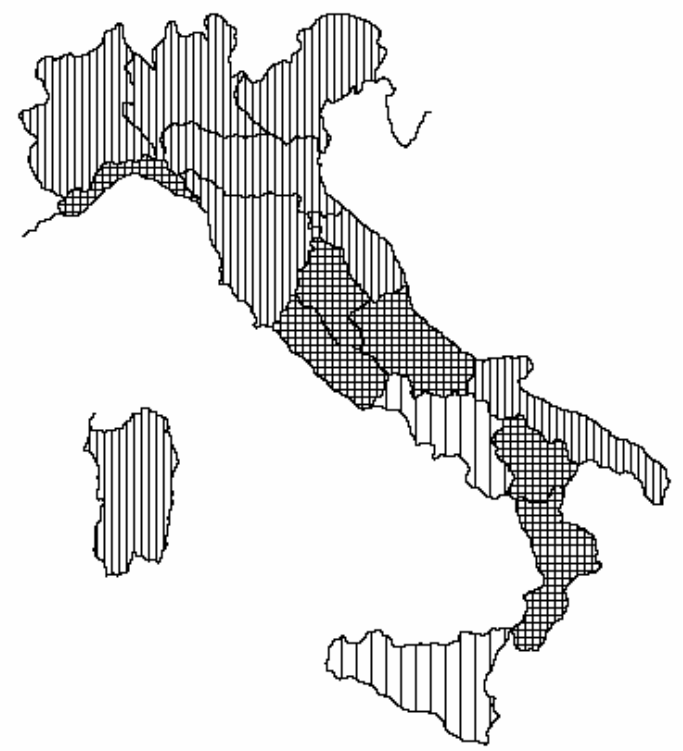

Total

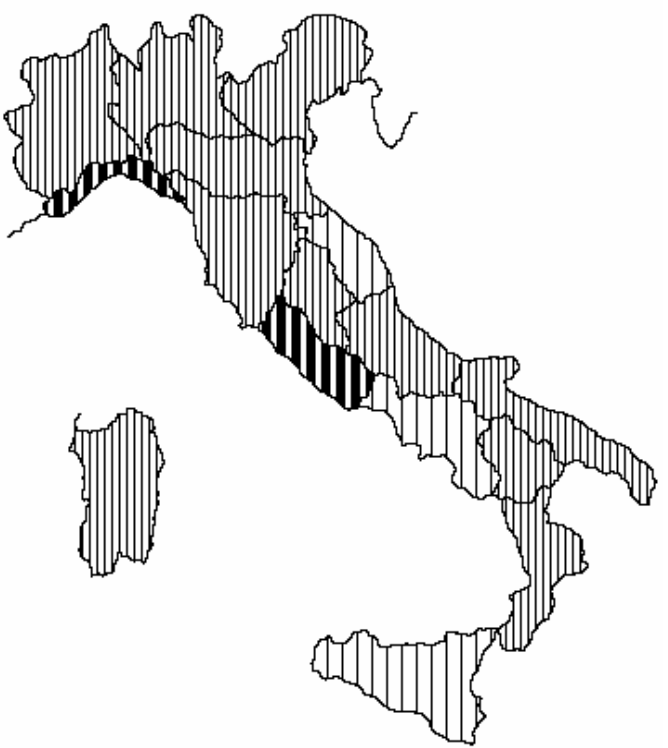

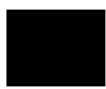

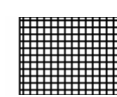

1.15 to 1.44
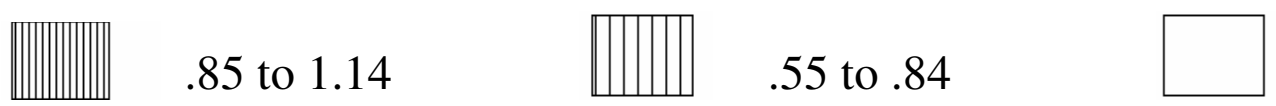

up to .54 
Figure 1

Average regional construction per man, 1861-1913: ratios to national averages
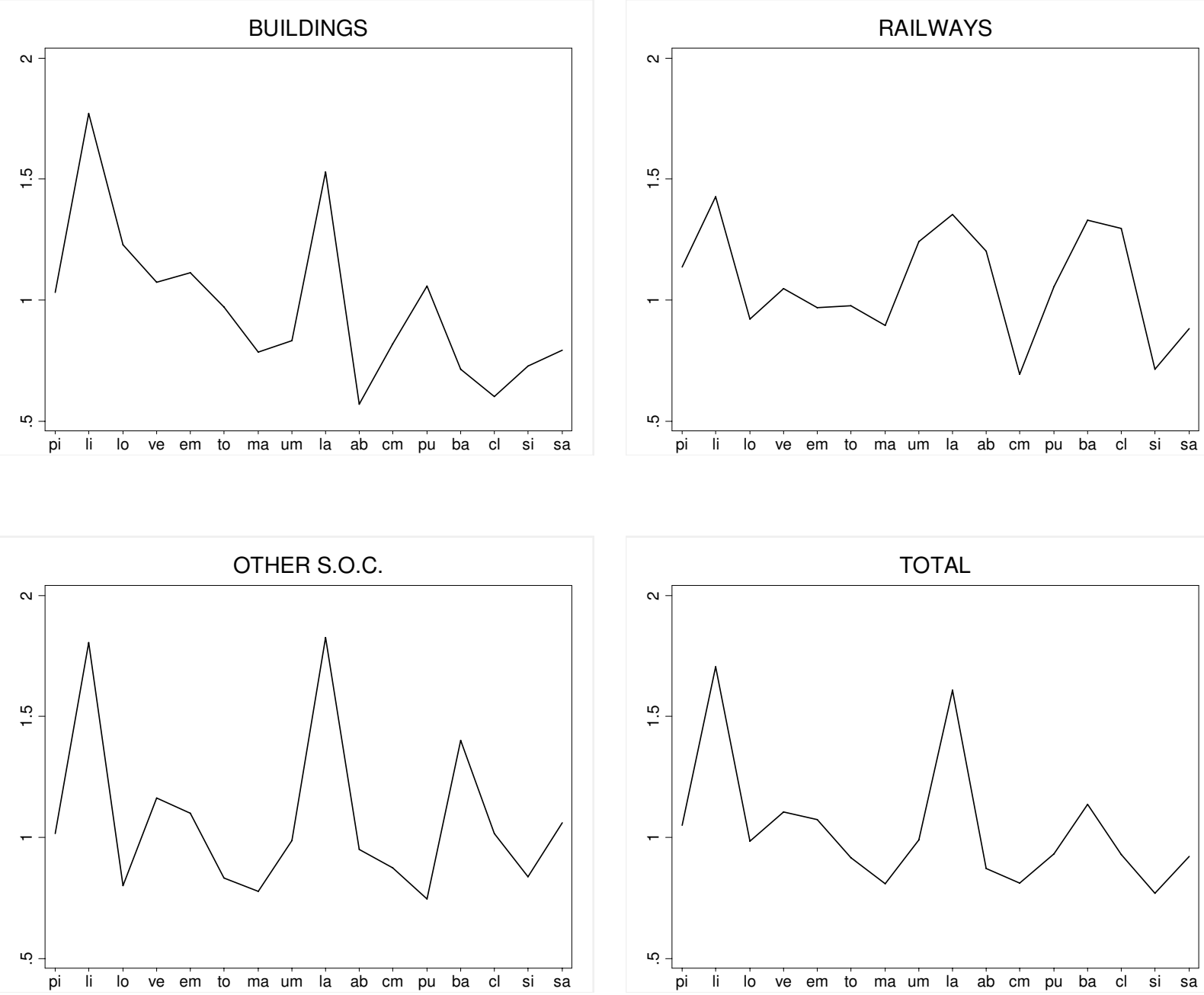

Order of regions: PI LI LO VE EM TO MA UM LA AB CM PU BA CL SI SA Source: Table 1. 
Figure 2

Construction value added per man: buildings (lire at 1911 prices)
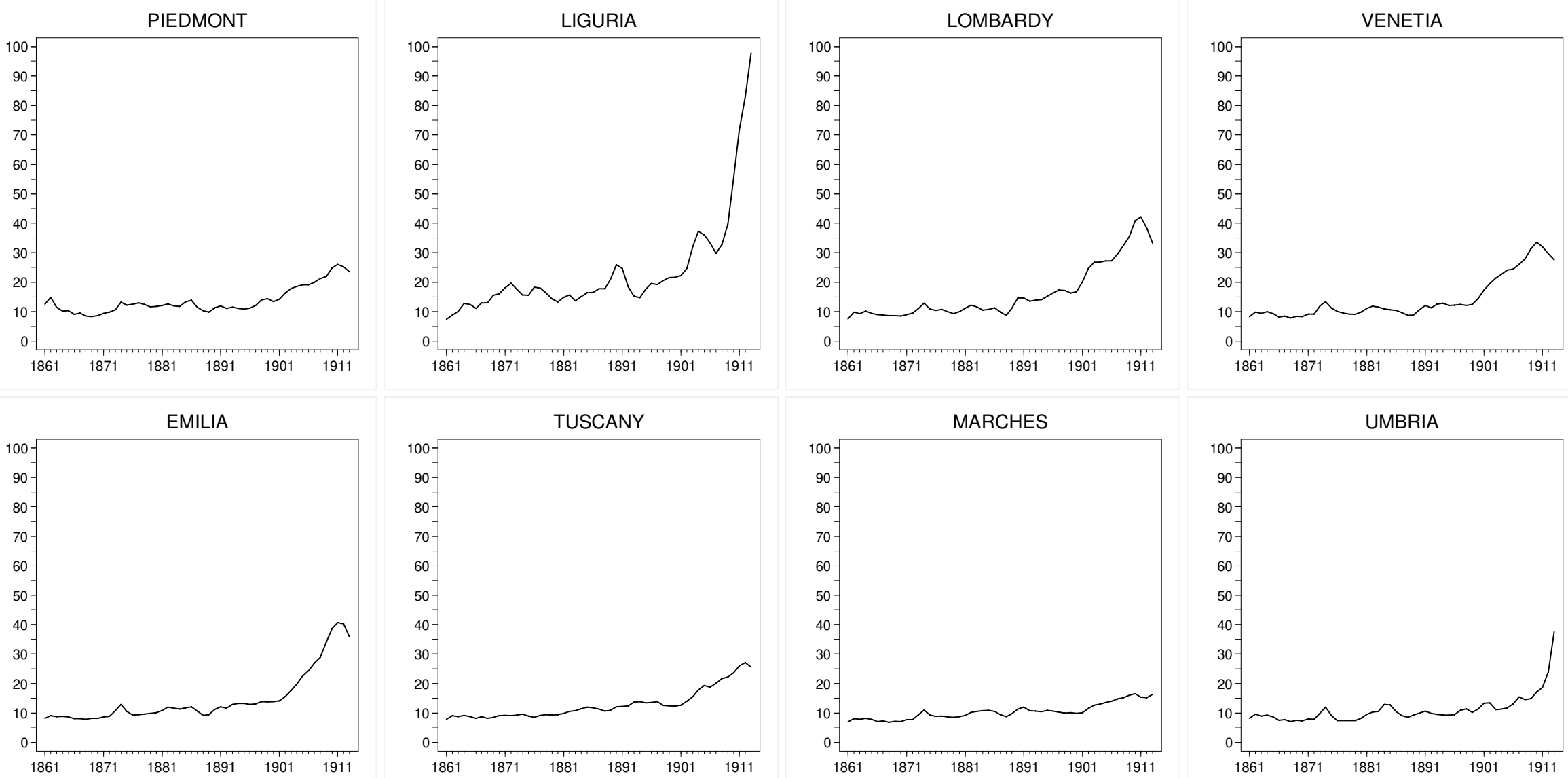
Figure 2, cont.
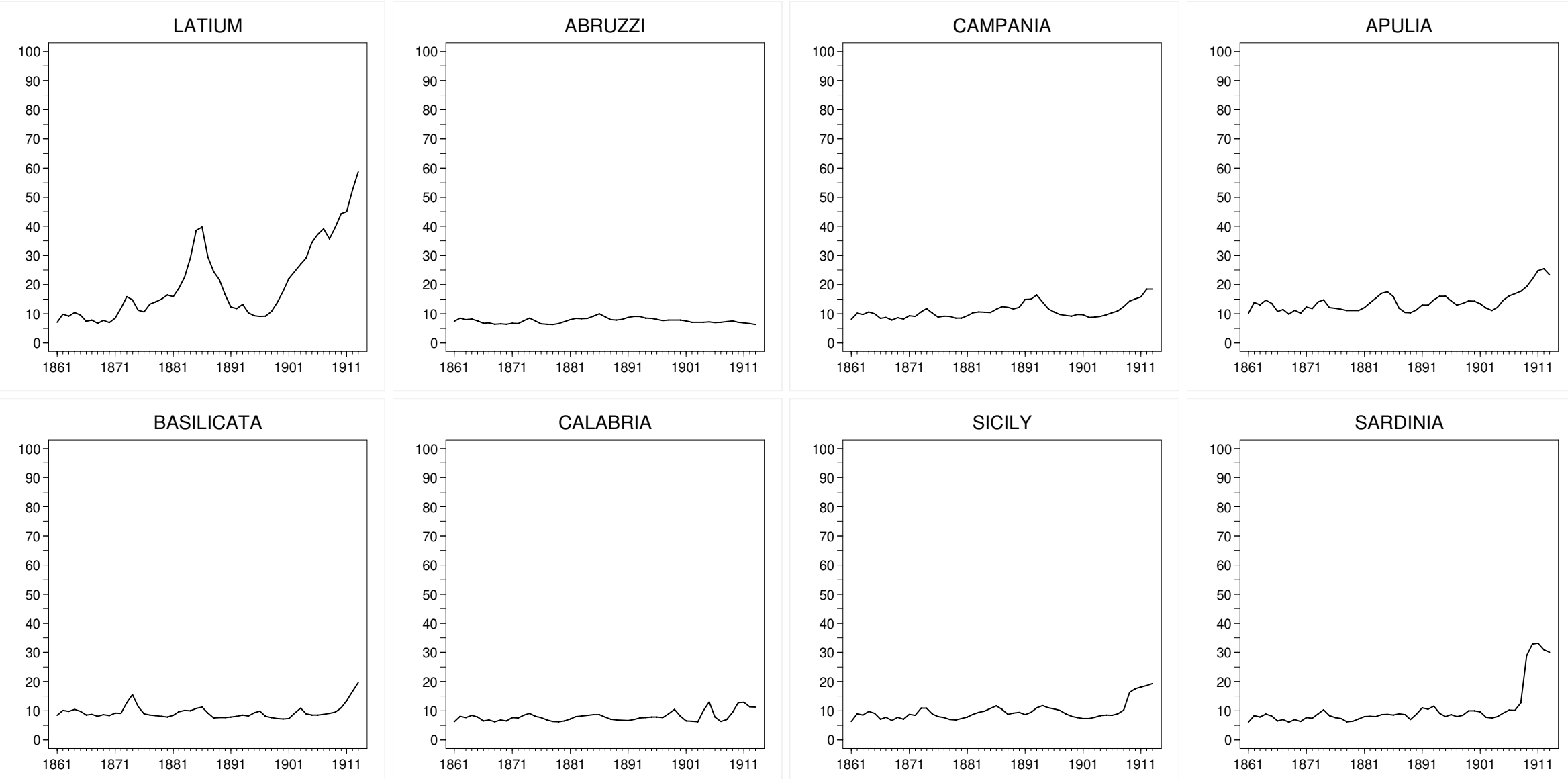

Source: see text. 
Figure 3

Construction value added per man: railways (lire at 1911 prices)
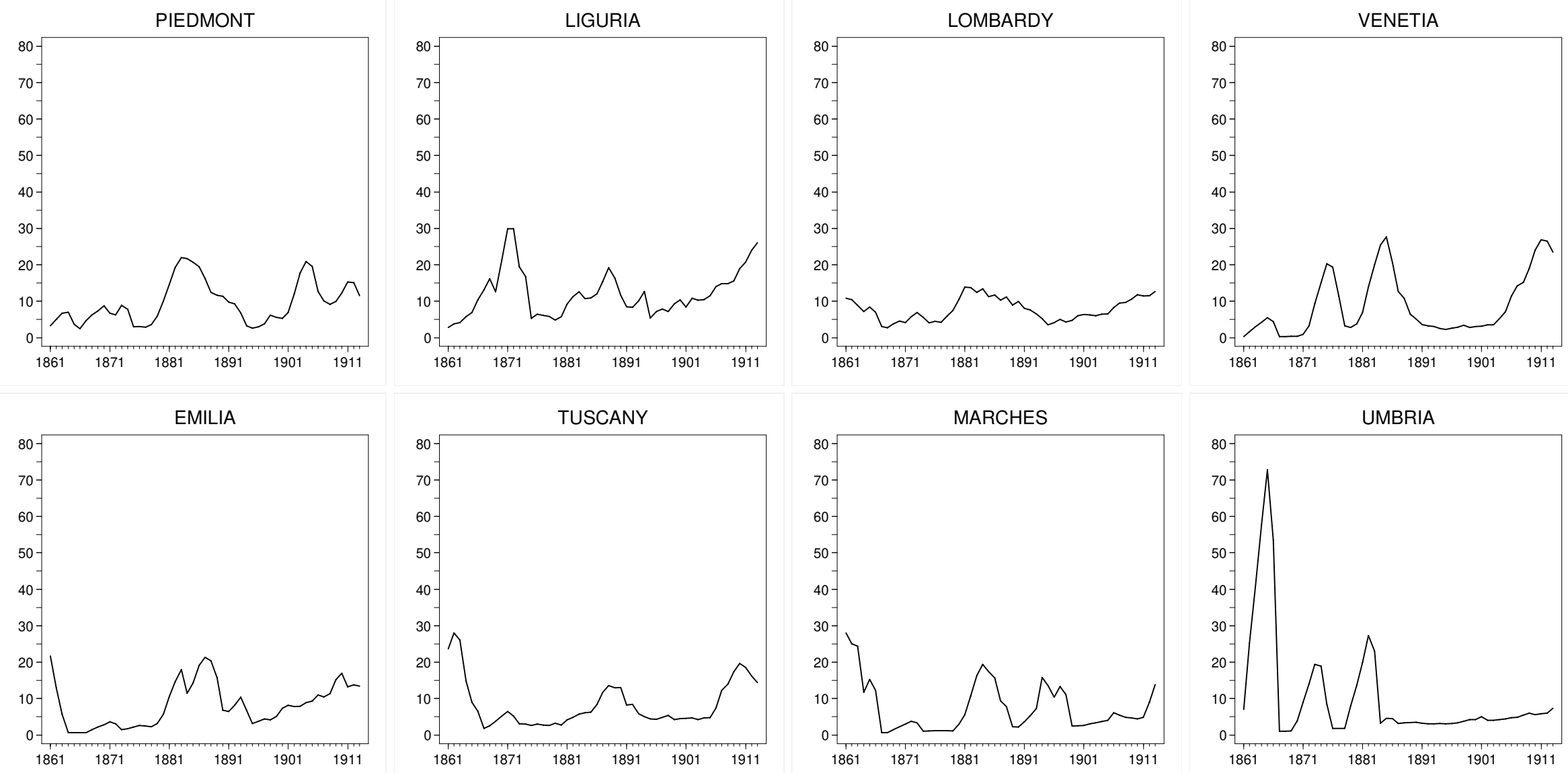
Figure 3, cont.
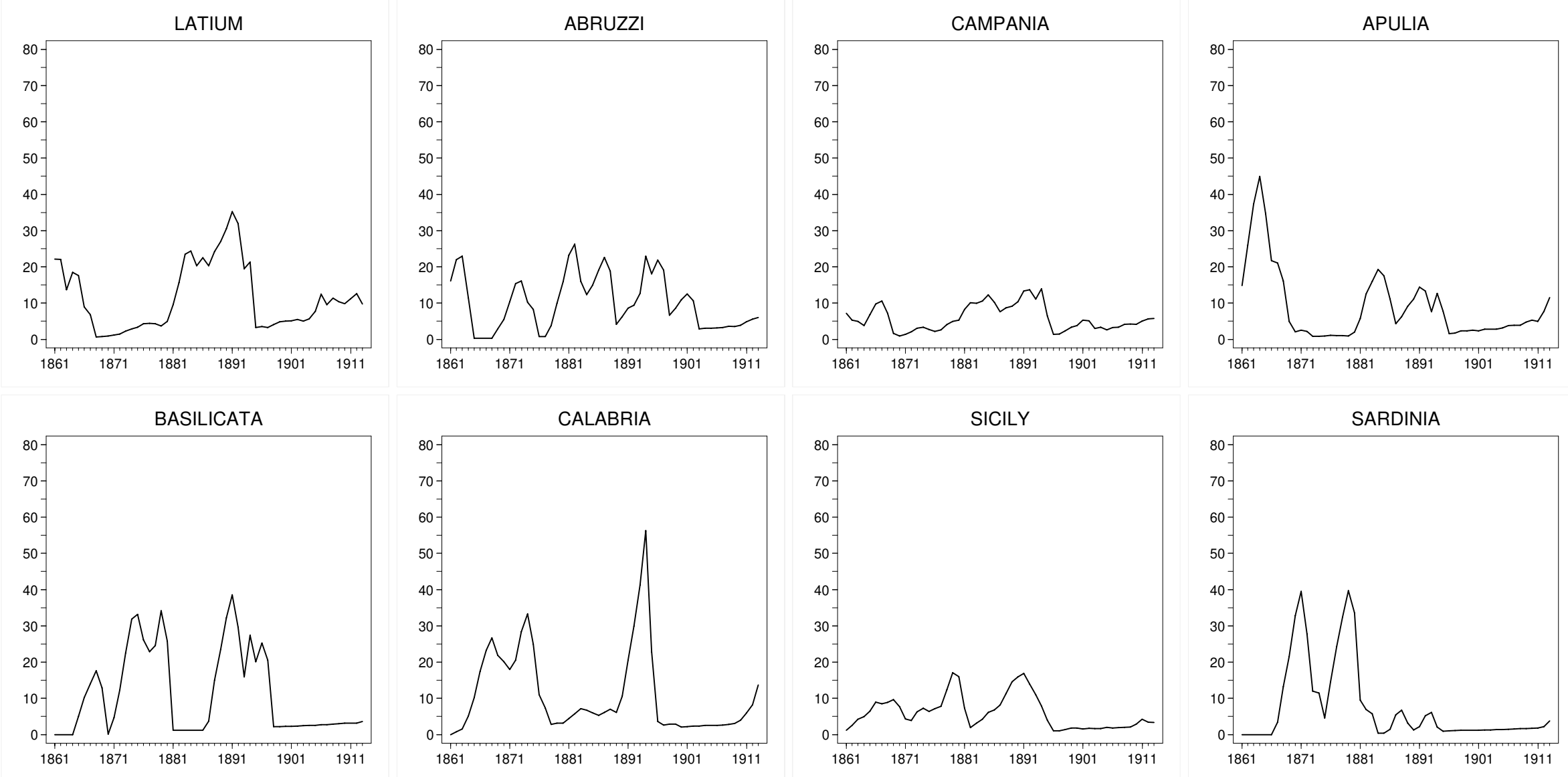

Source: see text. 
Figure 4

Construction value added per man: other social-overhead capital (lire at 1911 prices)
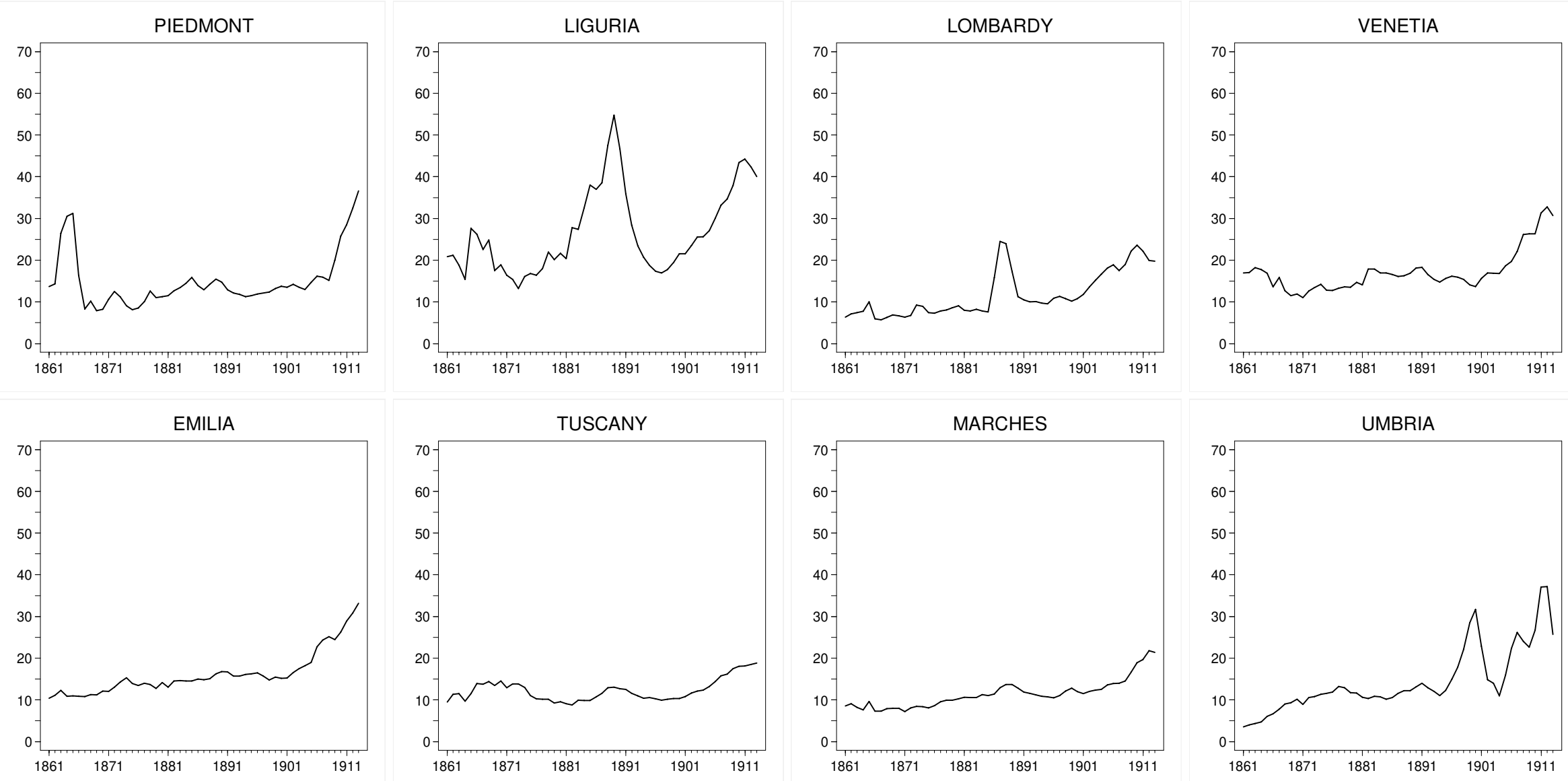
Figure 4, cont.

LATIUM
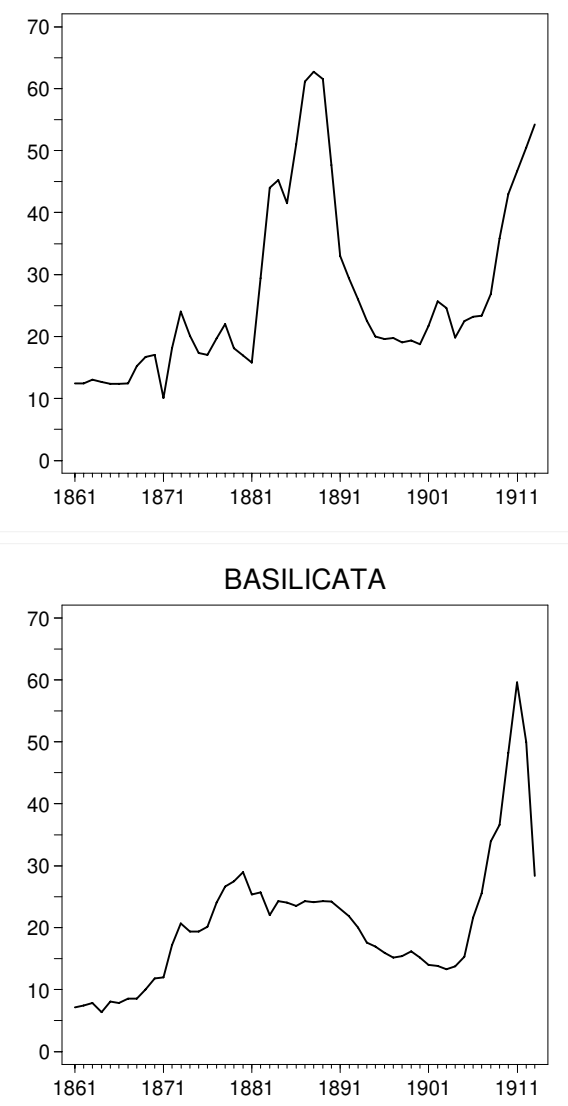

ABRUZZI

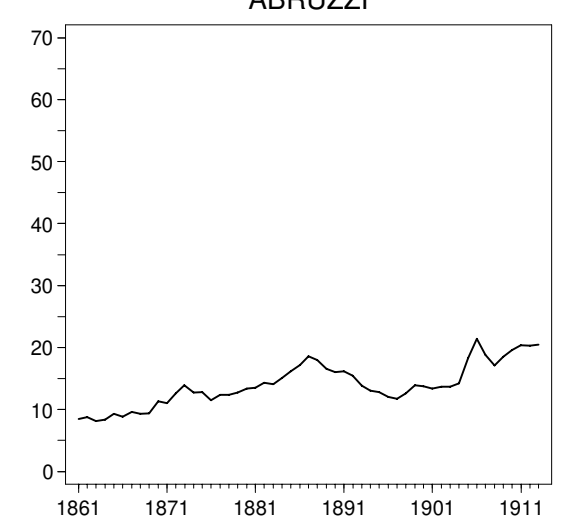

CALABRIA

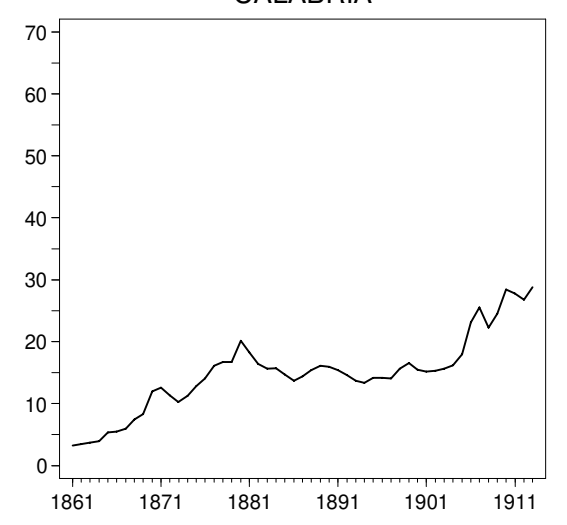

CAMPANIA

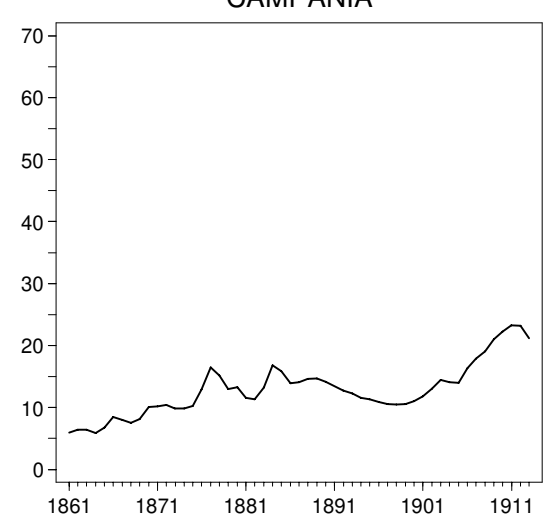

SICILY

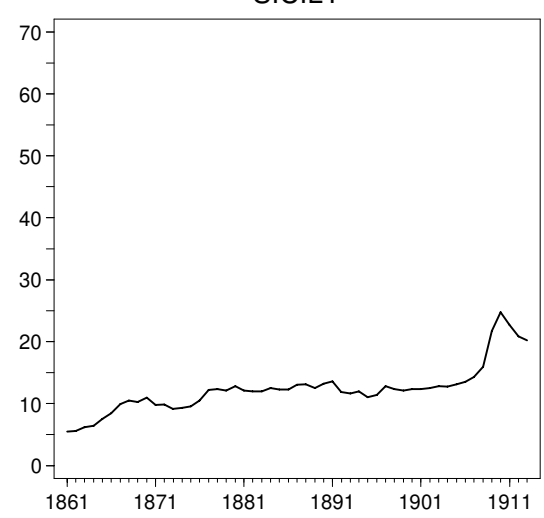

APULIA

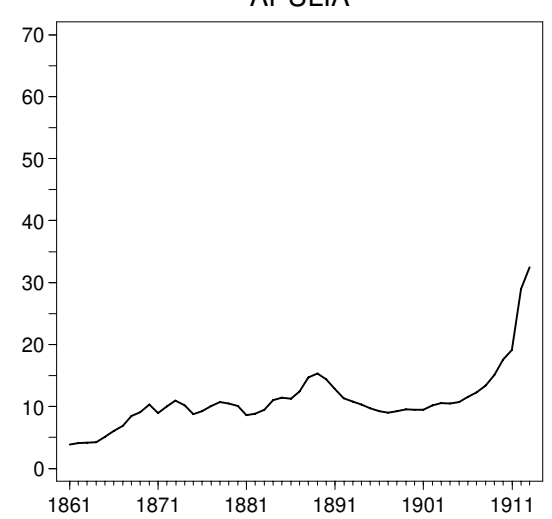

SARDINIA

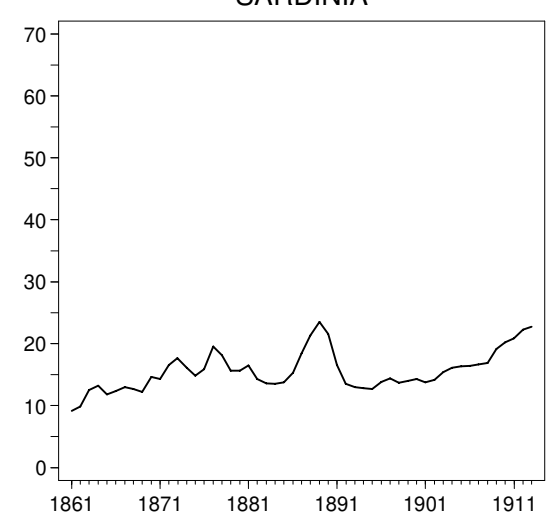

Source: see text. 
Figure 5

Construction value added per man: total (lire at 1911 prices)
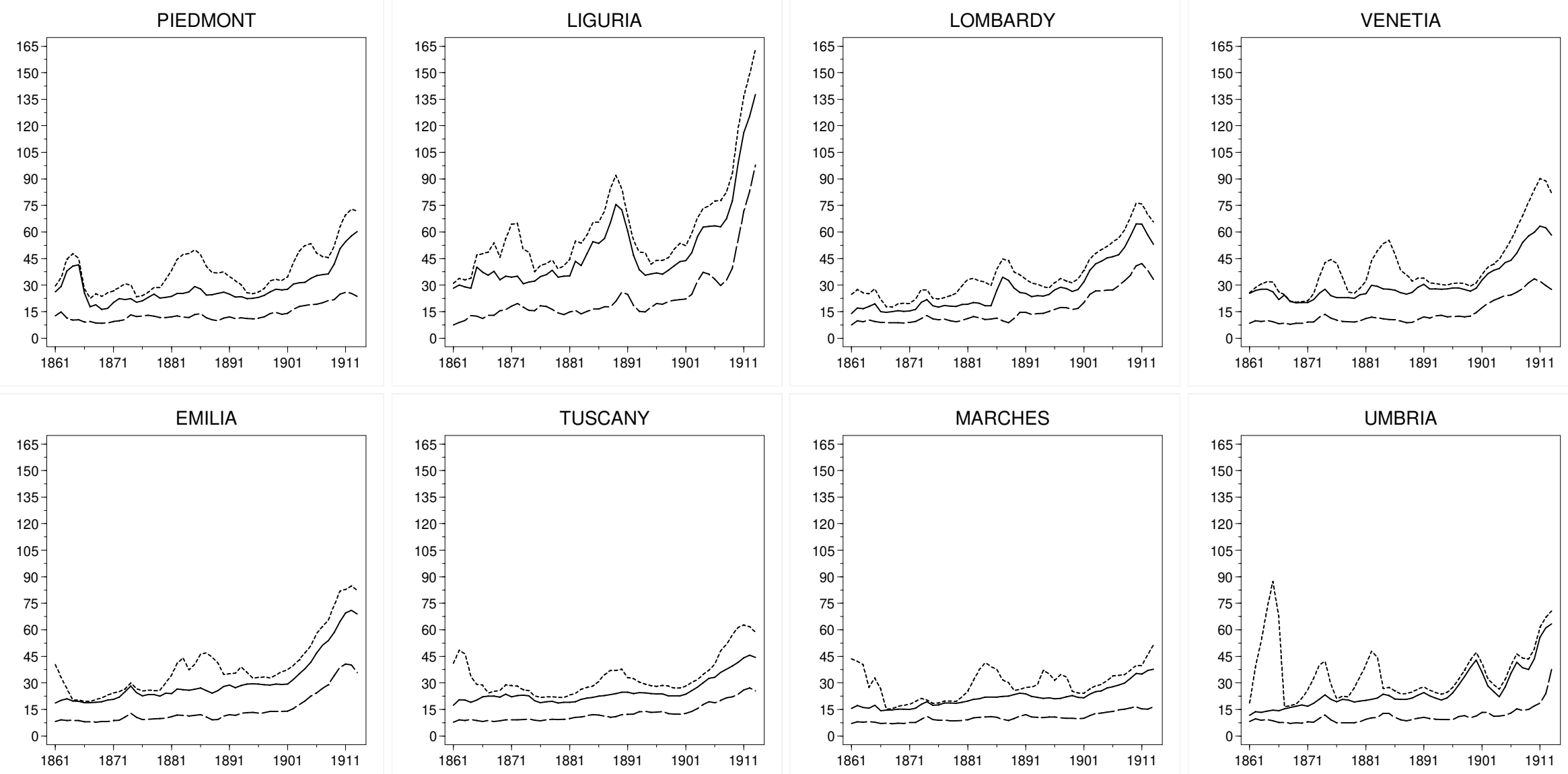


\section{Figure 5, cont.}
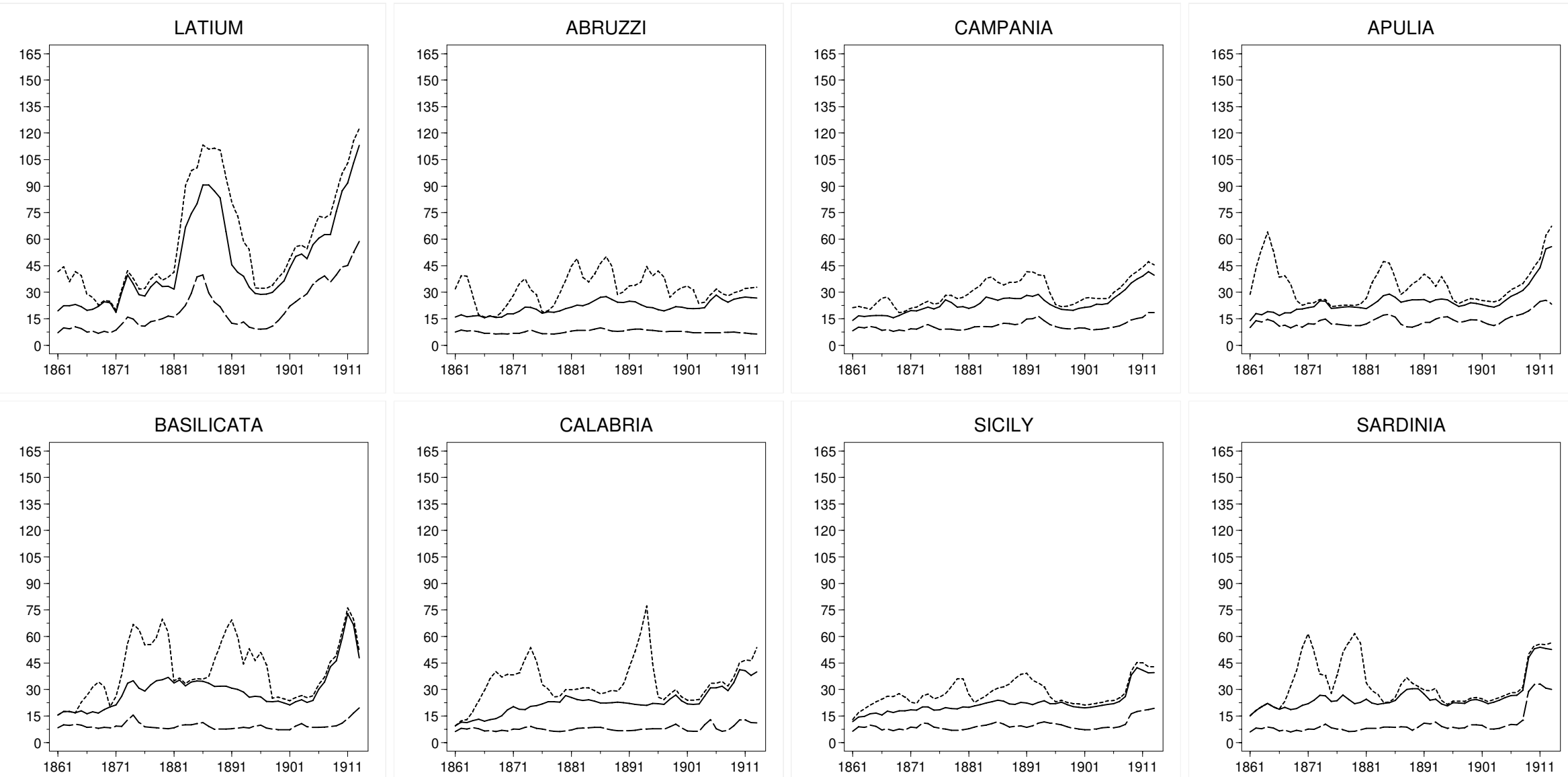

----- Total __ Total excluding railways $\quad \ldots---\quad$ Buildings

Source: see text. 
Figure 6

Construction by major area: buildings

A. Value added at 1911 prices (million lire)

B. Shares (percent)
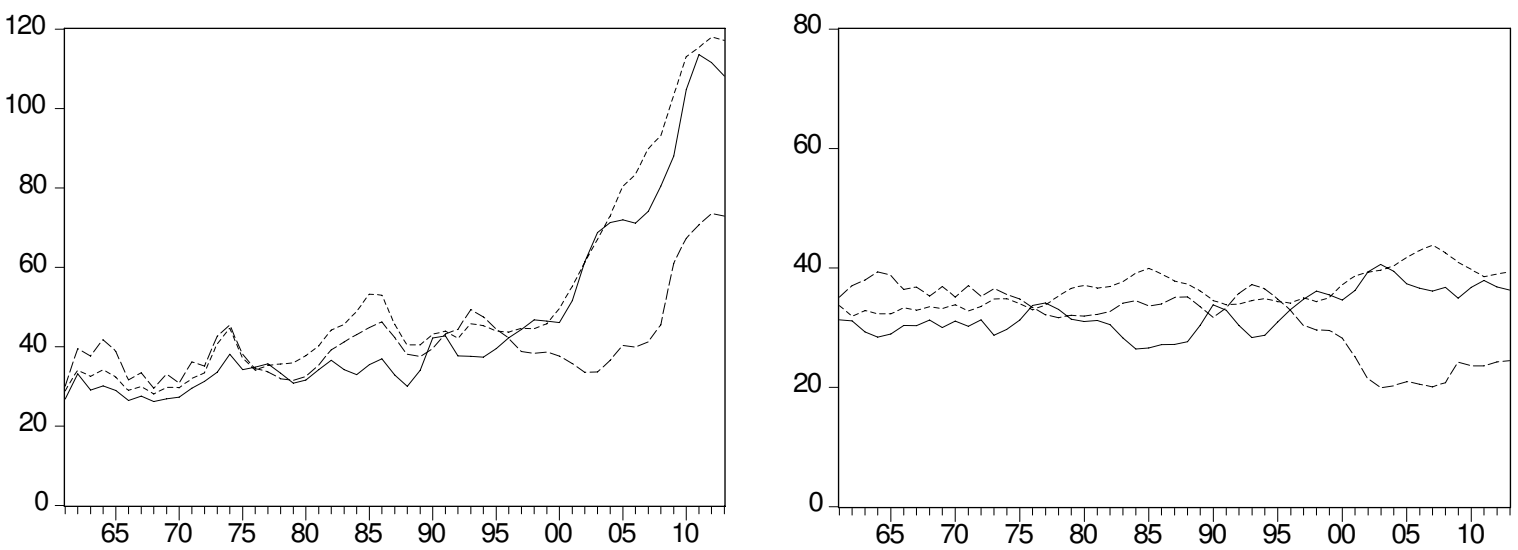

North-West

Center/North-East

South

Source: see text. 
Figure 7

Construction by major area: railways

A. Value added at 1911 prices (million lire)

B. Shares (percent)
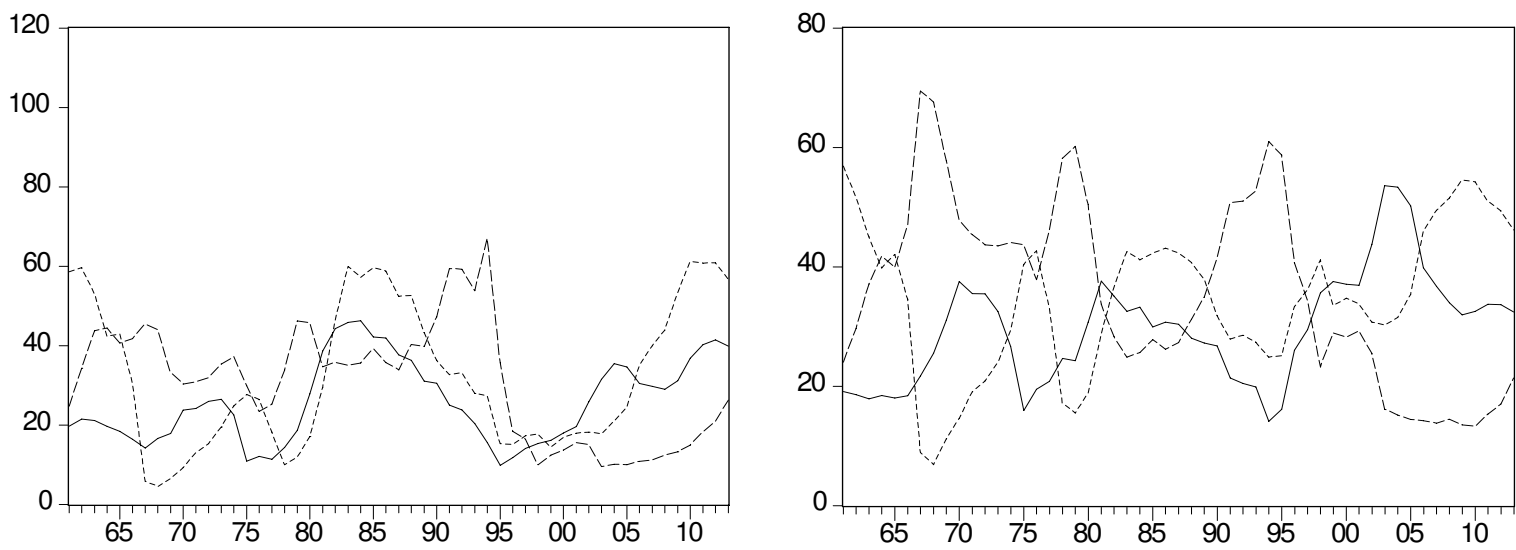

North-West

Center/North-East

South

Source: see text. 
Figure 8

Construction by major area: other social-overhead capital

A. Value added at 1911 prices (million lire)

B. Shares (percent)

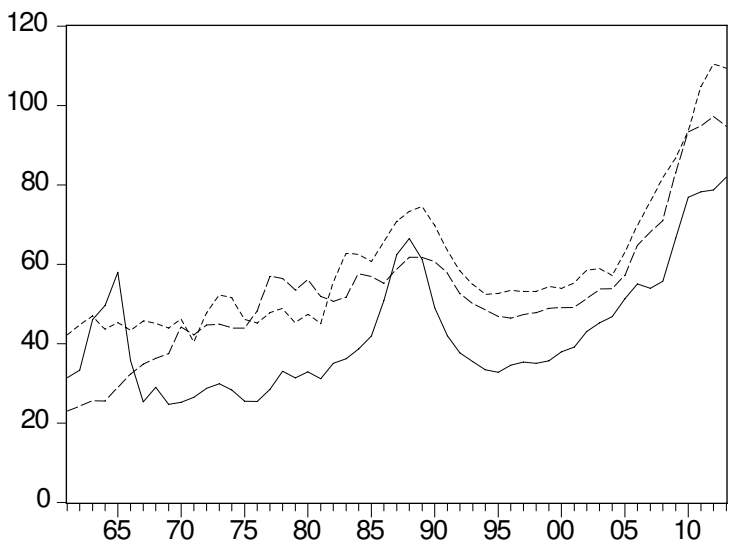

North-West

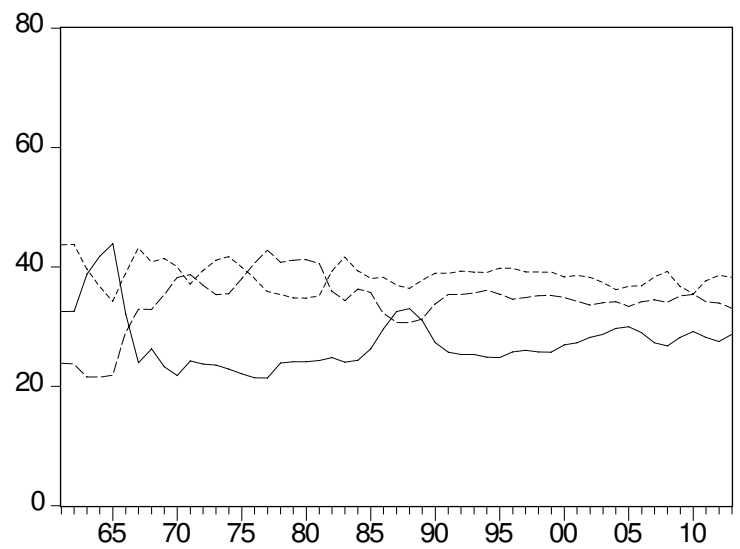

Center/North-East

South

Source: see text. 
Figure 9

Construction by major area: total

A. Value added at 1911 prices (million lire)

B. Shares (percent)

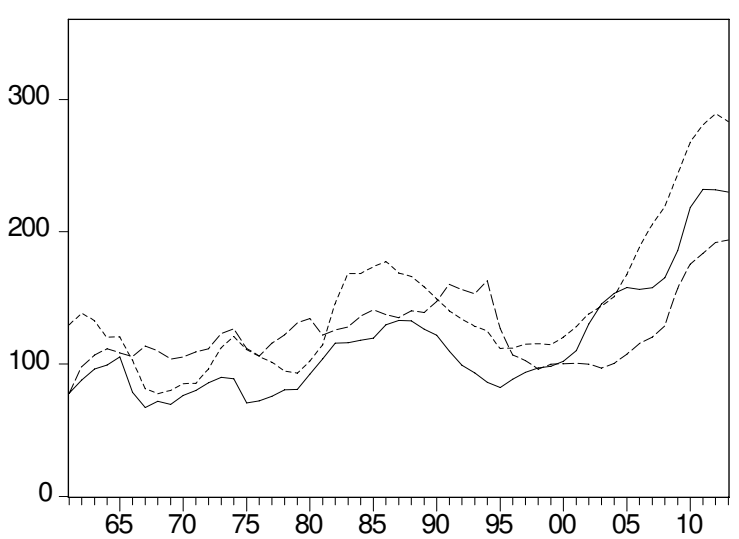

North-West

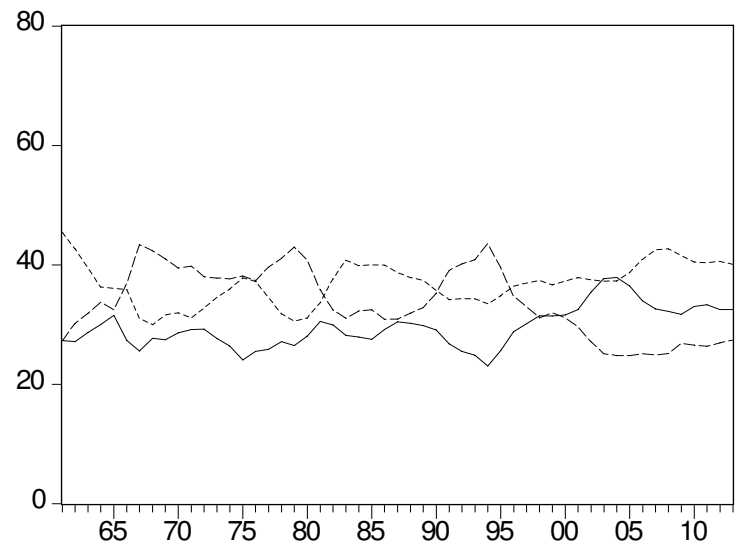

Center/North-East

South

Source: see text. 
Appendix A: New construction, 1861-1913: value added at 1911 prices (million lire)

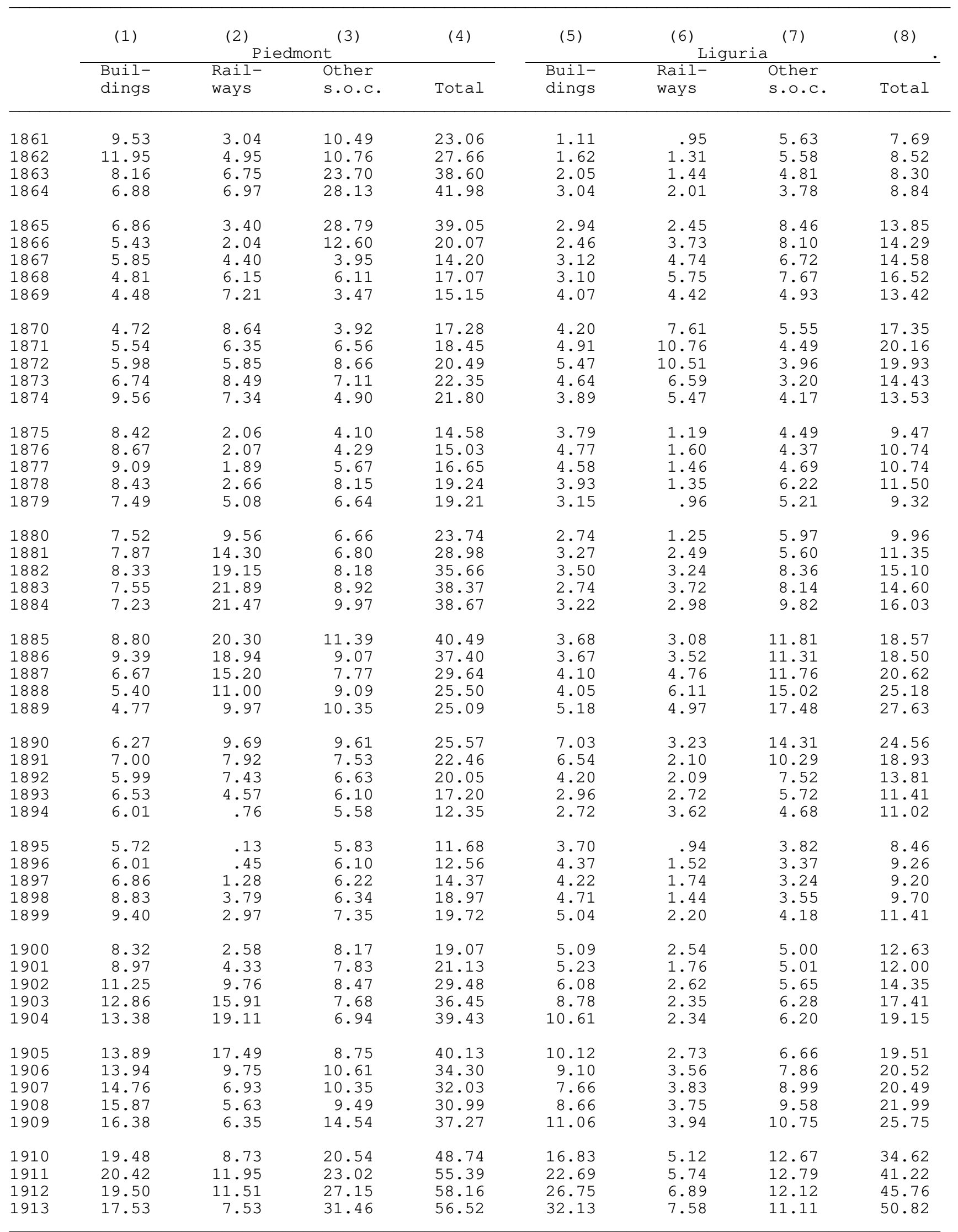




\begin{tabular}{|c|c|c|c|c|c|c|c|c|}
\hline & (9) & (10) & $d v^{(11)}$ & $(12)$ & (13) & (14) $\mathrm{Ve}$ & $(15)$ & (16) \\
\hline & Buil- & Rail- & Other & & Buil- & Rail- & Other & \\
\hline & dings & ways & s.o.c. & Total & dings & ways & S.O.C. & Total \\
\hline 1861 & 5.18 & 14.63 & 2.83 & 22.64 & 3.13 & .00 & 7.64 & 10.77 \\
\hline 1862 & 8.41 & 14.06 & 3.40 & 25.88 & 4.56 & 1.34 & 7.38 & 13.28 \\
\hline 1863 & 7.52 & 11.69 & 3.75 & 22.96 & 4.06 & 2.69 & 8.07 & 14.82 \\
\hline 1864 & 8.78 & 9.28 & 4.32 & 22.38 & 4.51 & 3.87 & 7.99 & 16.37 \\
\hline 1865 & 7.65 & 11.04 & 7.35 & 26.05 & 3.92 & 5.12 & 7.27 & 16.31 \\
\hline 1866 & 7.08 & 8.94 & 2.29 & 18.30 & 2.95 & 4.01 & 5.76 & 12.72 \\
\hline 1867 & 6.92 & 3.38 & 2.30 & 12.59 & 3.16 & .00 & 4.60 & 7.76 \\
\hline 1868 & 6.53 & 2.83 & 2.71 & 12.07 & 2.63 & .00 & 4.00 & 6.63 \\
\hline 1869 & 6.51 & 4.21 & 3.34 & 14.06 & 3.15 & .00 & 3.94 & 7.09 \\
\hline 1870 & 6.14 & 5.25 & 3.15 & 14.55 & 2.90 & .00 & 4.80 & 7.70 \\
\hline 1871 & 6.81 & 4.54 & 2.48 & 13.83 & 3.80 & .50 & 3.67 & 7.97 \\
\hline 1872 & 7.54 & 6.68 & 2.74 & 16.95 & 3.86 & 2.71 & 4.41 & 10.98 \\
\hline 1873 & 9.64 & 8.19 & 6.44 & 24.27 & 6.51 & 8.69 & 5.61 & 20.81 \\
\hline 1874 & 11.98 & 6.26 & 5.43 & 23.67 & 7.98 & 14.40 & 5.58 & 27.96 \\
\hline 1875 & 9.24 & 4.15 & 3.73 & 17.13 & 5.80 & 19.41 & 4.86 & 30.06 \\
\hline 1876 & 8.49 & 4.63 & 3.45 & 16.57 & 4.71 & 18.38 & 4.38 & 27.47 \\
\hline 1877 & 8.86 & 4.25 & 3.81 & 16.92 & 4.07 & 10.32 & 4.63 & 19.02 \\
\hline 1878 & 7.66 & 6.45 & 4.02 & 18.13 & 3.73 & 2.39 & 4.57 & 10.69 \\
\hline 1879 & 6.63 & 8.47 & 4.64 & 19.74 & 3.53 & 1.91 & 4.41 & 9.85 \\
\hline 1880 & 7.54 & 12.54 & 4.81 & 24.89 & 4.23 & 2.74 & 5.16 & 12.13 \\
\hline 1881 & 9.15 & 17.02 & 3.34 & 29.51 & 5.47 & 5.74 & 5.16 & 16.36 \\
\hline 1882 & 10.56 & 16.60 & 3.27 & 30.43 & 6.25 & 12.63 & 5.54 & 24.42 \\
\hline 1883 & 9.61 & 14.68 & 3.16 & 27.45 & 5.84 & 18.55 & 6.77 & 31.17 \\
\hline 1884 & 7.96 & 15.82 & 3.72 & 27.50 & 5.29 & 23.85 & 7.35 & 36.49 \\
\hline 1885 & 8.18 & 12.56 & 3.51 & 24.25 & 4.89 & 25.92 & 7.64 & 38.45 \\
\hline 1886 & 8.80 & 13.05 & 14.31 & 36.17 & 4.66 & 18.69 & 6.47 & 29.82 \\
\hline 1887 & 6.76 & 10.83 & 26.40 & 43.98 & 3.77 & 10.93 & 6.02 & 20.72 \\
\hline 1888 & 5.01 & 11.74 & 25.71 & 42.46 & 2.87 & 8.95 & 6.13 & 17.95 \\
\hline 1889 & 8.41 & 8.48 & 15.80 & 32.69 & 3.03 & 4.53 & 6.33 & 13.90 \\
\hline 1890 & 13.15 & 9.85 & 7.47 & 30.48 & 4.75 & 3.26 & 7.84 & 15.85 \\
\hline 1891 & 13.27 & 7.32 & 6.59 & 27.17 & 6.14 & 1.76 & 8.18 & 16.08 \\
\hline 1892 & 11.61 & 6.63 & 5.66 & 23.90 & 5.37 & 1.50 & 6.27 & 13.14 \\
\hline 1893 & 12.06 & 5.04 & 5.58 & 22.68 & 6.61 & 1.23 & 5.01 & 12.85 \\
\hline 1894 & 12.32 & 3.10 & 5.46 & 20.87 & 6.97 & .71 & 4.86 & 12.54 \\
\hline 1895 & 13.63 & .79 & 5.10 & 19.52 & 6.15 & .54 & 5.59 & 12.29 \\
\hline 1896 & 15.15 & 1.54 & 6.39 & 23.08 & 6.16 & .81 & 5.71 & 12.68 \\
\hline 1897 & 16.34 & 2.53 & 6.93 & 25.80 & 6.37 & 1.01 & 5.38 & 12.76 \\
\hline 1898 & 16.10 & 1.42 & 5.66 & 23.18 & 5.93 & 1.51 & 4.63 & 12.07 \\
\hline 1899 & 14.76 & 1.92 & 4.74 & 21.42 & 6.34 & .78 & 3.61 & 10.73 \\
\hline 1900 & 15.32 & 3.64 & 6.00 & 24.97 & 8.30 & 1.05 & 3.92 & 13.27 \\
\hline 1901 & 19.72 & 4.03 & 7.26 & 31.01 & 11.01 & 1.05 & 5.81 & 17.87 \\
\hline 1902 & 26.03 & 3.69 & 9.29 & 39.01 & 13.18 & 1.42 & 6.74 & 21.34 \\
\hline 1903 & 28.78 & 3.10 & 11.36 & 43.24 & 14.94 & 1.33 & 6.66 & 22.93 \\
\hline 1904 & 28.61 & 3.46 & 13.22 & 45.30 & 16.12 & 2.86 & 6.54 & 25.52 \\
\hline 1905 & 28.95 & 3.52 & 15.04 & 47.51 & 17.35 & 4.82 & 8.27 & 30.44 \\
\hline 1906 & 28.76 & 5.50 & 16.33 & 50.59 & 17.62 & 8.90 & 9.71 & 36.23 \\
\hline 1907 & 31.80 & 7.09 & 14.26 & 53.15 & 18.99 & 11.50 & 12.18 & 42.67 \\
\hline 1908 & 35.45 & 7.07 & 16.36 & 58.88 & 20.78 & 12.27 & 16.13 & 49.19 \\
\hline 1909 & 39.38 & 7.92 & 20.66 & 67.96 & 23.95 & 15.86 & 16.08 & 55.90 \\
\hline 1910 & 46.62 & 9.39 & 22.33 & 78.35 & 25.94 & 20.58 & 15.67 & 62.19 \\
\hline 1911 & 47.77 & 8.56 & 19.74 & 76.07 & 24.16 & 23.21 & 19.87 & 67.25 \\
\hline 1912 & 42.05 & 8.54 & 16.47 & 67.07 & 21.65 & 22.64 & 21.27 & 65.55 \\
\hline 1913 & 34.62 & 9.87 & 16.13 & 60.62 & 19.39 & 19.49 & 19.17 & 58.04 \\
\hline
\end{tabular}




\begin{tabular}{|c|c|c|c|c|c|c|c|c|}
\hline & $(17)$ & (18) & Emilia & $(20)$ & \multicolumn{4}{|c|}{ Tuscany } \\
\hline & $\begin{array}{l}\text { Buil- } \\
\text { dings }\end{array}$ & $\begin{array}{l}\text { Rail- } \\
\text { ways }\end{array}$ & $\begin{array}{l}\text { Other } \\
\text { s.o.c. }\end{array}$ & Total & $\begin{array}{l}\text { Buil- } \\
\text { dings }\end{array}$ & $\begin{array}{l}\text { Rail- } \\
\text { ways }\end{array}$ & $\begin{array}{l}\text { Other } \\
\text { s.o.c. }\end{array}$ & Total \\
\hline $\begin{array}{l}1861 \\
1862 \\
1863 \\
1864\end{array}$ & $\begin{array}{l}2.01 \\
2.66 \\
2.37 \\
2.42\end{array}$ & $\begin{array}{r}17.39 \\
10.14 \\
4.09 \\
.00\end{array}$ & $\begin{array}{l}2.59 \\
2.70 \\
3.81 \\
2.64\end{array}$ & $\begin{array}{r}21.99 \\
15.49 \\
10.27 \\
5.06\end{array}$ & $\begin{array}{l}1.85 \\
2.81 \\
2.50 \\
2.83\end{array}$ & $\begin{array}{l}19.84 \\
23.52 \\
21.70 \\
12.00\end{array}$ & $\begin{array}{l}3.58 \\
4.71 \\
4.58 \\
3.42\end{array}$ & $\begin{array}{l}25.27 \\
31.04 \\
28.79 \\
18.25\end{array}$ \\
\hline $\begin{array}{l}1865 \\
1866 \\
1867 \\
1868 \\
1869\end{array}$ & $\begin{array}{l}2.27 \\
1.88 \\
1.85 \\
1.75 \\
2.00\end{array}$ & $\begin{array}{r}.00 \\
.00 \\
.00 \\
.61 \\
1.16\end{array}$ & $\begin{array}{l}2.75 \\
2.21 \\
2.38 \\
2.62 \\
2.49\end{array}$ & $\begin{array}{l}5.02 \\
4.09 \\
4.23 \\
4.98 \\
5.64\end{array}$ & $\begin{array}{l}2.47 \\
2.14 \\
2.55 \\
2.15 \\
2.47\end{array}$ & $\begin{array}{r}6.92 \\
4.81 \\
.77 \\
1.35 \\
2.32\end{array}$ & $\begin{array}{l}5.11 \\
6.84 \\
6.97 \\
7.20 \\
6.18\end{array}$ & $\begin{array}{l}14.50 \\
13.79 \\
10.28 \\
10.70 \\
10.97\end{array}$ \\
\hline $\begin{array}{l}1870 \\
1871 \\
1872 \\
1873 \\
1874\end{array}$ & $\begin{array}{l}1.96 \\
2.37 \\
2.56 \\
4.09 \\
5.81\end{array}$ & $\begin{array}{r}1.68 \\
2.25 \\
1.75 \\
.34 \\
.59\end{array}$ & $\begin{array}{l}3.50 \\
3.28 \\
4.09 \\
5.02 \\
5.26\end{array}$ & $\begin{array}{r}7.14 \\
7.89 \\
8.39 \\
9.46 \\
11.66\end{array}$ & $\begin{array}{l}2.84 \\
2.92 \\
2.90 \\
2.98 \\
3.33\end{array}$ & $\begin{array}{l}3.53 \\
4.45 \\
3.32 \\
1.35 \\
1.37\end{array}$ & $\begin{array}{l}6.98 \\
5.61 \\
6.45 \\
6.66 \\
5.71\end{array}$ & $\begin{array}{l}13.34 \\
12.98 \\
12.67 \\
10.99 \\
10.42\end{array}$ \\
\hline $\begin{array}{l}1875 \\
1876 \\
1877 \\
1878 \\
1879\end{array}$ & $\begin{array}{l}3.92 \\
2.86 \\
2.96 \\
3.13 \\
3.24\end{array}$ & $\begin{array}{r}.96 \\
1.30 \\
1.13 \\
1.01 \\
1.70\end{array}$ & $\begin{array}{l}4.55 \\
4.21 \\
4.27 \\
4.09 \\
3.77\end{array}$ & $\begin{array}{l}9.43 \\
8.37 \\
8.36 \\
8.23 \\
8.71\end{array}$ & $\begin{array}{l}2.69 \\
2.40 \\
2.90 \\
3.13 \\
3.05\end{array}$ & $\begin{array}{r}.98 \\
1.25 \\
1.04 \\
.93 \\
1.41\end{array}$ & $\begin{array}{l}4.41 \\
3.86 \\
3.69 \\
3.67 \\
3.13\end{array}$ & $\begin{array}{l}8.08 \\
7.51 \\
7.63 \\
7.73 \\
7.60\end{array}$ \\
\hline $\begin{array}{l}1880 \\
1881 \\
1882 \\
1883 \\
1884\end{array}$ & $\begin{array}{l}3.44 \\
4.06 \\
4.89 \\
4.64 \\
4.34\end{array}$ & $\begin{array}{r}3.77 \\
7.65 \\
10.93 \\
13.30 \\
7.84\end{array}$ & $\begin{array}{l}4.51 \\
4.14 \\
4.59 \\
4.96 \\
4.97\end{array}$ & $\begin{array}{l}11.71 \\
15.85 \\
20.40 \\
22.90 \\
17.15\end{array}$ & $\begin{array}{l}3.11 \\
3.47 \\
4.05 \\
4.21 \\
4.76\end{array}$ & $\begin{array}{r}.91 \\
2.05 \\
2.58 \\
3.32 \\
3.65\end{array}$ & $\begin{array}{l}3.30 \\
3.11 \\
2.97 \\
3.85 \\
3.97\end{array}$ & $\begin{array}{r}7.32 \\
8.63 \\
9.60 \\
11.38 \\
12.38\end{array}$ \\
\hline $\begin{array}{l}1885 \\
1886 \\
1887 \\
1888 \\
1889\end{array}$ & $\begin{array}{l}4.61 \\
4.81 \\
3.66 \\
2.37 \\
2.52\end{array}$ & $\begin{array}{l}10.08 \\
14.06 \\
15.59 \\
14.58 \\
10.49\end{array}$ & $\begin{array}{l}4.95 \\
4.91 \\
4.64 \\
4.47 \\
5.07\end{array}$ & $\begin{array}{l}19.64 \\
23.78 \\
23.89 \\
21.43 \\
18.08\end{array}$ & $\begin{array}{l}5.15 \\
4.92 \\
4.51 \\
3.84 \\
4.07\end{array}$ & $\begin{array}{l}3.78 \\
5.52 \\
8.35 \\
9.75 \\
9.24\end{array}$ & $\begin{array}{l}3.93 \\
4.29 \\
4.92 \\
5.90 \\
5.84\end{array}$ & $\begin{array}{l}12.86 \\
14.72 \\
17.78 \\
19.49 \\
19.15\end{array}$ \\
\hline $\begin{array}{l}1890 \\
1891 \\
1892 \\
1893 \\
1894\end{array}$ & $\begin{array}{l}4.00 \\
4.71 \\
4.36 \\
5.40 \\
5.61\end{array}$ & $\begin{array}{l}3.20 \\
2.91 \\
4.33 \\
6.04 \\
3.00\end{array}$ & $\begin{array}{l}5.36 \\
5.14 \\
4.68 \\
4.81 \\
5.20\end{array}$ & $\begin{array}{l}12.56 \\
12.76 \\
13.37 \\
16.25 \\
13.81\end{array}$ & $\begin{array}{l}5.09 \\
5.18 \\
5.35 \\
6.33 \\
6.54\end{array}$ & $\begin{array}{l}9.19 \\
5.07 \\
5.29 \\
2.98 \\
2.28\end{array}$ & $\begin{array}{l}5.46 \\
5.31 \\
4.50 \\
3.83 \\
3.47\end{array}$ & $\begin{array}{l}19.74 \\
15.56 \\
15.14 \\
13.14 \\
12.29\end{array}$ \\
\hline $\begin{array}{l}1895 \\
1896 \\
1897 \\
1898 \\
1899\end{array}$ & $\begin{array}{l}5.64 \\
5.34 \\
5.46 \\
6.03 \\
5.96\end{array}$ & $\begin{array}{r}.09 \\
.48 \\
.96 \\
.67 \\
1.39\end{array}$ & $\begin{array}{l}5.35 \\
5.15 \\
3.99 \\
3.51 \\
4.28\end{array}$ & $\begin{array}{l}11.08 \\
10.98 \\
10.41 \\
10.21 \\
11.63\end{array}$ & $\begin{array}{l}6.11 \\
6.13 \\
6.38 \\
5.22 \\
5.11\end{array}$ & $\begin{array}{l}1.77 \\
1.63 \\
2.04 \\
2.45 \\
1.33\end{array}$ & $\begin{array}{l}3.49 \\
3.15 \\
2.88 \\
3.06 \\
3.31\end{array}$ & $\begin{array}{r}11.37 \\
10.91 \\
11.30 \\
10.73 \\
9.75\end{array}$ \\
\hline $\begin{array}{l}1900 \\
1901 \\
1902 \\
1903 \\
1904\end{array}$ & $\begin{array}{r}6.02 \\
6.14 \\
7.31 \\
8.97 \\
10.65\end{array}$ & $\begin{array}{l}3.17 \\
3.74 \\
3.33 \\
3.28 \\
3.96\end{array}$ & $\begin{array}{l}4.47 \\
4.37 \\
5.07 \\
5.78 \\
6.23\end{array}$ & $\begin{array}{l}13.66 \\
14.25 \\
15.71 \\
18.02 \\
20.84\end{array}$ & $\begin{array}{l}5.01 \\
5.24 \\
6.27 \\
7.60 \\
9.40\end{array}$ & $\begin{array}{l}1.48 \\
1.56 \\
1.48 \\
1.02 \\
1.29\end{array}$ & $\begin{array}{l}3.59 \\
3.97 \\
4.54 \\
4.85 \\
4.97\end{array}$ & $\begin{array}{l}10.07 \\
10.76 \\
12.29 \\
13.47 \\
15.66\end{array}$ \\
\hline $\begin{array}{l}1905 \\
1906 \\
1907 \\
1908 \\
1909\end{array}$ & $\begin{array}{l}12.86 \\
14.23 \\
16.30 \\
17.78 \\
21.83\end{array}$ & $\begin{array}{l}4.25 \\
5.38 \\
4.99 \\
5.51 \\
8.41\end{array}$ & $\begin{array}{r}6.72 \\
10.01 \\
11.27 \\
11.87 \\
10.99\end{array}$ & $\begin{array}{l}23.83 \\
29.61 \\
32.56 \\
35.16 \\
41.24\end{array}$ & $\begin{array}{l}10.71 \\
10.26 \\
11.36 \\
12.52 \\
12.89\end{array}$ & $\begin{array}{r}1.33 \\
3.51 \\
7.56 \\
8.86 \\
11.63\end{array}$ & $\begin{array}{l}5.64 \\
6.86 \\
8.00 \\
8.38 \\
9.37\end{array}$ & $\begin{array}{l}17.69 \\
20.63 \\
26.92 \\
29.77 \\
33.88\end{array}$ \\
\hline $\begin{array}{l}1910 \\
1911 \\
1912 \\
1913\end{array}$ & $\begin{array}{l}25.46 \\
26.90 \\
26.34 \\
22.53\end{array}$ & $\begin{array}{l}9.64 \\
6.34 \\
6.67 \\
6.15\end{array}$ & $\begin{array}{l}11.97 \\
13.48 \\
14.72 \\
16.65\end{array}$ & $\begin{array}{l}47.07 \\
46.72 \\
47.73 \\
45.33\end{array}$ & $\begin{array}{l}14.03 \\
15.83 \\
16.68 \\
15.24\end{array}$ & $\begin{array}{r}13.40 \\
12.34 \\
10.16 \\
8.45\end{array}$ & $\begin{array}{l}9.57 \\
9.28 \\
9.55 \\
9.76\end{array}$ & $\begin{array}{l}37.01 \\
37.45 \\
36.39 \\
33.46\end{array}$ \\
\hline
\end{tabular}


Appendix A (cont.)

\begin{tabular}{|c|c|c|c|c|c|c|c|c|}
\hline & $(25)$ & (26) & $\begin{array}{l}(27) \\
\text { es }\end{array}$ & $(28)$ & \multicolumn{4}{|c|}{ Umbria } \\
\hline & $\begin{array}{l}\text { Buil- } \\
\text { dings }\end{array}$ & $\begin{array}{l}\text { Rail- } \\
\text { ways }\end{array}$ & $\begin{array}{l}\text { Other } \\
\text { s.o.c. }\end{array}$ & Total & $\begin{array}{l}\text { Buil- } \\
\text { dings }\end{array}$ & $\begin{array}{l}\text { Rail- } \\
\text { ways }\end{array}$ & $\begin{array}{l}\text { Other } \\
\text { s.o.c. }\end{array}$ & Total \\
\hline $\begin{array}{l}1861 \\
1862 \\
1863 \\
1864\end{array}$ & $\begin{array}{l}.61 \\
.93 \\
.84 \\
.96\end{array}$ & $\begin{array}{l}9.29 \\
8.32 \\
7.99 \\
3.75\end{array}$ & $\begin{array}{l}1.61 \\
1.67 \\
1.34 \\
1.10\end{array}$ & $\begin{array}{r}11.51 \\
10.92 \\
10.17 \\
5.81\end{array}$ & $\begin{array}{r}.84 \\
1.15 \\
1.00 \\
1.06\end{array}$ & $\begin{array}{r}1.60 \\
5.78 \\
9.29 \\
13.21\end{array}$ & $\begin{array}{l}.17 \\
.22 \\
.26 \\
.32\end{array}$ & $\begin{array}{r}2.60 \\
7.15 \\
10.55 \\
14.60\end{array}$ \\
\hline $\begin{array}{l}1865 \\
1866 \\
1867 \\
1868 \\
1869\end{array}$ & $\begin{array}{l}.85 \\
.63 \\
.68 \\
.56 \\
.69\end{array}$ & $\begin{array}{r}4.96 \\
3.88 \\
.00 \\
.00 \\
.25\end{array}$ & $\begin{array}{r}1.80 \\
.92 \\
.97 \\
1.21 \\
1.15\end{array}$ & $\begin{array}{l}7.61 \\
5.42 \\
1.65 \\
1.77 \\
2.09\end{array}$ & $\begin{array}{l}.90 \\
.68 \\
.70 \\
.57 \\
.66\end{array}$ & $\begin{array}{r}16.62 \\
12.03 \\
.00 \\
.00 \\
.00\end{array}$ & $\begin{array}{r}.60 \\
.69 \\
.96 \\
1.24 \\
1.25\end{array}$ & $\begin{array}{r}18.12 \\
13.40 \\
1.66 \\
1.82 \\
1.91\end{array}$ \\
\hline $\begin{array}{l}1870 \\
1871 \\
1872 \\
1873 \\
1874\end{array}$ & $\begin{array}{r}.63 \\
.85 \\
.86 \\
1.43 \\
1.94\end{array}$ & $\begin{array}{l}.50 \\
.73 \\
.97 \\
.80 \\
.03\end{array}$ & $\begin{array}{l}1.22 \\
1.00 \\
1.24 \\
1.42 \\
1.29\end{array}$ & $\begin{array}{l}2.35 \\
2.58 \\
3.06 \\
3.65 \\
3.26\end{array}$ & $\begin{array}{r}.60 \\
.75 \\
.73 \\
1.22 \\
1.66\end{array}$ & $\begin{array}{l}.63 \\
1.79 \\
2.91 \\
4.11 \\
3.98\end{array}$ & $\begin{array}{l}1.42 \\
1.09 \\
1.47 \\
1.55 \\
1.63\end{array}$ & $\begin{array}{l}2.64 \\
3.62 \\
5.11 \\
6.87 \\
7.27\end{array}$ \\
\hline $\begin{array}{l}1875 \\
1876 \\
1877 \\
1878 \\
1879\end{array}$ & $\begin{array}{l}1.37 \\
1.25 \\
1.27 \\
1.13 \\
1.10\end{array}$ & $\begin{array}{l}.05 \\
.07 \\
.06 \\
.06 \\
.04\end{array}$ & $\begin{array}{l}1.13 \\
1.33 \\
1.63 \\
1.70 \\
1.75\end{array}$ & $\begin{array}{l}2.55 \\
2.65 \\
2.95 \\
2.90 \\
2.89\end{array}$ & $\begin{array}{r}1.00 \\
.63 \\
.62 \\
.60 \\
.61\end{array}$ & $\begin{array}{r}1.55 \\
.00 \\
.00 \\
.00 \\
1.40\end{array}$ & $\begin{array}{l}1.69 \\
1.76 \\
2.06 \\
1.97 \\
1.78\end{array}$ & $\begin{array}{l}4.24 \\
2.40 \\
2.68 \\
2.57 \\
3.78\end{array}$ \\
\hline $\begin{array}{l}1880 \\
1881 \\
1882 \\
1883 \\
1884\end{array}$ & $\begin{array}{l}1.14 \\
1.30 \\
1.63 \\
1.74 \\
1.78\end{array}$ & $\begin{array}{r}.63 \\
1.47 \\
3.21 \\
5.08 \\
6.01\end{array}$ & $\begin{array}{l}1.89 \\
1.89 \\
1.85 \\
1.88 \\
2.09\end{array}$ & $\begin{array}{l}3.66 \\
4.66 \\
6.69 \\
8.70 \\
9.88\end{array}$ & $\begin{array}{l}.77 \\
1.07 \\
1.24 \\
1.31 \\
1.82\end{array}$ & $\begin{array}{r}2.69 \\
4.10 \\
5.78 \\
4.67 \\
.13\end{array}$ & $\begin{array}{l}1.74 \\
1.53 \\
1.49 \\
1.58 \\
1.55\end{array}$ & $\begin{array}{l}5.19 \\
6.71 \\
8.51 \\
7.57 \\
3.51\end{array}$ \\
\hline $\begin{array}{l}1885 \\
1886 \\
1887 \\
1888 \\
1889\end{array}$ & $\begin{array}{l}1.82 \\
1.69 \\
1.30 \\
1.03 \\
1.38\end{array}$ & $\begin{array}{r}5.36 \\
4.71 \\
2.62 \\
2.04 \\
.20\end{array}$ & $\begin{array}{l}1.99 \\
2.07 \\
2.57 \\
2.80 \\
2.73\end{array}$ & $\begin{array}{l}9.17 \\
8.47 \\
6.49 \\
5.87 \\
4.31\end{array}$ & $\begin{array}{r}1.79 \\
1.23 \\
.90 \\
.75 \\
.92\end{array}$ & $\begin{array}{l}.45 \\
.32 \\
.00 \\
.03 \\
.05\end{array}$ & $\begin{array}{l}1.38 \\
1.42 \\
1.62 \\
1.72 \\
1.70\end{array}$ & $\begin{array}{l}3.62 \\
2.97 \\
2.52 \\
2.50 \\
2.67\end{array}$ \\
\hline $\begin{array}{l}1890 \\
1891 \\
1892 \\
1893 \\
1894\end{array}$ & $\begin{array}{l}1.87 \\
2.10 \\
1.71 \\
1.65 \\
1.55\end{array}$ & $\begin{array}{r}.16 \\
.64 \\
1.24 \\
1.87 \\
4.74\end{array}$ & $\begin{array}{l}2.46 \\
2.08 \\
1.90 \\
1.68 \\
1.60\end{array}$ & $\begin{array}{l}4.49 \\
4.82 \\
4.85 \\
5.21 \\
7.89\end{array}$ & $\begin{array}{r}1.08 \\
1.22 \\
1.04 \\
.96 \\
.91\end{array}$ & $\begin{array}{l}.06 \\
.02 \\
.01 \\
.00 \\
.02\end{array}$ & $\begin{array}{l}1.93 \\
2.09 \\
1.83 \\
1.60 \\
1.40\end{array}$ & $\begin{array}{l}3.07 \\
3.33 \\
2.89 \\
2.56 \\
2.33\end{array}$ \\
\hline $\begin{array}{l}1895 \\
1896 \\
1897 \\
1898 \\
1899\end{array}$ & $\begin{array}{l}1.70 \\
1.63 \\
1.47 \\
1.38 \\
1.40\end{array}$ & $\begin{array}{r}3.95 \\
2.88 \\
3.85 \\
3.03 \\
.09\end{array}$ & $\begin{array}{l}1.61 \\
1.45 \\
1.55 \\
1.94 \\
2.17\end{array}$ & $\begin{array}{l}7.25 \\
5.96 \\
6.87 \\
6.35 \\
3.66\end{array}$ & $\begin{array}{r}.90 \\
.92 \\
1.25 \\
1.38 \\
1.10\end{array}$ & $\begin{array}{l}.01 \\
.02 \\
.03 \\
.12 \\
.20\end{array}$ & $\begin{array}{l}1.68 \\
2.25 \\
2.90 \\
3.88 \\
5.38\end{array}$ & $\begin{array}{l}2.59 \\
3.19 \\
4.18 \\
5.38 \\
6.67\end{array}$ \\
\hline $\begin{array}{l}1900 \\
1901 \\
1902 \\
1903 \\
1904\end{array}$ & $\begin{array}{l}1.31 \\
1.37 \\
1.87 \\
2.24 \\
2.31\end{array}$ & $\begin{array}{l}.12 \\
.12 \\
.25 \\
.31 \\
.41\end{array}$ & $\begin{array}{l}1.92 \\
1.80 \\
1.94 \\
2.00 \\
1.99\end{array}$ & $\begin{array}{l}3.35 \\
3.29 \\
4.06 \\
4.55 \\
4.71\end{array}$ & $\begin{array}{l}1.34 \\
1.79 \\
1.80 \\
1.26 \\
1.29\end{array}$ & $\begin{array}{l}.18 \\
.35 \\
.10 \\
.07 \\
.08\end{array}$ & $\begin{array}{l}6.15 \\
4.13 \\
2.29 \\
2.08 \\
1.37\end{array}$ & $\begin{array}{l}7.67 \\
6.27 \\
4.18 \\
3.41 \\
2.74\end{array}$ \\
\hline $\begin{array}{l}1905 \\
1906 \\
1907 \\
1908 \\
1909\end{array}$ & $\begin{array}{l}2.49 \\
2.64 \\
2.88 \\
3.03 \\
3.24\end{array}$ & $\begin{array}{r}.53 \\
1.12 \\
.92 \\
.54 \\
.43\end{array}$ & $\begin{array}{l}2.33 \\
2.50 \\
2.52 \\
2.73 \\
3.37\end{array}$ & $\begin{array}{l}5.35 \\
6.26 \\
6.32 \\
6.30 \\
7.04\end{array}$ & $\begin{array}{l}1.39 \\
1.67 \\
2.20 \\
1.99 \\
2.06\end{array}$ & $\begin{array}{l}.10 \\
.13 \\
.16 \\
.23 \\
.33\end{array}$ & $\begin{array}{l}2.49 \\
4.01 \\
4.88 \\
4.39 \\
4.03\end{array}$ & $\begin{array}{l}3.98 \\
5.81 \\
7.25 \\
6.61 \\
6.41\end{array}$ \\
\hline $\begin{array}{l}1910 \\
1911 \\
1912 \\
1913\end{array}$ & $\begin{array}{l}3.40 \\
2.93 \\
2.88 \\
3.22\end{array}$ & $\begin{array}{r}.29 \\
.43 \\
1.80 \\
3.38\end{array}$ & $\begin{array}{l}4.00 \\
4.12 \\
4.77 \\
4.59\end{array}$ & $\begin{array}{r}7.69 \\
7.48 \\
9.45 \\
11.19\end{array}$ & $\begin{array}{l}2.54 \\
2.86 \\
4.07 \\
7.17\end{array}$ & $\begin{array}{l}.17 \\
.21 \\
.23 \\
.51\end{array}$ & $\begin{array}{l}4.91 \\
7.22 \\
7.21 \\
4.57\end{array}$ & $\begin{array}{r}7.62 \\
10.29 \\
11.51 \\
12.25\end{array}$ \\
\hline
\end{tabular}




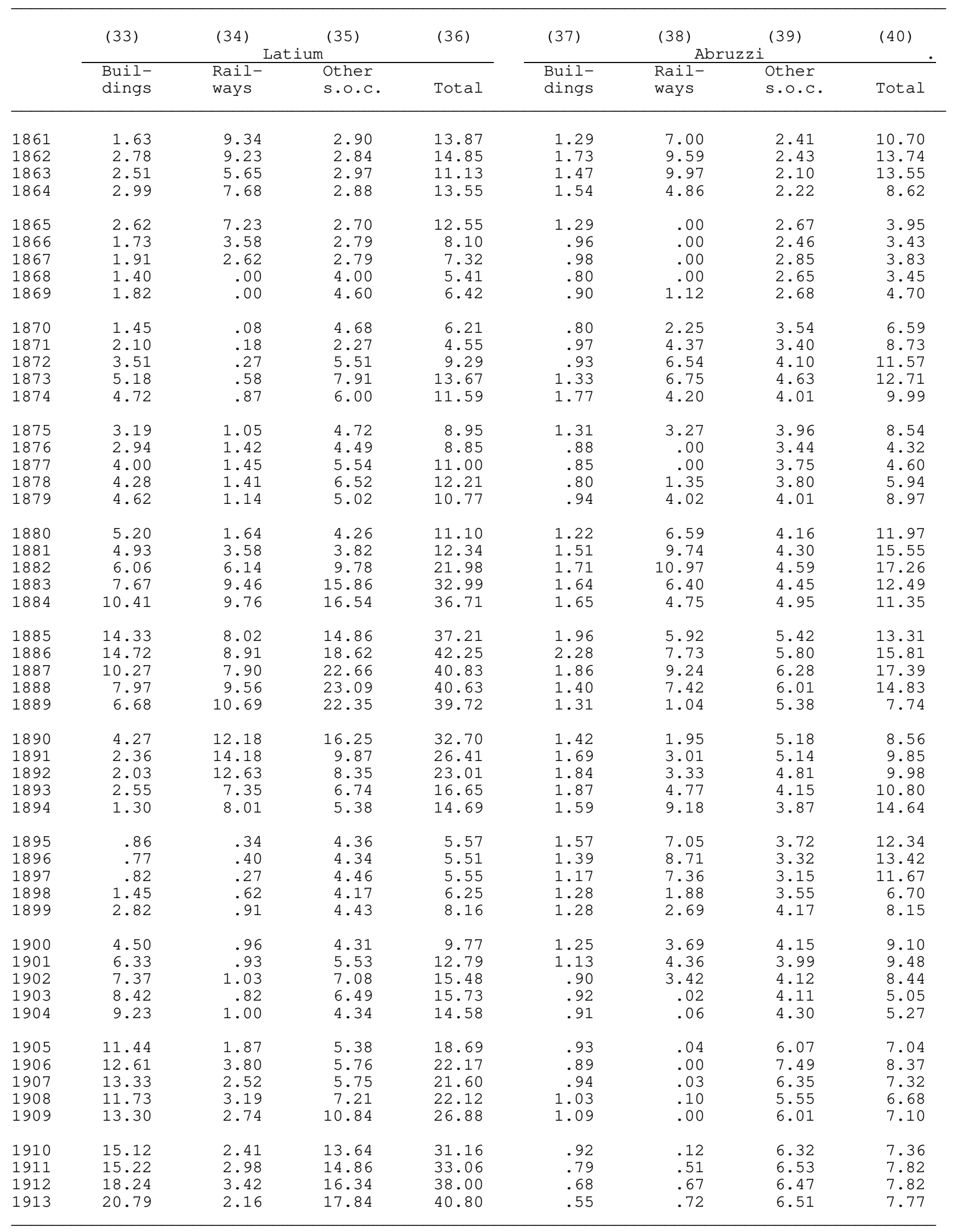


Appendix A (cont.)

\begin{tabular}{|c|c|c|c|c|c|c|c|c|}
\hline & $(41)$ & ${ }^{(42)} \mathrm{Ca}$ & $\begin{array}{l}(43) \\
i a\end{array}$ & $(44)$ & $(45)$ & $(46)_{A}$ & $a^{(47)}$ & (48) \\
\hline & Buil- & Rail- & Other & & Buil- & Rail- & Other & \\
\hline & dings & ways & S.O.C. & Total & dings & ways & S.O.C. & Total \\
\hline 1861 & 2.77 & 6.97 & 3.36 & 13.10 & 2.99 & 9.26 & .95 & 13.19 \\
\hline 1862 & 4.73 & 5.14 & 3.63 & 13.51 & 5.26 & 16.22 & .98 & 22.46 \\
\hline 1863 & 4.28 & 4.75 & 3.67 & 12.70 & 4.76 & 23.33 & .96 & 29.04 \\
\hline 1864 & 5.11 & 3.55 & 3.00 & 11.65 & 5.74 & 28.04 & 1.00 & 34.78 \\
\hline 1865 & 4.49 & 6.61 & 3.65 & 14.75 & 5.05 & 21.49 & 1.46 & 28.00 \\
\hline 1866 & 3.02 & 9.46 & 4.94 & 17.42 & 3.31 & 13.33 & 1.70 & 18.34 \\
\hline 1867 & 3.34 & 10.21 & 4.37 & 17.91 & 3.68 & 12.92 & 2.13 & 18.72 \\
\hline 1868 & 2.51 & 6.84 & 3.86 & 13.20 & 2.69 & 9.72 & 3.04 & 15.45 \\
\hline 1869 & 3.24 & 1.35 & 4.31 & 8.90 & 3.53 & 2.73 & 3.31 & 9.57 \\
\hline 1870 & 2.65 & .58 & 6.07 & 9.30 & 2.79 & .97 & 4.07 & 7.82 \\
\hline 1871 & 3.81 & 1.02 & 6.21 & 11.04 & 4.10 & 1.19 & 2.87 & 8.16 \\
\hline 1872 & 3.60 & 1.70 & 6.51 & 11.81 & 3.79 & .93 & 3.91 & 8.63 \\
\hline 1873 & 5.08 & 2.63 & 5.99 & 13.70 & 5.15 & .05 & 4.47 & 9.68 \\
\hline 1874 & 6.21 & 2.86 & 5.98 & 15.05 & 5.56 & .10 & 3.77 & 9.43 \\
\hline 1875 & 4.66 & 2.25 & 6.40 & 13.31 & 3.91 & .16 & 2.95 & 7.02 \\
\hline 1876 & 3.34 & 1.72 & 8.87 & 13.93 & 3.74 & .21 & 3.10 & 7.04 \\
\hline 1877 & 3.59 & 2.12 & 12.25 & 17.95 & 3.44 & .18 & 3.70 & 7.32 \\
\hline 1878 & 3.52 & 3.52 & 10.78 & 17.82 & 3.16 & .16 & 3.96 & 7.28 \\
\hline 1879 & 2.94 & 4.34 & 8.77 & 16.06 & 3.11 & .12 & 3.98 & 7.21 \\
\hline 1880 & 2.94 & 4.67 & 8.80 & 16.41 & 3.10 & .71 & 3.64 & 7.45 \\
\hline 1881 & 3.67 & 7.60 & 7.24 & 18.52 & 3.71 & 3.06 & 2.69 & 9.46 \\
\hline 1882 & 4.67 & 9.32 & 7.15 & 21.15 & 4.75 & 7.27 & 2.83 & 14.85 \\
\hline 1883 & 4.92 & 9.16 & 9.15 & 23.23 & 5.71 & 9.42 & 3.24 & 18.37 \\
\hline 1884 & 4.80 & 9.67 & 12.53 & 27.00 & 6.70 & 11.43 & 4.14 & 22.27 \\
\hline 1885 & 4.65 & 11.32 & 11.30 & 27.27 & 6.96 & 10.30 & 4.30 & 21.56 \\
\hline 1886 & 5.64 & 9.24 & 9.37 & 24.25 & 5.85 & 6.21 & 4.08 & 16.14 \\
\hline 1887 & 6.52 & 6.69 & 9.46 & 22.67 & 3.31 & 1.99 & 4.61 & 9.91 \\
\hline 1888 & 6.20 & 7.70 & 9.77 & 23.67 & 2.29 & 3.15 & 5.80 & 11.24 \\
\hline 1889 & 5.67 & 8.10 & 9.74 & 23.51 & 2.16 & 4.92 & 6.13 & 13.21 \\
\hline 1890 & 6.21 & 9.33 & 9.19 & 24.73 & 2.77 & 6.19 & 5.56 & 14.51 \\
\hline 1891 & 8.81 & 12.18 & 8.27 & 29.25 & 3.81 & 8.26 & 4.48 & 16.55 \\
\hline 1892 & 8.90 & 12.47 & 7.57 & 28.94 & 3.87 & 7.50 & 3.52 & 14.89 \\
\hline 1893 & 10.36 & 9.85 & 7.20 & 27.41 & 4.97 & 3.93 & 3.19 & 12.09 \\
\hline 1894 & 7.82 & 12.60 & 6.57 & 26.99 & 5.69 & 7.11 & 2.91 & 15.71 \\
\hline 1895 & 5.50 & 5.18 & 6.35 & 17.03 & 5.73 & 4.02 & 2.36 & 12.11 \\
\hline 1896 & 4.43 & .15 & 5.78 & 10.36 & 4.74 & .07 & 1.93 & 6.74 \\
\hline 1897 & 3.38 & .19 & 5.43 & 8.99 & 3.75 & .18 & 1.87 & 5.80 \\
\hline 1898 & 2.97 & 1.00 & 5.32 & 9.30 & 4.03 & .57 & 2.03 & 6.63 \\
\hline 1899 & 2.78 & 1.98 & 5.37 & 10.12 & 4.56 & .51 & 2.25 & 7.33 \\
\hline 1900 & 3.41 & 2.32 & 6.05 & 11.77 & 4.48 & .55 & 2.29 & 7.32 \\
\hline 1901 & 3.17 & 3.84 & 6.82 & 13.83 & 3.91 & .43 & 2.35 & 6.69 \\
\hline 1902 & 2.35 & 3.54 & 8.06 & 13.95 & 2.95 & .68 & 2.76 & 6.39 \\
\hline 1903 & 2.43 & 1.39 & 9.39 & 13.20 & 2.41 & .61 & 2.96 & 5.99 \\
\hline 1904 & 2.62 & 1.60 & 8.90 & 13.12 & 3.02 & .59 & 2.84 & 6.45 \\
\hline 1905 & 3.22 & .82 & 8.86 & 12.90 & 4.51 & .72 & 2.97 & 8.20 \\
\hline 1906 & 3.86 & 1.37 & 11.29 & 16.52 & 5.46 & 1.05 & 3.67 & 10.18 \\
\hline 1907 & 4.58 & 1.41 & 12.88 & 18.87 & 5.92 & 1.00 & 4.15 & 11.07 \\
\hline 1908 & 5.96 & 2.09 & 14.01 & 22.06 & 6.43 & .92 & 4.88 & 12.22 \\
\hline 1909 & 7.79 & 2.13 & 15.81 & 25.73 & 7.43 & 1.45 & 5.86 & 14.74 \\
\hline 1910 & 8.59 & 1.92 & 16.87 & 27.38 & 8.97 & 1.69 & 7.28 & 17.94 \\
\hline 1911 & 9.14 & 2.74 & 17.54 & 29.42 & 10.64 & 1.32 & 8.03 & 19.99 \\
\hline 1912 & 11.76 & 3.27 & 17.43 & 32.46 & 11.06 & 3.03 & 13.97 & 28.06 \\
\hline 1913 & 11.71 & 3.24 & 15.32 & 30.27 & 9.69 & 5.40 & 15.86 & 30.95 \\
\hline
\end{tabular}


Appendix A (cont.)

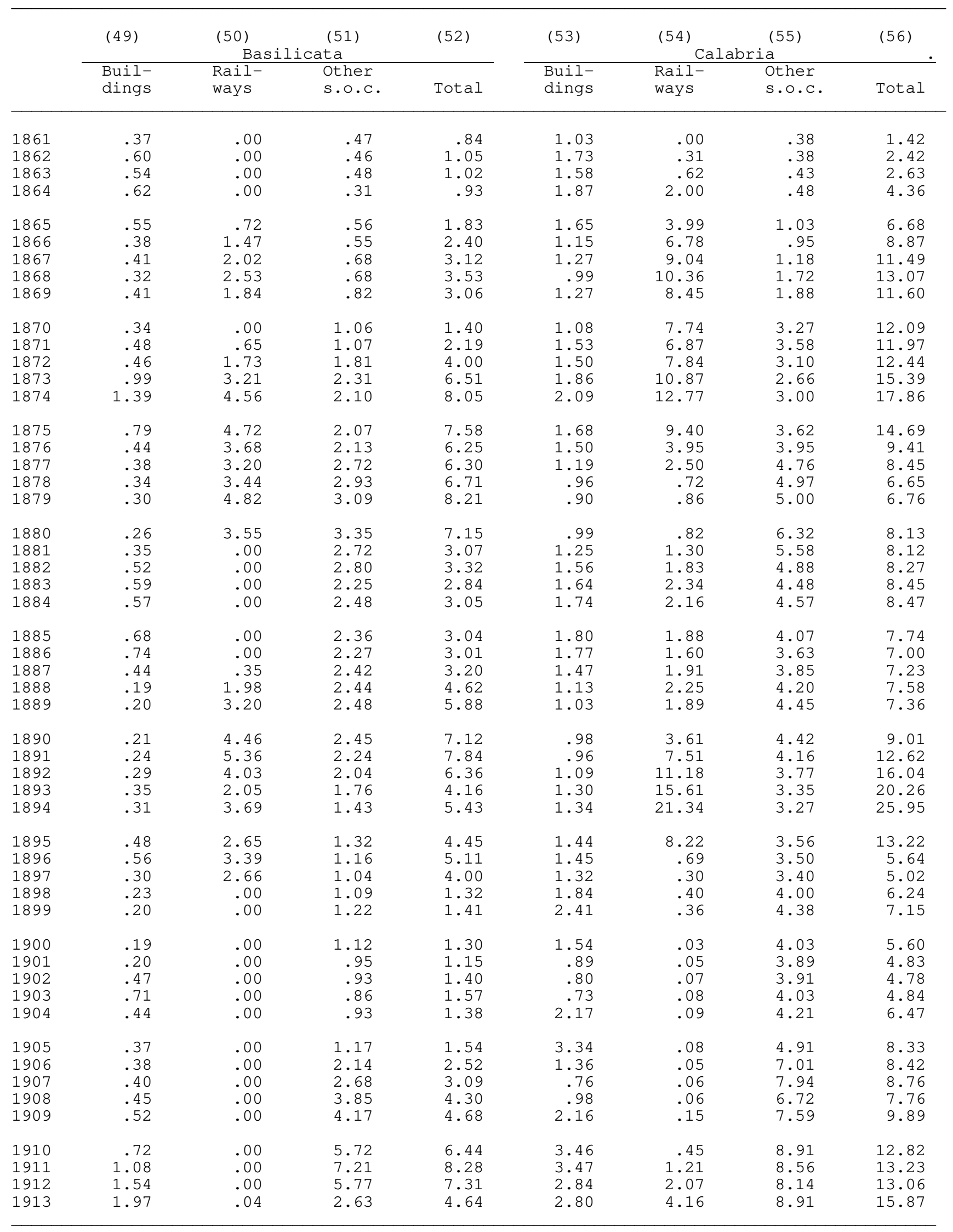




\begin{tabular}{|c|c|c|c|c|c|c|c|c|}
\hline & $(57)$ & (58) & (59) & $(60)$ & $(61)$ & $(62)$ & (63) & $(64)$ \\
\hline & & & & & & & & \\
\hline & Buil- & Rail- & Other & & Buil- & Rail- & Other & \\
\hline & dings & ways & S.O.C. & Total & dings & ways & S.O.C. & Total \\
\hline 1861 & 3.42 & 1.40 & 2.44 & 7.27 & .74 & .00 & 1.27 & 2.01 \\
\hline 1862 & 6.34 & 2.89 & 2.17 & 11.39 & 1.34 & .00 & 1.35 & 2.69 \\
\hline 1863 & 5.85 & 4.85 & 2.59 & 13.29 & 1.22 & .00 & 2.04 & 3.26 \\
\hline 1864 & 7.25 & 5.59 & 2.90 & 15.75 & 1.49 & .00 & 2.17 & 3.66 \\
\hline 1865 & 6.47 & 7.36 & 3.88 & 17.71 & 1.32 & .00 & 1.76 & 3.08 \\
\hline 1866 & 4.25 & 10.15 & 4.38 & 18.78 & .87 & .00 & 1.85 & 2.72 \\
\hline 1867 & 4.82 & 9.68 & 6.05 & 20.54 & .97 & .93 & 1.93 & 3.84 \\
\hline 1868 & 3.57 & 10.07 & 6.75 & 20.39 & .72 & 3.59 & 1.91 & 6.22 \\
\hline 1869 & 4.78 & 10.89 & 6.39 & 22.06 & .95 & 5.85 & 1.77 & 8.57 \\
\hline 1870 & 3.87 & 8.68 & 7.47 & 20.01 & .76 & 8.94 & 2.49 & 12.19 \\
\hline 1871 & 5.76 & 4.71 & 6.27 & 16.74 & 1.12 & 10.75 & 2.21 & 14.08 \\
\hline 1872 & 5.48 & 4.24 & 6.37 & 16.09 & 1.05 & 7.53 & 2.79 & 11.37 \\
\hline 1873 & 8.17 & 7.01 & 5.81 & 20.99 & 1.47 & 3.24 & 3.18 & 7.89 \\
\hline 1874 & 8.09 & 8.04 & 6.02 & 22.15 & 1.83 & 3.08 & 2.55 & 7.46 \\
\hline 1875 & 5.76 & 7.08 & 6.25 & 19.09 & 1.29 & 1.20 & 2.18 & 4.66 \\
\hline 1876 & 4.77 & 7.89 & 7.28 & 19.94 & 1.10 & 3.93 & 2.40 & 7.43 \\
\hline 1877 & 4.20 & 8.63 & 9.11 & 21.95 & .99 & 6.56 & 3.02 & 10.57 \\
\hline 1878 & 3.45 & 13.79 & 8.85 & 26.08 & .66 & 8.75 & 2.68 & 12.10 \\
\hline 1879 & 3.32 & 19.11 & 8.86 & 31.29 & .69 & 10.74 & 2.23 & 13.66 \\
\hline 1880 & 3.70 & 17.78 & 9.65 & 31.14 & .90 & 9.04 & 2.16 & 12.10 \\
\hline 1881 & 4.39 & 7.73 & 8.43 & 20.55 & 1.12 & 2.49 & 2.08 & 5.70 \\
\hline 1882 & 5.35 & 1.64 & 8.59 & 15.59 & 1.13 & 1.79 & 1.68 & 4.61 \\
\hline 1883 & 6.07 & 3.08 & 8.73 & 17.88 & 1.12 & 1.45 & 1.56 & 4.13 \\
\hline 1884 & 6.54 & 4.26 & 9.37 & 20.16 & 1.29 & .00 & 1.58 & 2.87 \\
\hline 1885 & 7.52 & 6.41 & 9.06 & 22.99 & 1.30 & .00 & 1.59 & 2.89 \\
\hline 1886 & 8.52 & 7.11 & 8.79 & 24.42 & 1.21 & .30 & 1.95 & 3.46 \\
\hline 1887 & 6.95 & 8.66 & 9.44 & 25.04 & 1.32 & 1.37 & 2.68 & 5.37 \\
\hline 1888 & 4.97 & 12.16 & 9.47 & 26.60 & 1.23 & 1.68 & 3.45 & 6.36 \\
\hline 1889 & 5.50 & 15.87 & 8.65 & 30.02 & .75 & .67 & 4.10 & 5.52 \\
\hline 1890 & 5.74 & 17.40 & 9.43 & 32.58 & 1.26 & .15 & 3.66 & 5.07 \\
\hline 1891 & 4.75 & 18.46 & 9.77 & 32.99 & 1.83 & .39 & 2.26 & 4.48 \\
\hline 1892 & 5.62 & 15.11 & 7.87 & 28.60 & 1.71 & 1.24 & 1.46 & 4.40 \\
\hline 1893 & 7.41 & 11.59 & 7.48 & 26.48 & 1.99 & 1.41 & 1.19 & 4.59 \\
\hline 1894 & 8.22 & 8.04 & 7.92 & 24.18 & 1.30 & .30 & 1.14 & 2.74 \\
\hline 1895 & 7.38 & 3.51 & 6.68 & 17.58 & .99 & .00 & 1.03 & 2.01 \\
\hline 1896 & 6.88 & .13 & 6.99 & 14.01 & 1.16 & .02 & 1.18 & 2.36 \\
\hline 1897 & 6.15 & .14 & 8.56 & 14.85 & .96 & .04 & 1.42 & 2.42 \\
\hline 1898 & 4.82 & .47 & 8.17 & 13.46 & 1.08 & .04 & 1.25 & 2.36 \\
\hline 1899 & 3.83 & .96 & 7.91 & 12.70 & 1.50 & .03 & 1.28 & 2.81 \\
\hline 1900 & 3.22 & .95 & 8.38 & 12.55 & 1.49 & .04 & 1.43 & 2.96 \\
\hline 1901 & 2.83 & .60 & 8.39 & 11.82 & 1.39 & .04 & 1.34 & 2.77 \\
\hline 1902 & 2.80 & .78 & 8.56 & 12.14 & .88 & .05 & 1.40 & 2.33 \\
\hline 1903 & 3.23 & .56 & 8.83 & 12.62 & .81 & .03 & 1.66 & 2.51 \\
\hline 1904 & 3.92 & .53 & 8.65 & 13.10 & .90 & .04 & 1.79 & 2.73 \\
\hline 1905 & 4.11 & .93 & 8.99 & 14.02 & 1.22 & .04 & 1.83 & 3.09 \\
\hline 1906 & 4.00 & .60 & 9.76 & 14.36 & 1.50 & .06 & 1.98 & 3.54 \\
\hline 1907 & 4.64 & .74 & 10.70 & 16.08 & 1.48 & .07 & 2.08 & 3.63 \\
\hline 1908 & 5.98 & .79 & 12.52 & 19.28 & 2.16 & .07 & 2.21 & 4.44 \\
\hline 1909 & 13.54 & .85 & 19.01 & 33.40 & 6.57 & .06 & 2.83 & 9.46 \\
\hline 1910 & 14.90 & 1.67 & 22.29 & 38.86 & 7.62 & .08 & 3.05 & 10.74 \\
\hline 1911 & 15.43 & 3.06 & 19.54 & 38.03 & 7.67 & .09 & 3.10 & 10.86 \\
\hline 1912 & 16.00 & 2.02 & 17.32 & 35.34 & 7.05 & .18 & 3.49 & 10.72 \\
\hline 1913 & 16.63 & 1.89 & 16.48 & 35.00 & 6.74 & .61 & 3.54 & 10.88 \\
\hline
\end{tabular}

Source: see text. 
Appendix B: Maintenance, 1861-1913: value added at 1911 prices (million lire)

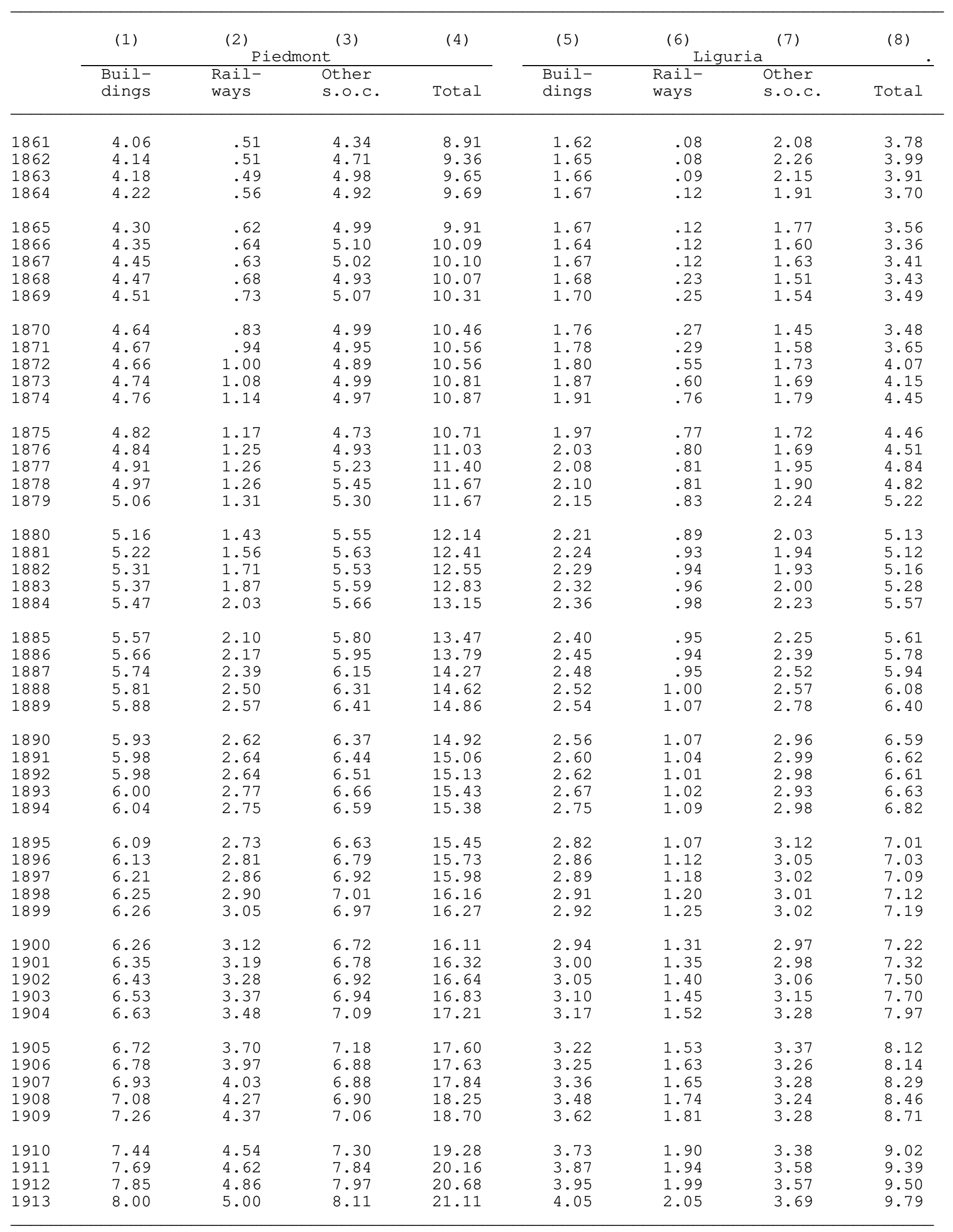




\begin{tabular}{|c|c|c|c|c|c|c|c|c|}
\hline & \multicolumn{4}{|c|}{ Lombardy } & \multicolumn{4}{|c|}{ Venetia } \\
\hline & $\begin{array}{l}\text { Buil- } \\
\text { dings }\end{array}$ & $\begin{array}{l}\text { Rail- } \\
\text { ways }\end{array}$ & $\begin{array}{l}\text { Other } \\
\text { s.o.c. }\end{array}$ & Total & $\begin{array}{l}\text { Buil- } \\
\text { dings }\end{array}$ & $\begin{array}{l}\text { Rail- } \\
\text { ways }\end{array}$ & $\begin{array}{l}\text { Other } \\
\text { s.o.c. }\end{array}$ & Total \\
\hline $\begin{array}{l}1861 \\
1862 \\
1863 \\
1864\end{array}$ & $\begin{array}{l}5.30 \\
5.40 \\
5.45 \\
5.49\end{array}$ & $\begin{array}{l}.48 \\
.56 \\
.66 \\
.70\end{array}$ & $\begin{array}{l}6.06 \\
6.58 \\
6.64 \\
6.56\end{array}$ & $\begin{array}{l}11.84 \\
12.53 \\
12.74 \\
12.76\end{array}$ & $\begin{array}{l}5.05 \\
5.15 \\
5.19 \\
5.24\end{array}$ & $\begin{array}{l}.27 \\
.27 \\
.26 \\
.27\end{array}$ & $\begin{array}{l}9.01 \\
9.31 \\
9.77 \\
9.41\end{array}$ & $\begin{array}{l}14.34 \\
14.73 \\
15.22 \\
14.93\end{array}$ \\
\hline $\begin{array}{l}1865 \\
1866 \\
1867 \\
1868 \\
1869\end{array}$ & $\begin{array}{l}5.49 \\
5.44 \\
5.53 \\
5.55 \\
5.59\end{array}$ & $\begin{array}{r}.76 \\
.90 \\
.92 \\
.96 \\
1.03\end{array}$ & $\begin{array}{l}6.65 \\
6.03 \\
5.70 \\
6.07 \\
6.34\end{array}$ & $\begin{array}{l}12.90 \\
12.36 \\
12.15 \\
12.58 \\
12.96\end{array}$ & $\begin{array}{l}5.22 \\
5.13 \\
5.16 \\
5.14 \\
5.13\end{array}$ & $\begin{array}{l}.28 \\
.34 \\
.34 \\
.35 \\
.38\end{array}$ & $\begin{array}{r}9.34 \\
7.58 \\
10.98 \\
8.46 \\
7.37\end{array}$ & $\begin{array}{l}14.84 \\
13.04 \\
16.48 \\
13.95 \\
12.88\end{array}$ \\
\hline $\begin{array}{l}1870 \\
1871 \\
1872 \\
1873 \\
1874\end{array}$ & $\begin{array}{l}5.77 \\
5.79 \\
5.79 \\
5.90 \\
5.93\end{array}$ & $\begin{array}{l}1.16 \\
1.25 \\
1.32 \\
1.50 \\
1.55\end{array}$ & $\begin{array}{l}6.14 \\
6.37 \\
6.72 \\
6.49 \\
7.06\end{array}$ & $\begin{array}{l}13.07 \\
13.41 \\
13.82 \\
13.89 \\
14.54\end{array}$ & $\begin{array}{l}5.23 \\
5.21 \\
5.17 \\
5.22 \\
5.21\end{array}$ & $\begin{array}{l}.39 \\
.42 \\
.45 \\
.48 \\
.46\end{array}$ & $\begin{array}{l}6.89 \\
7.16 \\
7.93 \\
7.59 \\
8.37\end{array}$ & $\begin{array}{l}12.51 \\
12.80 \\
13.55 \\
13.29 \\
14.04\end{array}$ \\
\hline $\begin{array}{l}1875 \\
1876 \\
1877 \\
1878 \\
1879\end{array}$ & $\begin{array}{l}5.99 \\
6.03 \\
6.13 \\
6.21 \\
6.32\end{array}$ & $\begin{array}{l}1.57 \\
1.71 \\
1.72 \\
1.78 \\
1.98\end{array}$ & $\begin{array}{l}6.69 \\
6.69 \\
7.13 \\
7.29 \\
7.36\end{array}$ & $\begin{array}{l}14.25 \\
14.43 \\
14.98 \\
15.28 \\
15.66\end{array}$ & $\begin{array}{l}5.24 \\
5.23 \\
5.29 \\
5.32 \\
5.37\end{array}$ & $\begin{array}{l}.50 \\
.63 \\
.83 \\
.84 \\
.89\end{array}$ & $\begin{array}{l}7.70 \\
8.15 \\
8.44 \\
8.77 \\
8.86\end{array}$ & $\begin{array}{l}13.44 \\
14.01 \\
14.56 \\
14.93 \\
15.13\end{array}$ \\
\hline $\begin{array}{l}1880 \\
1881 \\
1882 \\
1883 \\
1884\end{array}$ & $\begin{array}{l}6.41 \\
6.46 \\
6.55 \\
6.59 \\
6.70\end{array}$ & $\begin{array}{l}2.17 \\
2.32 \\
2.64 \\
2.73 \\
2.98\end{array}$ & $\begin{array}{l}7.86 \\
7.83 \\
7.71 \\
8.34 \\
7.28\end{array}$ & $\begin{array}{l}16.44 \\
16.61 \\
16.90 \\
17.66 \\
16.96\end{array}$ & $\begin{array}{l}5.41 \\
5.40 \\
5.43 \\
5.43 \\
5.48\end{array}$ & $\begin{array}{l}.97 \\
1.02 \\
1.04 \\
1.08 \\
1.15\end{array}$ & $\begin{array}{r}9.25 \\
8.70 \\
12.03 \\
10.76 \\
9.30\end{array}$ & $\begin{array}{l}15.63 \\
15.12 \\
18.50 \\
17.27 \\
15.93\end{array}$ \\
\hline $\begin{array}{l}1885 \\
1886 \\
1887 \\
1888 \\
1889\end{array}$ & $\begin{array}{l}6.83 \\
6.96 \\
7.07 \\
7.16 \\
7.21\end{array}$ & $\begin{array}{l}3.18 \\
3.30 \\
3.55 \\
3.89 \\
3.98\end{array}$ & $\begin{array}{l}7.10 \\
7.76 \\
7.85 \\
7.78 \\
8.33\end{array}$ & $\begin{array}{l}17.11 \\
18.02 \\
18.47 \\
18.83 \\
19.52\end{array}$ & $\begin{array}{l}5.55 \\
5.61 \\
5.66 \\
5.71 \\
5.72\end{array}$ & $\begin{array}{l}1.29 \\
1.51 \\
1.58 \\
1.72 \\
1.78\end{array}$ & $\begin{array}{r}9.01 \\
9.82 \\
9.80 \\
9.84 \\
10.28\end{array}$ & $\begin{array}{l}15.85 \\
16.94 \\
17.03 \\
17.27 \\
17.78\end{array}$ \\
\hline $\begin{array}{l}1890 \\
1891 \\
1892 \\
1893 \\
1894\end{array}$ & $\begin{array}{l}7.24 \\
7.28 \\
7.26 \\
7.32 \\
7.45\end{array}$ & $\begin{array}{l}4.04 \\
4.00 \\
3.99 \\
4.15 \\
4.21\end{array}$ & $\begin{array}{l}8.30 \\
8.12 \\
8.33 \\
8.49 \\
8.10\end{array}$ & $\begin{array}{l}19.58 \\
19.39 \\
19.58 \\
19.96 \\
19.76\end{array}$ & $\begin{array}{l}5.71 \\
5.72 \\
5.69 \\
5.69 \\
5.72\end{array}$ & $\begin{array}{l}1.81 \\
1.77 \\
1.74 \\
1.78 \\
1.76\end{array}$ & $\begin{array}{r}9.93 \\
9.77 \\
9.99 \\
10.13 \\
9.68\end{array}$ & $\begin{array}{l}17.45 \\
17.25 \\
17.43 \\
17.60 \\
17.15\end{array}$ \\
\hline $\begin{array}{l}1895 \\
1896 \\
1897 \\
1898 \\
1899\end{array}$ & $\begin{array}{l}7.57 \\
7.67 \\
7.82 \\
7.94 \\
8.01\end{array}$ & $\begin{array}{l}4.17 \\
4.31 \\
4.46 \\
4.58 \\
4.73\end{array}$ & $\begin{array}{l}8.27 \\
8.84 \\
8.98 \\
9.42 \\
9.44\end{array}$ & $\begin{array}{l}20.01 \\
20.82 \\
21.26 \\
21.94 \\
22.18\end{array}$ & $\begin{array}{l}5.76 \\
5.78 \\
5.85 \\
5.91 \\
5.90\end{array}$ & $\begin{array}{l}1.73 \\
1.79 \\
1.84 \\
1.86 \\
1.95\end{array}$ & $\begin{array}{r}9.79 \\
10.18 \\
10.25 \\
10.53 \\
10.22\end{array}$ & $\begin{array}{l}17.27 \\
17.75 \\
17.95 \\
18.30 \\
18.07\end{array}$ \\
\hline $\begin{array}{l}1900 \\
1901 \\
1902 \\
1903 \\
1904\end{array}$ & $\begin{array}{l}8.11 \\
8.31 \\
8.49 \\
8.64 \\
8.83\end{array}$ & $\begin{array}{l}4.80 \\
4.90 \\
5.13 \\
5.28 \\
5.55\end{array}$ & $\begin{array}{r}9.04 \\
9.26 \\
9.67 \\
9.81 \\
10.05\end{array}$ & $\begin{array}{l}21.95 \\
22.48 \\
23.29 \\
23.72 \\
24.43\end{array}$ & $\begin{array}{l}5.91 \\
5.96 \\
6.01 \\
6.07 \\
6.17\end{array}$ & $\begin{array}{l}1.99 \\
2.02 \\
2.08 \\
2.15 \\
2.25\end{array}$ & $\begin{array}{l}9.51 \\
9.51 \\
9.91 \\
9.90 \\
9.97\end{array}$ & $\begin{array}{l}17.40 \\
17.49 \\
18.00 \\
18.11 \\
18.38\end{array}$ \\
\hline $\begin{array}{l}1905 \\
1906 \\
1907 \\
1908 \\
1909\end{array}$ & $\begin{array}{r}9.05 \\
9.26 \\
9.59 \\
9.92 \\
10.27\end{array}$ & $\begin{array}{l}5.65 \\
6.01 \\
6.15 \\
6.50 \\
6.79\end{array}$ & $\begin{array}{l}10.33 \\
10.07 \\
10.15 \\
10.14 \\
10.33\end{array}$ & $\begin{array}{l}25.03 \\
25.34 \\
25.89 \\
26.56 \\
27.39\end{array}$ & $\begin{array}{l}6.28 \\
6.36 \\
6.51 \\
6.67 \\
6.85\end{array}$ & $\begin{array}{l}2.27 \\
2.39 \\
2.43 \\
2.67 \\
2.79\end{array}$ & $\begin{array}{r}10.09 \\
9.60 \\
9.56 \\
9.53 \\
9.78\end{array}$ & $\begin{array}{l}18.63 \\
18.35 \\
18.50 \\
18.87 \\
19.42\end{array}$ \\
\hline $\begin{array}{l}1910 \\
1911 \\
1912 \\
1913\end{array}$ & $\begin{array}{l}10.61 \\
11.10 \\
11.45 \\
11.84\end{array}$ & $\begin{array}{l}7.08 \\
7.44 \\
7.64 \\
7.82\end{array}$ & $\begin{array}{l}10.64 \\
11.29 \\
11.40 \\
11.46\end{array}$ & $\begin{array}{l}28.33 \\
29.83 \\
30.50 \\
31.12\end{array}$ & $\begin{array}{l}7.03 \\
7.28 \\
7.45 \\
7.64\end{array}$ & $\begin{array}{l}3.03 \\
3.16 \\
3.36 \\
3.57\end{array}$ & $\begin{array}{l}10.18 \\
10.83 \\
10.95 \\
10.93\end{array}$ & $\begin{array}{l}20.24 \\
21.27 \\
21.77 \\
22.15\end{array}$ \\
\hline
\end{tabular}




\begin{tabular}{|c|c|c|c|c|c|c|c|c|}
\hline & $(17)$ & (18) & Emilia & $(20)$ & (21) & \multicolumn{3}{|c|}{ Tuscany } \\
\hline & $\begin{array}{l}\text { Buil- } \\
\text { dings }\end{array}$ & $\begin{array}{l}\text { Rail- } \\
\text { ways }\end{array}$ & $\begin{array}{l}\text { Other } \\
\text { s.o.c. }\end{array}$ & Total & $\begin{array}{l}\text { Buil- } \\
\text { dings }\end{array}$ & $\begin{array}{l}\text { Rail- } \\
\text { ways }\end{array}$ & $\begin{array}{l}\text { Other } \\
\text { s.o.c. }\end{array}$ & Total \\
\hline $\begin{array}{l}1861 \\
1862 \\
1863 \\
1864\end{array}$ & $\begin{array}{l}4.71 \\
4.78 \\
4.80 \\
4.83\end{array}$ & $\begin{array}{l}.35 \\
.44 \\
.52 \\
.54\end{array}$ & $\begin{array}{l}5.93 \\
6.37 \\
6.25 \\
6.25\end{array}$ & $\begin{array}{l}10.99 \\
11.59 \\
11.57 \\
11.62\end{array}$ & $\begin{array}{l}4.88 \\
4.97 \\
5.00 \\
5.03\end{array}$ & $\begin{array}{l}.35 \\
.39 \\
.55 \\
.69\end{array}$ & $\begin{array}{l}4.53 \\
4.97 \\
5.22 \\
4.88\end{array}$ & $\begin{array}{r}9.77 \\
10.32 \\
10.77 \\
10.60\end{array}$ \\
\hline $\begin{array}{l}1865 \\
1866 \\
1867 \\
1868 \\
1869\end{array}$ & $\begin{array}{l}4.80 \\
4.72 \\
4.74 \\
4.70 \\
4.68\end{array}$ & $\begin{array}{l}.56 \\
.56 \\
.55 \\
.58 \\
.62\end{array}$ & $\begin{array}{l}6.22 \\
6.66 \\
6.46 \\
6.55 \\
6.67\end{array}$ & $\begin{array}{l}11.59 \\
11.94 \\
11.75 \\
11.83 \\
11.97\end{array}$ & $\begin{array}{l}4.99 \\
4.89 \\
4.91 \\
4.86 \\
4.84\end{array}$ & $\begin{array}{l}.73 \\
.80 \\
.80 \\
.84 \\
.90\end{array}$ & $\begin{array}{l}4.71 \\
5.04 \\
4.77 \\
5.05 \\
5.30\end{array}$ & $\begin{array}{l}10.43 \\
10.73 \\
10.48 \\
10.76 \\
11.04\end{array}$ \\
\hline $\begin{array}{l}1870 \\
1871 \\
1872 \\
1873 \\
1874\end{array}$ & $\begin{array}{l}4.75 \\
4.72 \\
4.68 \\
4.72 \\
4.71\end{array}$ & $\begin{array}{l}.65 \\
.70 \\
.79 \\
.85 \\
.82\end{array}$ & $\begin{array}{l}6.38 \\
6.54 \\
6.59 \\
6.66 \\
7.29\end{array}$ & $\begin{array}{l}11.78 \\
11.96 \\
12.07 \\
12.23 \\
12.81\end{array}$ & $\begin{array}{l}4.93 \\
4.90 \\
4.86 \\
4.91 \\
4.90\end{array}$ & $\begin{array}{r}.94 \\
1.03 \\
1.15 \\
1.25 \\
1.21\end{array}$ & $\begin{array}{l}5.42 \\
5.42 \\
5.33 \\
5.16 \\
5.38\end{array}$ & $\begin{array}{l}11.30 \\
11.35 \\
11.34 \\
11.31 \\
11.49\end{array}$ \\
\hline $\begin{array}{l}1875 \\
1876 \\
1877 \\
1878 \\
1879\end{array}$ & $\begin{array}{l}4.72 \\
4.71 \\
4.75 \\
4.78 \\
4.82\end{array}$ & $\begin{array}{l}.83 \\
.86 \\
.86 \\
.86 \\
.88\end{array}$ & $\begin{array}{l}6.81 \\
6.81 \\
7.14 \\
7.11 \\
6.64\end{array}$ & $\begin{array}{l}12.36 \\
12.38 \\
12.76 \\
12.75 \\
12.34\end{array}$ & $\begin{array}{l}4.92 \\
4.91 \\
4.93 \\
4.91 \\
4.93\end{array}$ & $\begin{array}{l}1.23 \\
1.30 \\
1.31 \\
1.31 \\
1.34\end{array}$ & $\begin{array}{l}5.01 \\
4.89 \\
5.00 \\
5.00 \\
4.75\end{array}$ & $\begin{array}{l}11.16 \\
11.10 \\
11.24 \\
11.22 \\
11.02\end{array}$ \\
\hline $\begin{array}{l}1880 \\
1881 \\
1882 \\
1883 \\
1884\end{array}$ & $\begin{array}{l}4.84 \\
4.82 \\
4.86 \\
4.87 \\
4.92\end{array}$ & $\begin{array}{r}.95 \\
.99 \\
1.02 \\
1.36 \\
1.54\end{array}$ & $\begin{array}{l}7.03 \\
6.51 \\
7.32 \\
7.01 \\
6.92\end{array}$ & $\begin{array}{l}12.82 \\
12.32 \\
13.20 \\
13.24 \\
13.39\end{array}$ & $\begin{array}{l}4.95 \\
4.93 \\
4.97 \\
4.98 \\
5.03\end{array}$ & $\begin{array}{l}1.45 \\
1.51 \\
1.53 \\
1.55 \\
1.59\end{array}$ & $\begin{array}{l}4.83 \\
4.66 \\
4.51 \\
4.61 \\
4.44\end{array}$ & $\begin{array}{l}11.22 \\
11.10 \\
11.00 \\
11.14 \\
11.06\end{array}$ \\
\hline $\begin{array}{l}1885 \\
1886 \\
1887 \\
1888 \\
1889\end{array}$ & $\begin{array}{l}4.98 \\
5.04 \\
5.07 \\
5.12 \\
5.14\end{array}$ & $\begin{array}{l}1.50 \\
1.57 \\
1.88 \\
2.09 \\
2.33\end{array}$ & $\begin{array}{l}6.95 \\
7.35 \\
7.52 \\
7.83 \\
8.19\end{array}$ & $\begin{array}{l}13.43 \\
13.95 \\
14.47 \\
15.04 \\
15.67\end{array}$ & $\begin{array}{l}5.08 \\
5.13 \\
5.16 \\
5.22 \\
5.24\end{array}$ & $\begin{array}{l}1.57 \\
1.63 \\
1.65 \\
1.85 \\
1.86\end{array}$ & $\begin{array}{l}4.49 \\
4.84 \\
4.96 \\
5.09 \\
5.27\end{array}$ & $\begin{array}{l}11.14 \\
11.60 \\
11.77 \\
12.16 \\
12.38\end{array}$ \\
\hline $\begin{array}{l}1890 \\
1891 \\
1892 \\
1893 \\
1894\end{array}$ & $\begin{array}{l}5.15 \\
5.16 \\
5.14 \\
5.14 \\
5.16\end{array}$ & $\begin{array}{l}2.36 \\
2.34 \\
2.32 \\
2.44 \\
2.51\end{array}$ & $\begin{array}{l}8.37 \\
8.52 \\
8.17 \\
8.02 \\
7.93\end{array}$ & $\begin{array}{l}15.88 \\
16.02 \\
15.63 \\
15.60 \\
15.60\end{array}$ & $\begin{array}{l}5.25 \\
5.27 \\
5.26 \\
5.28 \\
5.32\end{array}$ & $\begin{array}{l}1.96 \\
1.93 \\
1.93 \\
2.03 \\
2.02\end{array}$ & $\begin{array}{l}5.33 \\
5.34 \\
5.35 \\
5.52 \\
5.43\end{array}$ & $\begin{array}{l}12.54 \\
12.54 \\
12.54 \\
12.83 \\
12.76\end{array}$ \\
\hline $\begin{array}{l}1895 \\
1896 \\
1897 \\
1898 \\
1899\end{array}$ & $\begin{array}{l}5.18 \\
5.20 \\
5.26 \\
5.30 \\
5.31\end{array}$ & $\begin{array}{l}2.52 \\
2.60 \\
2.66 \\
2.70 \\
2.79\end{array}$ & $\begin{array}{l}7.95 \\
8.29 \\
8.87 \\
8.59 \\
8.38\end{array}$ & $\begin{array}{l}15.66 \\
16.09 \\
16.79 \\
16.60 \\
16.48\end{array}$ & $\begin{array}{l}5.34 \\
5.38 \\
5.45 \\
5.50 \\
5.51\end{array}$ & $\begin{array}{l}1.98 \\
2.07 \\
2.12 \\
2.19 \\
2.29\end{array}$ & $\begin{array}{l}5.51 \\
5.59 \\
5.59 \\
5.59 \\
5.50\end{array}$ & $\begin{array}{l}12.84 \\
13.04 \\
13.16 \\
13.28 \\
13.30\end{array}$ \\
\hline $\begin{array}{l}1900 \\
1901 \\
1902 \\
1903 \\
1904\end{array}$ & $\begin{array}{l}5.32 \\
5.38 \\
5.43 \\
5.48 \\
5.55\end{array}$ & $\begin{array}{l}2.84 \\
2.92 \\
3.05 \\
3.18 \\
3.30\end{array}$ & $\begin{array}{l}7.93 \\
8.05 \\
8.44 \\
8.51 \\
8.64\end{array}$ & $\begin{array}{l}16.09 \\
16.35 \\
16.92 \\
17.17 \\
17.49\end{array}$ & $\begin{array}{l}5.53 \\
5.60 \\
5.64 \\
5.67 \\
5.73\end{array}$ & $\begin{array}{l}2.34 \\
2.39 \\
2.52 \\
2.59 \\
2.70\end{array}$ & $\begin{array}{l}5.25 \\
5.28 \\
5.43 \\
5.47 \\
5.57\end{array}$ & $\begin{array}{l}13.12 \\
13.27 \\
13.58 \\
13.73 \\
14.00\end{array}$ \\
\hline $\begin{array}{l}1905 \\
1906 \\
1907 \\
1908 \\
1909\end{array}$ & $\begin{array}{l}5.60 \\
5.63 \\
5.71 \\
5.82 \\
5.97\end{array}$ & $\begin{array}{l}3.34 \\
3.59 \\
3.59 \\
3.80 \\
4.03\end{array}$ & $\begin{array}{l}8.82 \\
8.55 \\
8.60 \\
8.64 \\
8.97\end{array}$ & $\begin{array}{l}17.77 \\
17.77 \\
17.90 \\
18.26 \\
18.97\end{array}$ & $\begin{array}{l}5.77 \\
5.77 \\
5.83 \\
5.92 \\
6.04\end{array}$ & $\begin{array}{l}2.72 \\
2.87 \\
2.88 \\
3.06 \\
3.16\end{array}$ & $\begin{array}{l}5.65 \\
5.44 \\
5.44 \\
5.42 \\
5.56\end{array}$ & $\begin{array}{l}14.14 \\
14.07 \\
14.15 \\
14.41 \\
14.77\end{array}$ \\
\hline $\begin{array}{l}1910 \\
1911 \\
1912 \\
1913\end{array}$ & $\begin{array}{l}6.12 \\
6.36 \\
6.53 \\
6.74\end{array}$ & $\begin{array}{l}4.24 \\
4.48 \\
4.59 \\
4.79\end{array}$ & $\begin{array}{r}9.44 \\
10.14 \\
10.44 \\
10.46\end{array}$ & $\begin{array}{l}19.80 \\
20.98 \\
21.56 \\
21.99\end{array}$ & $\begin{array}{l}6.15 \\
6.32 \\
6.43 \\
6.52\end{array}$ & $\begin{array}{l}3.37 \\
3.51 \\
3.63 \\
3.85\end{array}$ & $\begin{array}{l}5.81 \\
6.19 \\
6.28 \\
6.33\end{array}$ & $\begin{array}{l}15.33 \\
16.03 \\
16.33 \\
16.70\end{array}$ \\
\hline
\end{tabular}




\begin{tabular}{|c|c|c|c|c|c|c|c|c|}
\hline & $(25)$ & \multicolumn{2}{|c|}{ Marches } & $(28)$ & \multicolumn{4}{|c|}{ Umbria } \\
\hline & $\begin{array}{l}\text { Buil- } \\
\text { dings }\end{array}$ & $\begin{array}{l}\text { Rail- } \\
\text { ways }\end{array}$ & $\begin{array}{l}\text { Other } \\
\text { s.o.c. }\end{array}$ & Total & $\begin{array}{l}\text { Buil- } \\
\text { dings }\end{array}$ & $\begin{array}{l}\text { Rail- } \\
\text { ways }\end{array}$ & $\begin{array}{l}\text { Other } \\
\text { s.o.c. }\end{array}$ & Total \\
\hline $\begin{array}{l}1861 \\
1862 \\
1863 \\
1864\end{array}$ & $\begin{array}{l}1.74 \\
1.76 \\
1.77 \\
1.78\end{array}$ & $\begin{array}{l}.06 \\
.06 \\
.14 \\
.15\end{array}$ & $\begin{array}{l}1.25 \\
1.37 \\
1.40 \\
1.45\end{array}$ & $\begin{array}{l}3.05 \\
3.18 \\
3.31 \\
3.37\end{array}$ & $\begin{array}{l}1.03 \\
1.05 \\
1.06 \\
1.07\end{array}$ & $\begin{array}{l}.00 \\
.01 \\
.01 \\
.01\end{array}$ & $\begin{array}{l}.65 \\
.71 \\
.72 \\
.75\end{array}$ & $\begin{array}{l}1.68 \\
1.77 \\
1.79 \\
1.83\end{array}$ \\
\hline $\begin{array}{l}1865 \\
1866 \\
1867 \\
1868 \\
1869\end{array}$ & $\begin{array}{l}1.76 \\
1.74 \\
1.74 \\
1.73 \\
1.72\end{array}$ & $\begin{array}{l}.16 \\
.22 \\
.22 \\
.23 \\
.24\end{array}$ & $\begin{array}{l}1.41 \\
1.51 \\
1.47 \\
1.44 \\
1.52\end{array}$ & $\begin{array}{l}3.33 \\
3.47 \\
3.43 \\
3.40 \\
3.48\end{array}$ & $\begin{array}{l}1.07 \\
1.05 \\
1.06 \\
1.06 \\
1.06\end{array}$ & $\begin{array}{l}.04 \\
.23 \\
.23 \\
.24 \\
.25\end{array}$ & $\begin{array}{l}.79 \\
.83 \\
.81 \\
.82 \\
.88\end{array}$ & $\begin{array}{l}1.90 \\
2.12 \\
2.11 \\
2.12 \\
2.19\end{array}$ \\
\hline $\begin{array}{l}1870 \\
1871 \\
1872 \\
1873 \\
1874\end{array}$ & $\begin{array}{l}1.75 \\
1.73 \\
1.72 \\
1.73 \\
1.72\end{array}$ & $\begin{array}{l}.25 \\
.27 \\
.29 \\
.33 \\
.31\end{array}$ & $\begin{array}{l}1.44 \\
1.41 \\
1.47 \\
1.41 \\
1.50\end{array}$ & $\begin{array}{l}3.44 \\
3.42 \\
3.47 \\
3.47 \\
3.54\end{array}$ & $\begin{array}{l}1.08 \\
1.08 \\
1.07 \\
1.07 \\
1.07\end{array}$ & $\begin{array}{l}.26 \\
.28 \\
.30 \\
.33 \\
.36\end{array}$ & $\begin{array}{l}.92 \\
.96 \\
.96 \\
.92 \\
.97\end{array}$ & $\begin{array}{l}2.26 \\
2.31 \\
2.33 \\
2.33 \\
2.40\end{array}$ \\
\hline $\begin{array}{l}1875 \\
1876 \\
1877 \\
1878 \\
1879\end{array}$ & $\begin{array}{l}1.73 \\
1.72 \\
1.74 \\
1.75 \\
1.76\end{array}$ & $\begin{array}{l}.32 \\
.33 \\
.33 \\
.33 \\
.34\end{array}$ & $\begin{array}{l}1.56 \\
1.54 \\
1.57 \\
1.62 \\
1.56\end{array}$ & $\begin{array}{l}3.61 \\
3.60 \\
3.64 \\
3.70 \\
3.65\end{array}$ & $\begin{array}{l}1.08 \\
1.07 \\
1.08 \\
1.09 \\
1.10\end{array}$ & $\begin{array}{l}.40 \\
.41 \\
.41 \\
.41 \\
.42\end{array}$ & $\begin{array}{l}.96 \\
.95 \\
.97 \\
.99 \\
.91\end{array}$ & $\begin{array}{l}2.43 \\
2.43 \\
2.46 \\
2.49 \\
2.43\end{array}$ \\
\hline $\begin{array}{l}1880 \\
1881 \\
1882 \\
1883 \\
1884\end{array}$ & $\begin{array}{l}1.77 \\
1.77 \\
1.78 \\
1.79 \\
1.81\end{array}$ & $\begin{array}{l}.37 \\
.38 \\
.38 \\
.39 \\
.45\end{array}$ & $\begin{array}{l}1.54 \\
1.67 \\
1.67 \\
1.65 \\
1.67\end{array}$ & $\begin{array}{l}3.67 \\
3.81 \\
3.83 \\
3.82 \\
3.93\end{array}$ & $\begin{array}{l}1.10 \\
1.10 \\
1.11 \\
1.11 \\
1.12\end{array}$ & $\begin{array}{l}.45 \\
.47 \\
.48 \\
.60 \\
.62\end{array}$ & $\begin{array}{l}.93 \\
.89 \\
.88 \\
.90 \\
.90\end{array}$ & $\begin{array}{l}2.48 \\
2.47 \\
2.47 \\
2.61 \\
2.65\end{array}$ \\
\hline $\begin{array}{l}1885 \\
1886 \\
1887 \\
1888 \\
1889\end{array}$ & $\begin{array}{l}1.83 \\
1.84 \\
1.86 \\
1.88 \\
1.89\end{array}$ & $\begin{array}{l}.45 \\
.51 \\
.51 \\
.57 \\
.57\end{array}$ & $\begin{array}{l}1.70 \\
1.74 \\
1.74 \\
1.78 \\
1.85\end{array}$ & $\begin{array}{l}3.98 \\
4.09 \\
4.11 \\
4.23 \\
4.31\end{array}$ & $\begin{array}{l}1.14 \\
1.15 \\
1.17 \\
1.19 \\
1.21\end{array}$ & $\begin{array}{l}.60 \\
.71 \\
.72 \\
.74 \\
.74\end{array}$ & $\begin{array}{r}.95 \\
1.00 \\
1.04 \\
1.07 \\
1.09\end{array}$ & $\begin{array}{l}2.69 \\
2.86 \\
2.93 \\
3.00 \\
3.03\end{array}$ \\
\hline $\begin{array}{l}1890 \\
1891 \\
1892 \\
1893 \\
1894\end{array}$ & $\begin{array}{l}1.89 \\
1.90 \\
1.89 \\
1.90 \\
1.91\end{array}$ & $\begin{array}{l}.57 \\
.56 \\
.54 \\
.55 \\
.54\end{array}$ & $\begin{array}{l}1.84 \\
1.90 \\
1.98 \\
2.06 \\
2.03\end{array}$ & $\begin{array}{l}4.30 \\
4.36 \\
4.41 \\
4.51 \\
4.48\end{array}$ & $\begin{array}{l}1.21 \\
1.21 \\
1.21 \\
1.22 \\
1.22\end{array}$ & $\begin{array}{l}.74 \\
.72 \\
.70 \\
.71 \\
.70\end{array}$ & $\begin{array}{l}1.08 \\
1.11 \\
1.13 \\
1.15 \\
1.14\end{array}$ & $\begin{array}{l}3.03 \\
3.05 \\
3.04 \\
3.08 \\
3.06\end{array}$ \\
\hline $\begin{array}{l}1895 \\
1896 \\
1897 \\
1898 \\
1899\end{array}$ & $\begin{array}{l}1.93 \\
1.94 \\
1.95 \\
1.96 \\
1.96\end{array}$ & $\begin{array}{l}.58 \\
.59 \\
.61 \\
.69 \\
.72\end{array}$ & $\begin{array}{l}1.98 \\
2.04 \\
2.13 \\
2.12 \\
2.12\end{array}$ & $\begin{array}{l}4.49 \\
4.57 \\
4.70 \\
4.77 \\
4.80\end{array}$ & $\begin{array}{l}1.23 \\
1.23 \\
1.24 \\
1.25 \\
1.25\end{array}$ & $\begin{array}{l}.69 \\
.71 \\
.73 \\
.74 \\
.77\end{array}$ & $\begin{array}{l}1.13 \\
1.16 \\
1.18 \\
1.17 \\
1.15\end{array}$ & $\begin{array}{l}3.05 \\
3.11 \\
3.15 \\
3.16 \\
3.16\end{array}$ \\
\hline $\begin{array}{l}1900 \\
1901 \\
1902 \\
1903 \\
1904\end{array}$ & $\begin{array}{l}1.97 \\
1.99 \\
1.99 \\
2.00 \\
2.01\end{array}$ & $\begin{array}{l}.73 \\
.75 \\
.77 \\
.80 \\
.83\end{array}$ & $\begin{array}{l}2.07 \\
2.04 \\
2.07 \\
2.12 \\
2.18\end{array}$ & $\begin{array}{l}4.77 \\
4.77 \\
4.83 \\
4.92 \\
5.02\end{array}$ & $\begin{array}{l}1.25 \\
1.26 \\
1.27 \\
1.28 \\
1.29\end{array}$ & $\begin{array}{l}.79 \\
.81 \\
.84 \\
.86 \\
.90\end{array}$ & $\begin{array}{l}1.11 \\
1.11 \\
1.12 \\
1.13 \\
1.14\end{array}$ & $\begin{array}{l}3.14 \\
3.18 \\
3.23 \\
3.26 \\
3.33\end{array}$ \\
\hline $\begin{array}{l}1905 \\
1906 \\
1907 \\
1908 \\
1909\end{array}$ & $\begin{array}{l}2.02 \\
2.02 \\
2.04 \\
2.06 \\
2.10\end{array}$ & $\begin{array}{r}.84 \\
.92 \\
.92 \\
1.09 \\
1.13\end{array}$ & $\begin{array}{l}2.22 \\
2.15 \\
2.15 \\
2.12 \\
2.20\end{array}$ & $\begin{array}{l}5.08 \\
5.09 \\
5.11 \\
5.28 \\
5.42\end{array}$ & $\begin{array}{l}1.31 \\
1.32 \\
1.33 \\
1.34 \\
1.35\end{array}$ & $\begin{array}{r}.91 \\
.96 \\
.96 \\
1.02 \\
1.05\end{array}$ & $\begin{array}{l}1.15 \\
1.11 \\
1.11 \\
1.12 \\
1.15\end{array}$ & $\begin{array}{l}3.37 \\
3.38 \\
3.40 \\
3.47 \\
3.56\end{array}$ \\
\hline $\begin{array}{l}1910 \\
1911 \\
1912 \\
1913\end{array}$ & $\begin{array}{l}2.12 \\
2.18 \\
2.20 \\
2.23\end{array}$ & $\begin{array}{l}1.18 \\
1.20 \\
1.23 \\
1.26\end{array}$ & $\begin{array}{l}2.32 \\
2.47 \\
2.50 \\
2.57\end{array}$ & $\begin{array}{l}5.62 \\
5.84 \\
5.93 \\
6.06\end{array}$ & $\begin{array}{l}1.37 \\
1.41 \\
1.43 \\
1.45\end{array}$ & $\begin{array}{l}1.10 \\
1.12 \\
1.14 \\
1.17\end{array}$ & $\begin{array}{l}1.19 \\
1.27 \\
1.30 \\
1.31\end{array}$ & $\begin{array}{l}3.66 \\
3.80 \\
3.86 \\
3.93\end{array}$ \\
\hline
\end{tabular}




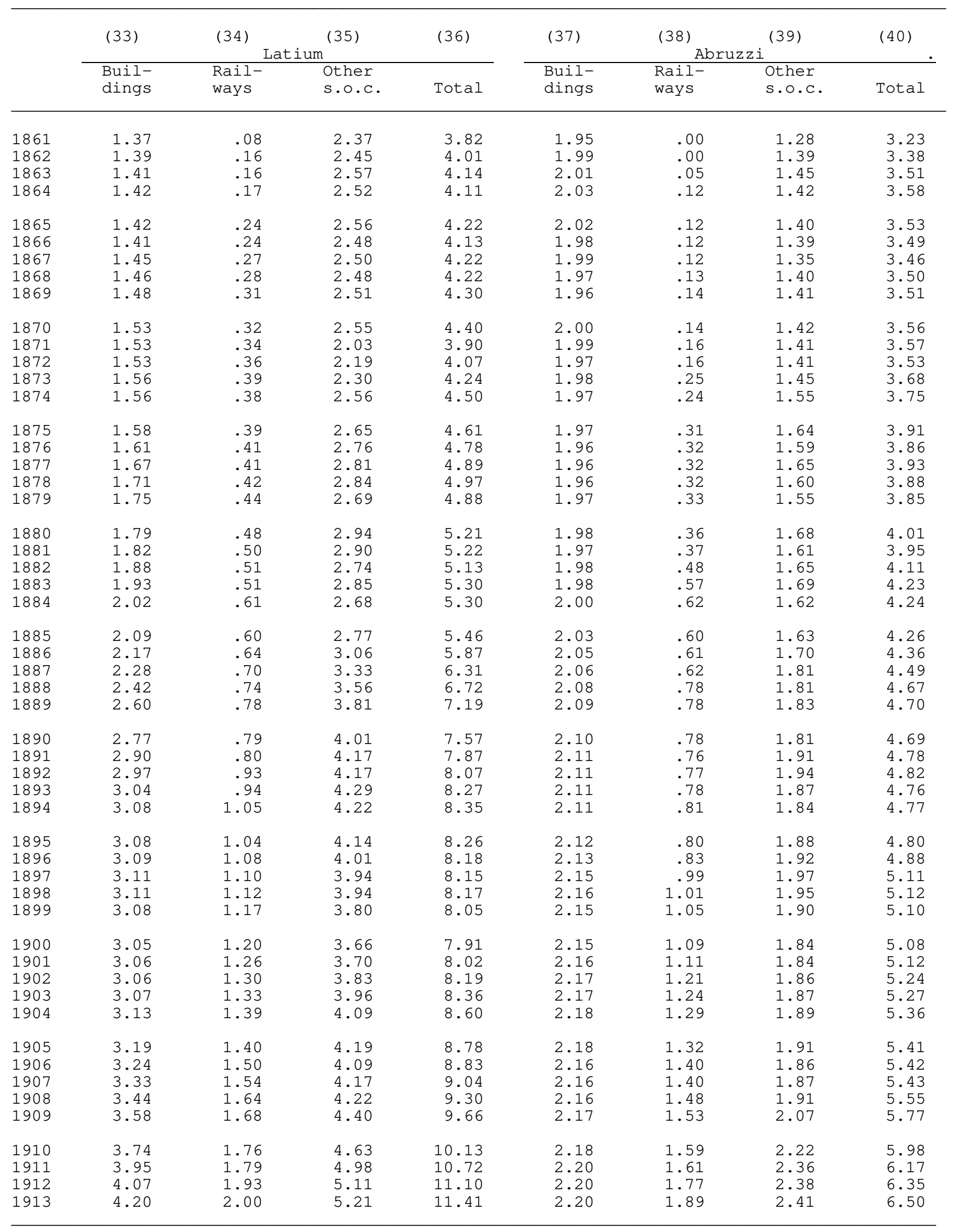




\begin{tabular}{|c|c|c|c|c|c|c|c|c|}
\hline & $(41)$ & \multicolumn{2}{|c|}{ Campania } & $(44)$ & $(45)$ & $\begin{array}{ll}(46) & \\
& A\end{array}$ & $a^{(47)}$ & $(48)$ \\
\hline & $\begin{array}{l}\text { Buil- } \\
\text { dings }\end{array}$ & $\begin{array}{l}\text { Rail- } \\
\text { ways }\end{array}$ & $\begin{array}{l}\text { Other } \\
\text { s.o.c. }\end{array}$ & Total & $\begin{array}{l}\text { Buil- } \\
\text { dings }\end{array}$ & $\begin{array}{l}\text { Rail- } \\
\text { ways }\end{array}$ & $\begin{array}{l}\text { Other } \\
\text { s.o.c. }\end{array}$ & Total \\
\hline $\begin{array}{l}1861 \\
1862 \\
1863 \\
1864\end{array}$ & $\begin{array}{l}5.25 \\
5.35 \\
5.39 \\
5.44\end{array}$ & $\begin{array}{l}.12 \\
.12 \\
.16 \\
.17\end{array}$ & $\begin{array}{l}2.52 \\
2.75 \\
2.72 \\
2.81\end{array}$ & $\begin{array}{l}7.89 \\
8.22 \\
8.27 \\
8.42\end{array}$ & $\begin{array}{l}3.32 \\
3.39 \\
3.41 \\
3.44\end{array}$ & $\begin{array}{l}.00 \\
.00 \\
.00 \\
.08\end{array}$ & $\begin{array}{l}1.46 \\
1.58 \\
1.65 \\
1.67\end{array}$ & $\begin{array}{l}4.77 \\
4.97 \\
5.06 \\
5.20\end{array}$ \\
\hline $\begin{array}{l}1865 \\
1866 \\
1867 \\
1868 \\
1869\end{array}$ & $\begin{array}{l}5.40 \\
5.28 \\
5.33 \\
5.30 \\
5.29\end{array}$ & $\begin{array}{l}.17 \\
.18 \\
.20 \\
.28 \\
.32\end{array}$ & $\begin{array}{l}3.01 \\
3.44 \\
3.50 \\
3.61 \\
3.74\end{array}$ & $\begin{array}{l}8.57 \\
8.90 \\
9.03 \\
9.19 \\
9.35\end{array}$ & $\begin{array}{l}3.43 \\
3.39 \\
3.45 \\
3.47 \\
3.49\end{array}$ & $\begin{array}{l}.19 \\
.21 \\
.23 \\
.31 \\
.35\end{array}$ & $\begin{array}{l}1.72 \\
2.09 \\
2.17 \\
2.25 \\
2.37\end{array}$ & $\begin{array}{l}5.34 \\
5.70 \\
5.85 \\
6.03 \\
6.21\end{array}$ \\
\hline $\begin{array}{l}1870 \\
1871 \\
1872 \\
1873 \\
1874\end{array}$ & $\begin{array}{l}5.43 \\
5.39 \\
5.34 \\
5.41 \\
5.40\end{array}$ & $\begin{array}{l}.34 \\
.36 \\
.38 \\
.41 \\
.41\end{array}$ & $\begin{array}{l}3.88 \\
3.84 \\
3.75 \\
3.72 \\
3.76\end{array}$ & $\begin{array}{l}9.65 \\
9.60 \\
9.47 \\
9.54 \\
9.56\end{array}$ & $\begin{array}{l}3.60 \\
3.60 \\
3.57 \\
3.64 \\
3.64\end{array}$ & $\begin{array}{l}.37 \\
.40 \\
.43 \\
.47 \\
.45\end{array}$ & $\begin{array}{l}2.38 \\
2.69 \\
2.36 \\
2.37 \\
2.58\end{array}$ & $\begin{array}{l}6.35 \\
6.69 \\
6.36 \\
6.48 \\
6.67\end{array}$ \\
\hline $\begin{array}{l}1875 \\
1876 \\
1877 \\
1878 \\
1879\end{array}$ & $\begin{array}{l}5.41 \\
5.40 \\
5.45 \\
5.45 \\
5.50\end{array}$ & $\begin{array}{l}.44 \\
.46 \\
.47 \\
.47 \\
.51\end{array}$ & $\begin{array}{l}3.71 \\
3.86 \\
4.03 \\
4.19 \\
4.05\end{array}$ & $\begin{array}{r}9.56 \\
9.72 \\
9.95 \\
10.11 \\
10.06\end{array}$ & $\begin{array}{l}3.67 \\
3.68 \\
3.74 \\
3.75 \\
3.80\end{array}$ & $\begin{array}{l}.45 \\
.47 \\
.47 \\
.47 \\
.48\end{array}$ & $\begin{array}{l}2.54 \\
2.68 \\
2.61 \\
2.72 \\
2.60\end{array}$ & $\begin{array}{l}6.66 \\
6.83 \\
6.82 \\
6.94 \\
6.88\end{array}$ \\
\hline $\begin{array}{l}1880 \\
1881 \\
1882 \\
1883 \\
1884\end{array}$ & $\begin{array}{l}5.53 \\
5.50 \\
5.54 \\
5.55 \\
5.60\end{array}$ & $\begin{array}{l}.55 \\
.58 \\
.64 \\
.70 \\
.73\end{array}$ & $\begin{array}{l}4.31 \\
4.23 \\
4.07 \\
3.92 \\
4.05\end{array}$ & $\begin{array}{l}10.38 \\
10.31 \\
10.26 \\
10.17 \\
10.38\end{array}$ & $\begin{array}{l}3.84 \\
3.84 \\
3.87 \\
3.89 \\
3.94\end{array}$ & $\begin{array}{l}.52 \\
.54 \\
.55 \\
.59 \\
.64\end{array}$ & $\begin{array}{l}2.66 \\
2.72 \\
2.71 \\
2.69 \\
2.74\end{array}$ & $\begin{array}{l}7.02 \\
7.09 \\
7.13 \\
7.17 \\
7.32\end{array}$ \\
\hline $\begin{array}{l}1885 \\
1886 \\
1887 \\
1888 \\
1889\end{array}$ & $\begin{array}{l}5.65 \\
5.71 \\
5.76 \\
5.81 \\
5.82\end{array}$ & $\begin{array}{l}.78 \\
.84 \\
.88 \\
.93 \\
.96\end{array}$ & $\begin{array}{l}4.39 \\
4.41 \\
4.46 \\
4.65 \\
4.80\end{array}$ & $\begin{array}{l}10.82 \\
10.95 \\
11.10 \\
11.39 \\
11.58\end{array}$ & $\begin{array}{l}3.99 \\
4.05 \\
4.12 \\
4.21 \\
4.26\end{array}$ & $\begin{array}{l}.67 \\
.72 \\
.74 \\
.76 \\
.77\end{array}$ & $\begin{array}{l}2.82 \\
2.97 \\
3.18 \\
3.38 \\
3.43\end{array}$ & $\begin{array}{l}7.48 \\
7.74 \\
8.05 \\
8.34 \\
8.46\end{array}$ \\
\hline $\begin{array}{l}1890 \\
1891 \\
1892 \\
1893 \\
1894\end{array}$ & $\begin{array}{l}5.82 \\
5.86 \\
5.88 \\
5.91 \\
5.95\end{array}$ & $\begin{array}{r}.97 \\
1.00 \\
1.06 \\
1.11 \\
1.14\end{array}$ & $\begin{array}{l}4.83 \\
5.01 \\
5.02 \\
4.95 \\
4.84\end{array}$ & $\begin{array}{l}11.62 \\
11.88 \\
11.96 \\
11.97 \\
11.93\end{array}$ & $\begin{array}{l}4.28 \\
4.30 \\
4.27 \\
4.27 \\
4.27\end{array}$ & $\begin{array}{l}.76 \\
.76 \\
.81 \\
.82 \\
.81\end{array}$ & $\begin{array}{l}3.41 \\
3.55 \\
3.58 \\
3.58 \\
3.55\end{array}$ & $\begin{array}{l}8.45 \\
8.61 \\
8.66 \\
8.66 \\
8.64\end{array}$ \\
\hline $\begin{array}{l}1895 \\
1896 \\
1897 \\
1898 \\
1899\end{array}$ & $\begin{array}{l}6.01 \\
6.10 \\
6.22 \\
6.29 \\
6.28\end{array}$ & $\begin{array}{l}1.21 \\
1.25 \\
1.28 \\
1.30 \\
1.36\end{array}$ & $\begin{array}{l}4.85 \\
5.00 \\
4.99 \\
5.05 \\
5.07\end{array}$ & $\begin{array}{l}12.08 \\
12.35 \\
12.50 \\
12.64 \\
12.72\end{array}$ & $\begin{array}{l}4.28 \\
4.31 \\
4.36 \\
4.41 \\
4.43\end{array}$ & $\begin{array}{l}.86 \\
.89 \\
.91 \\
.92 \\
.96\end{array}$ & $\begin{array}{l}3.71 \\
3.85 \\
3.77 \\
3.75 \\
3.74\end{array}$ & $\begin{array}{l}8.85 \\
9.05 \\
9.05 \\
9.08 \\
9.13\end{array}$ \\
\hline $\begin{array}{l}1900 \\
1901 \\
1902 \\
1903 \\
1904\end{array}$ & $\begin{array}{l}6.26 \\
6.30 \\
6.31 \\
6.30 \\
6.34\end{array}$ & $\begin{array}{l}1.40 \\
1.44 \\
1.52 \\
1.59 \\
1.74\end{array}$ & $\begin{array}{l}4.88 \\
4.82 \\
4.86 \\
4.90 \\
4.98\end{array}$ & $\begin{array}{l}12.55 \\
12.56 \\
12.69 \\
12.79 \\
13.06\end{array}$ & $\begin{array}{l}4.43 \\
4.47 \\
4.51 \\
4.54 \\
4.60\end{array}$ & $\begin{array}{l}1.02 \\
1.04 \\
1.08 \\
1.13 \\
1.18\end{array}$ & $\begin{array}{l}3.61 \\
3.55 \\
3.58 \\
3.63 \\
3.70\end{array}$ & $\begin{array}{l}9.06 \\
9.06 \\
9.17 \\
9.30 \\
9.48\end{array}$ \\
\hline $\begin{array}{l}1905 \\
1906 \\
1907 \\
1908 \\
1909\end{array}$ & $\begin{array}{l}6.35 \\
6.28 \\
6.28 \\
6.28 \\
6.32\end{array}$ & $\begin{array}{l}1.78 \\
1.87 \\
1.88 \\
1.98 \\
2.04\end{array}$ & $\begin{array}{l}4.99 \\
4.82 \\
4.82 \\
4.84 \\
4.93\end{array}$ & $\begin{array}{l}13.12 \\
12.97 \\
12.98 \\
13.11 \\
13.28\end{array}$ & $\begin{array}{l}4.63 \\
4.59 \\
4.59 \\
4.61 \\
4.65\end{array}$ & $\begin{array}{l}1.24 \\
1.31 \\
1.41 \\
1.50 \\
1.54\end{array}$ & $\begin{array}{l}3.73 \\
3.58 \\
3.55 \\
3.49 \\
3.56\end{array}$ & $\begin{array}{l}9.60 \\
9.48 \\
9.56 \\
9.60 \\
9.75\end{array}$ \\
\hline $\begin{array}{l}1910 \\
1911 \\
1912 \\
1913\end{array}$ & $\begin{array}{l}6.35 \\
6.46 \\
6.48 \\
6.53\end{array}$ & $\begin{array}{l}2.19 \\
2.25 \\
2.30 \\
2.48\end{array}$ & $\begin{array}{l}5.11 \\
5.44 \\
5.47 \\
5.59\end{array}$ & $\begin{array}{l}13.66 \\
14.15 \\
14.26 \\
14.60\end{array}$ & $\begin{array}{l}4.71 \\
4.82 \\
4.85 \\
4.89\end{array}$ & $\begin{array}{l}1.61 \\
1.75 \\
1.79 \\
1.84\end{array}$ & $\begin{array}{l}3.72 \\
3.95 \\
4.12 \\
4.45\end{array}$ & $\begin{array}{l}10.04 \\
10.52 \\
10.76 \\
11.18\end{array}$ \\
\hline
\end{tabular}




\begin{tabular}{|c|c|c|c|c|c|c|c|c|}
\hline & \multirow{2}{*}{$\begin{array}{l}\text { (49) } \\
\begin{array}{l}\text { Buil- } \\
\text { dings }\end{array}\end{array}$} & $\begin{array}{l}(50) \\
\mathrm{Ba}\end{array}$ & $\begin{array}{l}(51) \\
\text { ata }\end{array}$ & \multirow{2}{*}{$\frac{(52)}{\text { Total }}$} & \multirow{2}{*}{$\begin{array}{l}\text { (53) } \\
\begin{array}{l}\text { Buil- } \\
\text { dings }\end{array}\end{array}$} & \multirow{2}{*}{\begin{tabular}{l}
\multicolumn{1}{c}{$\mathrm{Ca}$ Ca } \\
Rail- \\
ways
\end{tabular}} & \multirow{2}{*}{$\begin{array}{l}(55) \\
-a \\
\text { Other } \\
\text { s.o.c. }\end{array}$} & \multirow{2}{*}{$\frac{(56)}{\text { Total }}$} \\
\hline & & $\begin{array}{l}\text { Rail- } \\
\text { ways }\end{array}$ & $\begin{array}{l}\text { Other } \\
\text { s.o.c. }\end{array}$ & & & & & \\
\hline $\begin{array}{l}1861 \\
1862 \\
1863 \\
1864\end{array}$ & $\begin{array}{l}.84 \\
.86 \\
.86 \\
.87\end{array}$ & $\begin{array}{l}.00 \\
.00 \\
.00 \\
.00\end{array}$ & $\begin{array}{l}.57 \\
.62 \\
.64 \\
.61\end{array}$ & $\begin{array}{l}1.41 \\
1.47 \\
1.51 \\
1.48\end{array}$ & $\begin{array}{l}1.38 \\
1.41 \\
1.42 \\
1.43\end{array}$ & $\begin{array}{l}.00 \\
.00 \\
.00 \\
.00\end{array}$ & $\begin{array}{r}.89 \\
.97 \\
1.02 \\
1.04\end{array}$ & $\begin{array}{l}2.27 \\
2.38 \\
2.43 \\
2.47\end{array}$ \\
\hline $\begin{array}{l}1865 \\
1866 \\
1867 \\
1868 \\
1869\end{array}$ & $\begin{array}{l}.86 \\
.84 \\
.85 \\
.84 \\
.84\end{array}$ & $\begin{array}{l}.00 \\
.00 \\
.00 \\
.00 \\
.02\end{array}$ & $\begin{array}{l}.60 \\
.58 \\
.54 \\
.55 \\
.63\end{array}$ & $\begin{array}{l}1.46 \\
1.42 \\
1.39 \\
1.39 \\
1.48\end{array}$ & $\begin{array}{l}1.42 \\
1.40 \\
1.42 \\
1.42 \\
1.42\end{array}$ & $\begin{array}{l}.00 \\
.01 \\
.01 \\
.04 \\
.07\end{array}$ & $\begin{array}{l}1.04 \\
1.19 \\
1.16 \\
1.18 \\
1.36\end{array}$ & $\begin{array}{l}2.46 \\
2.61 \\
2.59 \\
2.63 \\
2.85\end{array}$ \\
\hline $\begin{array}{l}1870 \\
1871 \\
1872 \\
1873 \\
1874\end{array}$ & $\begin{array}{l}.86 \\
.85 \\
.84 \\
.85 \\
.85\end{array}$ & $\begin{array}{l}.02 \\
.03 \\
.03 \\
.03 \\
.03\end{array}$ & $\begin{array}{l}.64 \\
.66 \\
.67 \\
.67 \\
.69\end{array}$ & $\begin{array}{l}1.52 \\
1.54 \\
1.54 \\
1.55 \\
1.57\end{array}$ & $\begin{array}{l}1.46 \\
1.45 \\
1.44 \\
1.47 \\
1.47\end{array}$ & $\begin{array}{l}.11 \\
.15 \\
.17 \\
.19 \\
.22\end{array}$ & $\begin{array}{l}1.39 \\
1.34 \\
1.32 \\
1.33 \\
1.40\end{array}$ & $\begin{array}{l}2.95 \\
2.94 \\
2.93 \\
2.98 \\
3.09\end{array}$ \\
\hline $\begin{array}{l}1875 \\
1876 \\
1877 \\
1878 \\
1879\end{array}$ & $\begin{array}{l}.85 \\
.84 \\
.85 \\
.86 \\
.87\end{array}$ & $\begin{array}{l}.06 \\
.08 \\
.09 \\
.09 \\
.10\end{array}$ & $\begin{array}{l}.72 \\
.77 \\
.75 \\
.91 \\
.87\end{array}$ & $\begin{array}{l}1.63 \\
1.70 \\
1.69 \\
1.86 \\
1.84\end{array}$ & $\begin{array}{l}1.48 \\
1.49 \\
1.50 \\
1.51 \\
1.53\end{array}$ & $\begin{array}{l}.30 \\
.33 \\
.36 \\
.36 \\
.38\end{array}$ & $\begin{array}{l}1.39 \\
1.55 \\
1.51 \\
1.55 \\
1.52\end{array}$ & $\begin{array}{l}3.17 \\
3.36 \\
3.38 \\
3.42 \\
3.43\end{array}$ \\
\hline $\begin{array}{l}1880 \\
1881 \\
1882 \\
1883 \\
1884\end{array}$ & $\begin{array}{l}.87 \\
.87 \\
.87 \\
.87 \\
.87\end{array}$ & $\begin{array}{l}.17 \\
.17 \\
.18 \\
.18 \\
.18\end{array}$ & $\begin{array}{r}.83 \\
.93 \\
.90 \\
.92 \\
1.02\end{array}$ & $\begin{array}{l}1.87 \\
1.96 \\
1.95 \\
1.97 \\
2.07\end{array}$ & $\begin{array}{l}1.54 \\
1.54 \\
1.54 \\
1.54 \\
1.56\end{array}$ & $\begin{array}{l}.41 \\
.42 \\
.43 \\
.45 \\
.47\end{array}$ & $\begin{array}{l}1.54 \\
1.56 \\
1.51 \\
1.61 \\
1.57\end{array}$ & $\begin{array}{l}3.49 \\
3.52 \\
3.49 \\
3.60 \\
3.60\end{array}$ \\
\hline $\begin{array}{l}1885 \\
1886 \\
1887 \\
1888 \\
1889\end{array}$ & $\begin{array}{l}.87 \\
.88 \\
.88 \\
.89 \\
.89\end{array}$ & $\begin{array}{l}.18 \\
.17 \\
.18 \\
.18 \\
.18\end{array}$ & $\begin{array}{l}1.11 \\
1.11 \\
1.08 \\
1.03 \\
1.01\end{array}$ & $\begin{array}{l}2.16 \\
2.16 \\
2.14 \\
2.10 \\
2.08\end{array}$ & $\begin{array}{l}1.57 \\
1.59 \\
1.60 \\
1.62 \\
1.63\end{array}$ & $\begin{array}{l}.46 \\
.46 \\
.47 \\
.49 \\
.50\end{array}$ & $\begin{array}{l}1.67 \\
1.72 \\
1.76 \\
1.81 \\
1.82\end{array}$ & $\begin{array}{l}3.71 \\
3.77 \\
3.83 \\
3.92 \\
3.95\end{array}$ \\
\hline $\begin{array}{l}1890 \\
1891 \\
1892 \\
1893 \\
1894\end{array}$ & $\begin{array}{l}.89 \\
.89 \\
.88 \\
.87 \\
.87\end{array}$ & $\begin{array}{l}.18 \\
.19 \\
.24 \\
.24 \\
.25\end{array}$ & $\begin{array}{l}1.04 \\
1.07 \\
1.11 \\
1.12 \\
1.10\end{array}$ & $\begin{array}{l}2.10 \\
2.15 \\
2.23 \\
2.24 \\
2.22\end{array}$ & $\begin{array}{l}1.63 \\
1.64 \\
1.63 \\
1.63 \\
1.63\end{array}$ & $\begin{array}{l}.50 \\
.50 \\
.49 \\
.51 \\
.59\end{array}$ & $\begin{array}{l}1.79 \\
1.87 \\
1.95 \\
1.99 \\
1.96\end{array}$ & $\begin{array}{l}3.92 \\
4.00 \\
4.07 \\
4.13 \\
4.18\end{array}$ \\
\hline $\begin{array}{l}1895 \\
1896 \\
1897 \\
1898 \\
1899\end{array}$ & $\begin{array}{l}.86 \\
.86 \\
.87 \\
.86 \\
.86\end{array}$ & $\begin{array}{l}.25 \\
.25 \\
.30 \\
.31 \\
.32\end{array}$ & $\begin{array}{l}1.12 \\
1.13 \\
1.14 \\
1.13 \\
1.11\end{array}$ & $\begin{array}{l}2.23 \\
2.24 \\
2.31 \\
2.31 \\
2.29\end{array}$ & $\begin{array}{l}1.63 \\
1.64 \\
1.65 \\
1.66 \\
1.66\end{array}$ & $\begin{array}{l}.68 \\
.71 \\
.73 \\
.74 \\
.78\end{array}$ & $\begin{array}{l}1.98 \\
2.02 \\
2.07 \\
2.09 \\
2.09\end{array}$ & $\begin{array}{l}4.29 \\
4.36 \\
4.45 \\
4.48 \\
4.52\end{array}$ \\
\hline $\begin{array}{l}1900 \\
1901 \\
1902 \\
1903 \\
1904\end{array}$ & $\begin{array}{l}.85 \\
.86 \\
.86 \\
.85 \\
.86\end{array}$ & $\begin{array}{l}.33 \\
.33 \\
.34 \\
.35 \\
.37\end{array}$ & $\begin{array}{l}1.07 \\
1.06 \\
1.06 \\
1.05 \\
1.05\end{array}$ & $\begin{array}{l}2.25 \\
2.25 \\
2.26 \\
2.25 \\
2.27\end{array}$ & $\begin{array}{l}1.66 \\
1.67 \\
1.69 \\
1.71 \\
1.73\end{array}$ & $\begin{array}{l}.79 \\
.81 \\
.84 \\
.86 \\
.90\end{array}$ & $\begin{array}{l}2.02 \\
2.03 \\
2.05 \\
2.06 \\
2.10\end{array}$ & $\begin{array}{l}4.47 \\
4.51 \\
4.58 \\
4.63 \\
4.73\end{array}$ \\
\hline $\begin{array}{l}1905 \\
1906 \\
1907 \\
1908 \\
1909\end{array}$ & $\begin{array}{l}.85 \\
.85 \\
.85 \\
.85 \\
.85\end{array}$ & $\begin{array}{l}.37 \\
.39 \\
.39 \\
.42 \\
.43\end{array}$ & $\begin{array}{r}1.04 \\
.98 \\
1.00 \\
1.03 \\
1.11\end{array}$ & $\begin{array}{l}2.26 \\
2.21 \\
2.24 \\
2.31 \\
2.39\end{array}$ & $\begin{array}{l}1.73 \\
1.72 \\
1.72 \\
1.74 \\
1.52\end{array}$ & $\begin{array}{r}.91 \\
.96 \\
.96 \\
1.02 \\
1.05\end{array}$ & $\begin{array}{l}2.11 \\
2.02 \\
2.01 \\
1.97 \\
1.98\end{array}$ & $\begin{array}{l}4.75 \\
4.70 \\
4.69 \\
4.72 \\
4.54\end{array}$ \\
\hline $\begin{array}{l}1910 \\
1911 \\
1912 \\
1913\end{array}$ & $\begin{array}{l}.86 \\
.86 \\
.86 \\
.86\end{array}$ & $\begin{array}{l}.45 \\
.45 \\
.46 \\
.48\end{array}$ & $\begin{array}{l}1.22 \\
1.37 \\
1.43 \\
1.44\end{array}$ & $\begin{array}{l}2.53 \\
2.68 \\
2.75 \\
2.78\end{array}$ & $\begin{array}{l}1.53 \\
1.55 \\
1.55 \\
1.57\end{array}$ & $\begin{array}{l}1.09 \\
1.12 \\
1.15 \\
1.18\end{array}$ & $\begin{array}{l}2.16 \\
2.29 \\
2.30 \\
2.33\end{array}$ & $\begin{array}{l}4.78 \\
4.96 \\
5.00 \\
5.07\end{array}$ \\
\hline
\end{tabular}




\begin{tabular}{|c|c|c|c|c|c|c|c|c|}
\hline & $(57)$ & \multicolumn{2}{|c|}{ Sicily } & $(60)$ & \multicolumn{4}{|c|}{ Sardinia } \\
\hline & $\begin{array}{l}\text { Buil- } \\
\text { dings }\end{array}$ & $\begin{array}{l}\text { Rail- } \\
\text { ways }\end{array}$ & $\begin{array}{l}\text { Other } \\
\text { s.o.c. }\end{array}$ & Total & $\begin{array}{l}\text { Buil- } \\
\text { dings }\end{array}$ & $\begin{array}{l}\text { Rail- } \\
\text { ways }\end{array}$ & $\begin{array}{l}\text { Other } \\
\text { s.o.c. }\end{array}$ & Total \\
\hline $\begin{array}{l}1861 \\
1862 \\
1863 \\
1864\end{array}$ & $\begin{array}{l}3.77 \\
3.85 \\
3.87 \\
3.90\end{array}$ & $\begin{array}{l}.00 \\
.00 \\
.01 \\
.01\end{array}$ & $\begin{array}{l}3.82 \\
4.18 \\
4.45 \\
4.46\end{array}$ & $\begin{array}{l}7.59 \\
8.02 \\
8.33 \\
8.37\end{array}$ & $\begin{array}{l}.89 \\
.91 \\
.92 \\
.92\end{array}$ & $\begin{array}{l}.00 \\
.00 \\
.00 \\
.00\end{array}$ & $\begin{array}{l}1.23 \\
1.33 \\
1.36 \\
1.43\end{array}$ & $\begin{array}{l}2.12 \\
2.24 \\
2.27 \\
2.35\end{array}$ \\
\hline $\begin{array}{l}1865 \\
1866 \\
1867 \\
1868 \\
1869\end{array}$ & $\begin{array}{l}3.90 \\
3.87 \\
3.95 \\
3.98 \\
4.02\end{array}$ & $\begin{array}{l}.01 \\
.04 \\
.06 \\
.06 \\
.09\end{array}$ & $\begin{array}{l}4.65 \\
5.24 \\
5.29 \\
5.16 \\
5.24\end{array}$ & $\begin{array}{l}8.56 \\
9.14 \\
9.30 \\
9.20 \\
9.35\end{array}$ & $\begin{array}{l}.92 \\
.91 \\
.93 \\
.93 \\
.94\end{array}$ & $\begin{array}{l}.00 \\
.00 \\
.00 \\
.00 \\
.00\end{array}$ & $\begin{array}{l}1.47 \\
1.50 \\
1.60 \\
1.53 \\
1.55\end{array}$ & $\begin{array}{l}2.39 \\
2.41 \\
2.52 \\
2.47 \\
2.49\end{array}$ \\
\hline $\begin{array}{l}1870 \\
1871 \\
1872 \\
1873 \\
1874\end{array}$ & $\begin{array}{l}4.15 \\
4.15 \\
4.14 \\
4.23 \\
4.26\end{array}$ & $\begin{array}{l}.13 \\
.18 \\
.19 \\
.20 \\
.22\end{array}$ & $\begin{array}{l}4.99 \\
4.92 \\
4.83 \\
4.64 \\
4.62\end{array}$ & $\begin{array}{l}9.27 \\
9.25 \\
9.16 \\
9.07 \\
9.09\end{array}$ & $\begin{array}{l}.97 \\
.97 \\
.96 \\
.98 \\
.98\end{array}$ & $\begin{array}{l}.00 \\
.01 \\
.03 \\
.03 \\
.04\end{array}$ & $\begin{array}{l}1.49 \\
1.68 \\
1.72 \\
1.62 \\
1.83\end{array}$ & $\begin{array}{l}2.46 \\
2.65 \\
2.71 \\
2.63 \\
2.85\end{array}$ \\
\hline $\begin{array}{l}1875 \\
1876 \\
1877 \\
1878 \\
1879\end{array}$ & $\begin{array}{l}4.30 \\
4.33 \\
4.43 \\
4.47 \\
4.54\end{array}$ & $\begin{array}{l}.23 \\
.30 \\
.30 \\
.30 \\
.31\end{array}$ & $\begin{array}{l}4.59 \\
4.64 \\
4.75 \\
5.22 \\
4.94\end{array}$ & $\begin{array}{l}9.12 \\
9.28 \\
9.48 \\
9.99 \\
9.79\end{array}$ & $\begin{array}{r}.99 \\
.99 \\
1.01 \\
1.02 \\
1.04\end{array}$ & $\begin{array}{l}.04 \\
.04 \\
.04 \\
.05 \\
.05\end{array}$ & $\begin{array}{l}1.87 \\
1.92 \\
2.30 \\
2.24 \\
2.02\end{array}$ & $\begin{array}{l}2.90 \\
2.96 \\
3.35 \\
3.31 \\
3.11\end{array}$ \\
\hline $\begin{array}{l}1880 \\
1881 \\
1882 \\
1883 \\
1884\end{array}$ & $\begin{array}{l}4.59 \\
4.59 \\
4.62 \\
4.63 \\
4.68\end{array}$ & $\begin{array}{l}.45 \\
.56 \\
.57 \\
.58 \\
.59\end{array}$ & $\begin{array}{l}4.99 \\
5.39 \\
5.05 \\
4.87 \\
4.88\end{array}$ & $\begin{array}{l}10.03 \\
10.54 \\
10.23 \\
10.08 \\
10.15\end{array}$ & $\begin{array}{l}1.05 \\
1.05 \\
1.05 \\
1.06 \\
1.07\end{array}$ & $\begin{array}{l}.09 \\
.10 \\
.10 \\
.11 \\
.11\end{array}$ & $\begin{array}{l}2.10 \\
2.40 \\
2.21 \\
2.15 \\
2.10\end{array}$ & $\begin{array}{l}3.23 \\
3.55 \\
3.36 \\
3.32 \\
3.28\end{array}$ \\
\hline $\begin{array}{l}1885 \\
1886 \\
1887 \\
1888 \\
1889\end{array}$ & $\begin{array}{l}4.74 \\
4.80 \\
4.86 \\
4.93 \\
4.99\end{array}$ & $\begin{array}{l}.58 \\
.64 \\
.67 \\
.69 \\
.72\end{array}$ & $\begin{array}{l}4.96 \\
5.18 \\
5.41 \\
5.53 \\
5.56\end{array}$ & $\begin{array}{l}10.27 \\
10.61 \\
10.94 \\
11.15 \\
11.27\end{array}$ & $\begin{array}{l}1.09 \\
1.10 \\
1.11 \\
1.13 \\
1.13\end{array}$ & $\begin{array}{l}.11 \\
.11 \\
.11 \\
.16 \\
.20\end{array}$ & $\begin{array}{l}2.15 \\
2.22 \\
2.33 \\
2.36 \\
2.29\end{array}$ & $\begin{array}{l}3.35 \\
3.43 \\
3.55 \\
3.65 \\
3.62\end{array}$ \\
\hline $\begin{array}{l}1890 \\
1891 \\
1892 \\
1893 \\
1894\end{array}$ & $\begin{array}{l}5.03 \\
5.09 \\
5.09 \\
5.13 \\
5.17\end{array}$ & $\begin{array}{l}.73 \\
.79 \\
.81 \\
.90 \\
.90\end{array}$ & $\begin{array}{l}5.62 \\
5.75 \\
5.72 \\
5.79 \\
5.68\end{array}$ & $\begin{array}{l}11.38 \\
11.63 \\
11.63 \\
11.82 \\
11.74\end{array}$ & $\begin{array}{l}1.13 \\
1.14 \\
1.15 \\
1.15 \\
1.16\end{array}$ & $\begin{array}{l}.20 \\
.20 \\
.19 \\
.24 \\
.26\end{array}$ & $\begin{array}{l}2.20 \\
2.24 \\
2.23 \\
2.33 \\
2.34\end{array}$ & $\begin{array}{l}3.54 \\
3.59 \\
3.57 \\
3.72 \\
3.76\end{array}$ \\
\hline $\begin{array}{l}1895 \\
1896 \\
1897 \\
1898 \\
1899\end{array}$ & $\begin{array}{l}5.18 \\
5.22 \\
5.31 \\
5.39 \\
5.42\end{array}$ & $\begin{array}{l}1.00 \\
1.03 \\
1.06 \\
1.07 \\
1.11\end{array}$ & $\begin{array}{l}5.86 \\
6.01 \\
6.02 \\
5.95 \\
5.88\end{array}$ & $\begin{array}{l}12.04 \\
12.27 \\
12.39 \\
12.41 \\
12.41\end{array}$ & $\begin{array}{l}1.17 \\
1.19 \\
1.21 \\
1.22 \\
1.22\end{array}$ & $\begin{array}{l}.26 \\
.26 \\
.27 \\
.28 \\
.29\end{array}$ & $\begin{array}{l}2.42 \\
2.58 \\
2.50 \\
2.47 \\
2.52\end{array}$ & $\begin{array}{l}3.86 \\
4.03 \\
3.98 \\
3.97 \\
4.03\end{array}$ \\
\hline $\begin{array}{l}1900 \\
1901 \\
1902 \\
1903 \\
1904\end{array}$ & $\begin{array}{l}5.44 \\
5.52 \\
5.55 \\
5.56 \\
5.60\end{array}$ & $\begin{array}{l}1.16 \\
1.19 \\
1.23 \\
1.30 \\
1.35\end{array}$ & $\begin{array}{l}5.70 \\
5.65 \\
5.72 \\
5.79 \\
5.90\end{array}$ & $\begin{array}{l}12.30 \\
12.36 \\
12.50 \\
12.65 \\
12.85\end{array}$ & $\begin{array}{l}1.22 \\
1.23 \\
1.24 \\
1.25 \\
1.27\end{array}$ & $\begin{array}{l}.29 \\
.30 \\
.31 \\
.32 \\
.33\end{array}$ & $\begin{array}{l}2.47 \\
2.41 \\
2.45 \\
2.52 \\
2.59\end{array}$ & $\begin{array}{l}3.98 \\
3.94 \\
3.99 \\
4.08 \\
4.19\end{array}$ \\
\hline $\begin{array}{l}1905 \\
1906 \\
1907 \\
1908 \\
1909\end{array}$ & $\begin{array}{l}5.60 \\
5.55 \\
5.56 \\
5.58 \\
5.09\end{array}$ & $\begin{array}{l}1.37 \\
1.45 \\
1.45 \\
1.54 \\
1.58\end{array}$ & $\begin{array}{l}5.94 \\
5.68 \\
5.60 \\
5.61 \\
5.72\end{array}$ & $\begin{array}{l}12.91 \\
12.68 \\
12.61 \\
12.73 \\
12.39\end{array}$ & $\begin{array}{l}1.28 \\
1.27 \\
1.27 \\
1.28 \\
1.29\end{array}$ & $\begin{array}{l}.33 \\
.35 \\
.35 \\
.37 \\
.39\end{array}$ & $\begin{array}{l}2.62 \\
2.49 \\
2.44 \\
2.37 \\
2.38\end{array}$ & $\begin{array}{l}4.22 \\
4.11 \\
4.07 \\
4.02 \\
4.06\end{array}$ \\
\hline $\begin{array}{l}1910 \\
1911 \\
1912 \\
1913\end{array}$ & $\begin{array}{l}5.13 \\
5.22 \\
5.26 \\
5.37\end{array}$ & $\begin{array}{l}1.68 \\
1.75 \\
1.85 \\
1.90\end{array}$ & $\begin{array}{l}5.93 \\
6.32 \\
6.41 \\
6.56\end{array}$ & $\begin{array}{l}12.74 \\
13.29 \\
13.52 \\
13.83\end{array}$ & $\begin{array}{l}1.30 \\
1.33 \\
1.35 \\
1.42\end{array}$ & $\begin{array}{l}.40 \\
.41 \\
.42 \\
.43\end{array}$ & $\begin{array}{l}2.46 \\
2.57 \\
2.56 \\
2.64\end{array}$ & $\begin{array}{l}4.16 \\
4.32 \\
4.33 \\
4.49\end{array}$ \\
\hline
\end{tabular}

Source: see text. 
Appendix C: National construction, 1861-1913: value added at 1911 prices (million lire)

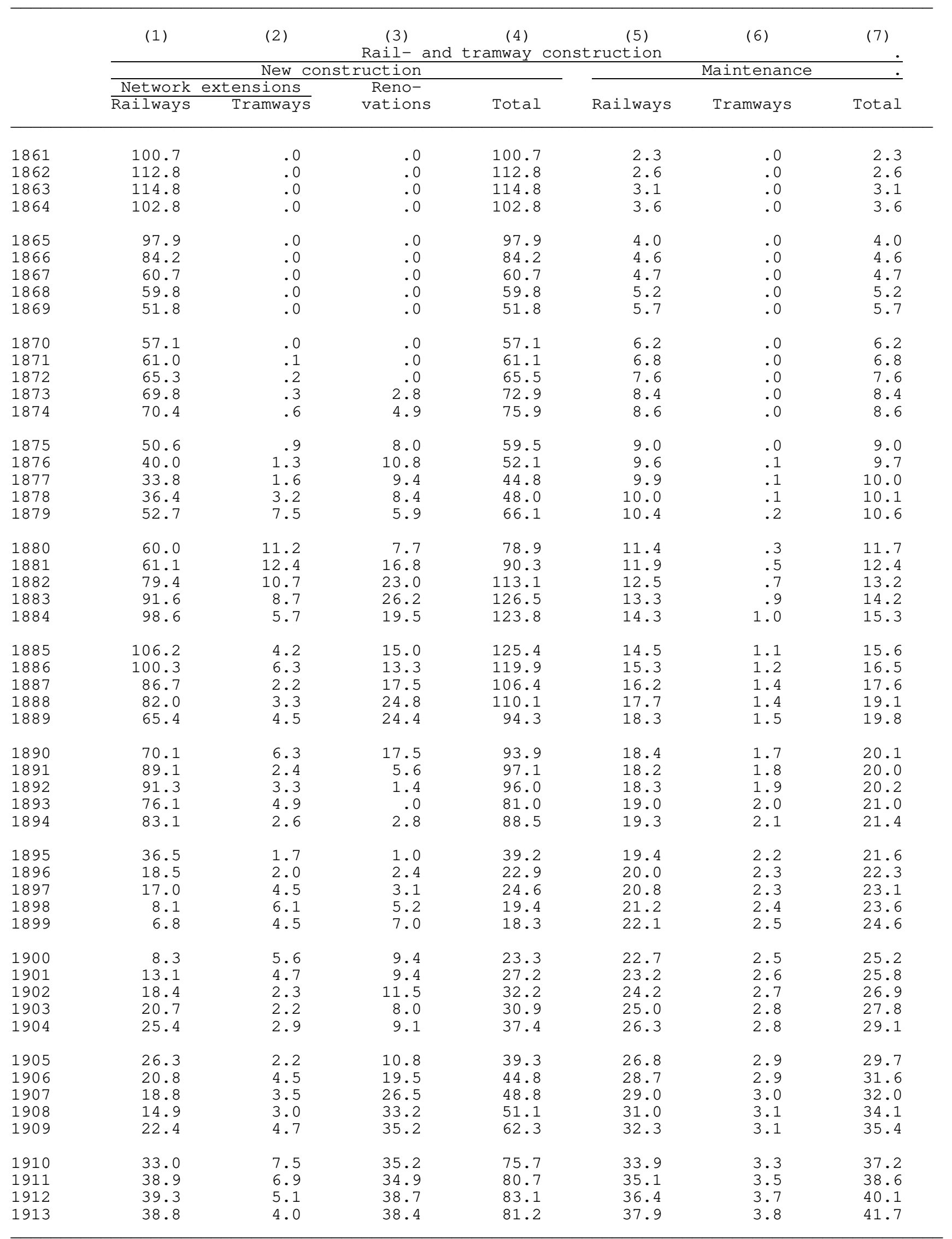




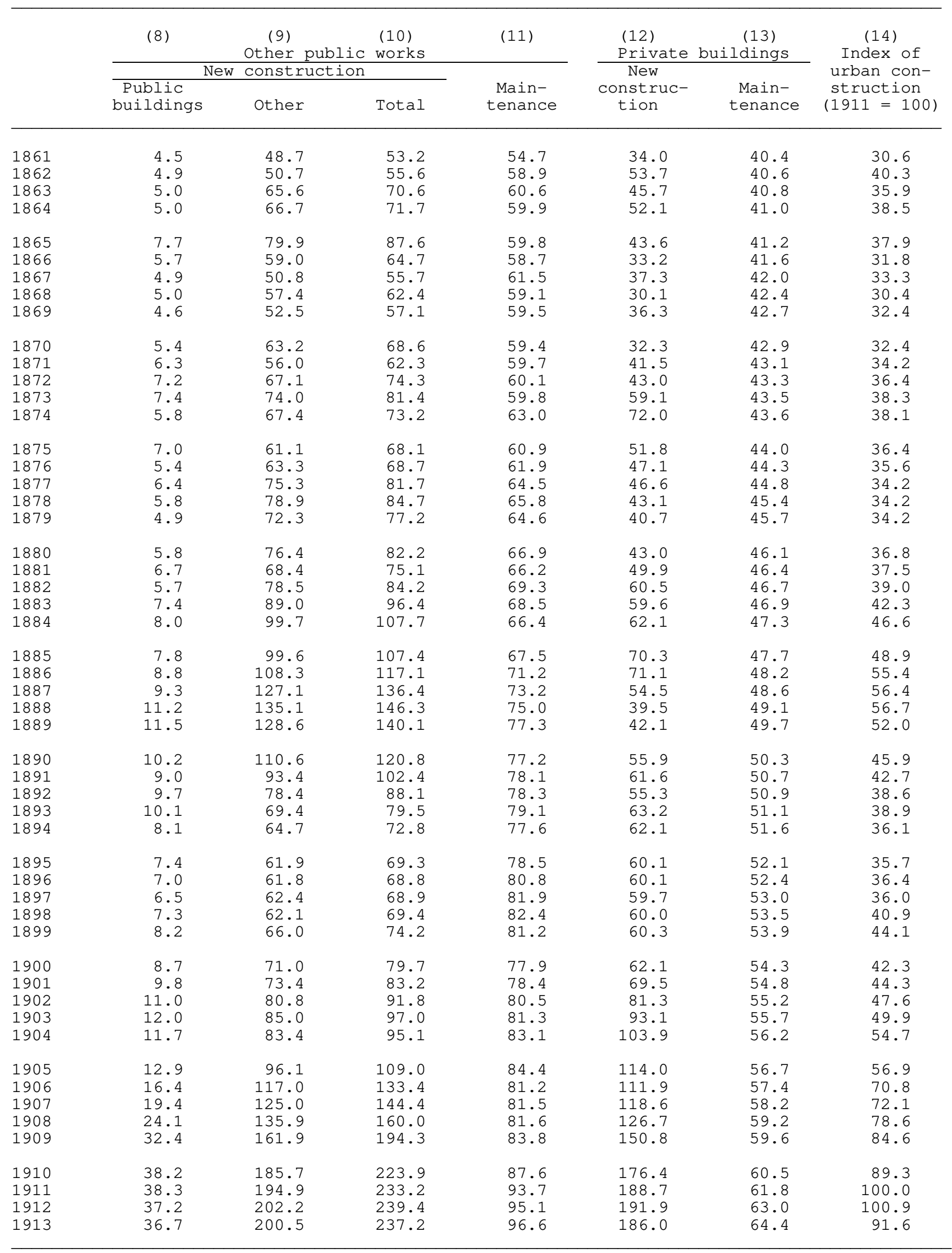




\begin{tabular}{|c|c|c|c|c|}
\hline & (15) & $\begin{array}{l}(16) \\
(16)\end{array}$ & $\begin{array}{c}(17) \\
\text { (17) }\end{array}$ & $(18)$ \\
\hline & $\begin{array}{c}\text { Railways } \\
\text { and } \\
\text { tramways }\end{array}$ & $\begin{array}{r}\text { Other } \\
\text { public } \\
\text { works }\end{array}$ & $\begin{array}{c}\text { Private } \\
\text { buil- } \\
\text { dings }\end{array}$ & Total \\
\hline $\begin{array}{l}1861 \\
1862 \\
1863 \\
1864\end{array}$ & $\begin{array}{l}103.0 \\
115.4 \\
117.9 \\
106.4\end{array}$ & $\begin{array}{l}107 \cdot 9 \\
114.5 \\
131.2 \\
131.6\end{array}$ & $\begin{array}{l}74.4 \\
94.3 \\
86.5 \\
93.1\end{array}$ & $\begin{array}{l}285.3 \\
324.2 \\
335.6 \\
331.1\end{array}$ \\
\hline $\begin{array}{l}1865 \\
1866 \\
1867 \\
1868 \\
1869\end{array}$ & $\begin{array}{r}101.9 \\
88.8 \\
65.4 \\
65.0 \\
57.5\end{array}$ & $\begin{array}{l}147.4 \\
123.4 \\
117.2 \\
121.5 \\
116.6\end{array}$ & $\begin{array}{l}84.8 \\
74.8 \\
79.3 \\
72.5 \\
79.0\end{array}$ & $\begin{array}{l}334.1 \\
287.0 \\
261.9 \\
259.0 \\
253.1\end{array}$ \\
\hline $\begin{array}{l}1870 \\
1871 \\
1872 \\
1873 \\
1874\end{array}$ & $\begin{array}{l}63.3 \\
67.9 \\
73.1 \\
81.3 \\
84.5\end{array}$ & $\begin{array}{l}128.0 \\
122.0 \\
134.4 \\
141.2 \\
136.2\end{array}$ & $\begin{array}{r}75.2 \\
84.6 \\
86.3 \\
102.6 \\
115.6\end{array}$ & $\begin{array}{l}266.5 \\
274.5 \\
293.8 \\
325.1 \\
336.3\end{array}$ \\
\hline $\begin{array}{l}1875 \\
1876 \\
1877 \\
1878 \\
1879\end{array}$ & $\begin{array}{l}68.5 \\
61.8 \\
54.8 \\
58.1 \\
76.7\end{array}$ & $\begin{array}{l}129.0 \\
130.6 \\
146.2 \\
150.5 \\
141.8\end{array}$ & $\begin{array}{l}95.8 \\
91.4 \\
91.4 \\
88.5 \\
86.4\end{array}$ & $\begin{array}{l}293.3 \\
283.8 \\
292.4 \\
297.1 \\
304.9\end{array}$ \\
\hline $\begin{array}{l}1880 \\
1881 \\
1882 \\
1883 \\
1884\end{array}$ & $\begin{array}{r}90.6 \\
102.7 \\
126.3 \\
140.7 \\
139.1\end{array}$ & $\begin{array}{l}149.1 \\
141.3 \\
153.5 \\
164.9 \\
174.1\end{array}$ & $\begin{array}{r}89.1 \\
96.3 \\
107.2 \\
106.5 \\
109.4\end{array}$ & $\begin{array}{l}328.8 \\
340.3 \\
387.0 \\
412.1 \\
422.6\end{array}$ \\
\hline $\begin{array}{l}1885 \\
1886 \\
1887 \\
1888 \\
1889\end{array}$ & $\begin{array}{l}141.0 \\
136.4 \\
124.0 \\
129.2 \\
114.1\end{array}$ & $\begin{array}{l}174.9 \\
188 \cdot 3 \\
209.6 \\
221.3 \\
217.4\end{array}$ & $\begin{array}{r}118.0 \\
119.3 \\
103.1 \\
88.6 \\
91.8\end{array}$ & $\begin{array}{l}433.9 \\
444.0 \\
436.7 \\
439.1 \\
423.3\end{array}$ \\
\hline $\begin{array}{l}1890 \\
1891 \\
1892 \\
1893 \\
1894\end{array}$ & $\begin{array}{l}114.0 \\
117.1 \\
116.2 \\
102.0 \\
109.9\end{array}$ & $\begin{array}{l}198.0 \\
180.5 \\
166.4 \\
158.6 \\
150.4\end{array}$ & $\begin{array}{l}106.2 \\
112.3 \\
106.2 \\
114.3 \\
113.7\end{array}$ & $\begin{array}{l}418.2 \\
409.9 \\
388.8 \\
374.9 \\
374.0\end{array}$ \\
\hline $\begin{array}{l}1895 \\
1896 \\
1897 \\
1898 \\
1899\end{array}$ & $\begin{array}{l}60.8 \\
45.2 \\
47.7 \\
43.0 \\
42.9\end{array}$ & $\begin{array}{l}147.8 \\
149.6 \\
150.8 \\
151.8 \\
155.4\end{array}$ & $\begin{array}{l}112.2 \\
112.5 \\
112.7 \\
113.5 \\
114.2\end{array}$ & $\begin{array}{l}320.8 \\
307.3 \\
311.2 \\
308.3 \\
312.5\end{array}$ \\
\hline $\begin{array}{l}1900 \\
1901 \\
1902 \\
1903 \\
1904\end{array}$ & $\begin{array}{l}48.5 \\
53.0 \\
59.1 \\
58.7 \\
66.5\end{array}$ & $\begin{array}{l}157.6 \\
161.6 \\
172.3 \\
178.3 \\
178.2\end{array}$ & $\begin{array}{l}116.4 \\
124.3 \\
136.5 \\
148.8 \\
160.1\end{array}$ & $\begin{array}{l}322.5 \\
338.9 \\
367.9 \\
385.8 \\
404.8\end{array}$ \\
\hline $\begin{array}{l}1905 \\
1906 \\
1907 \\
1908 \\
1909\end{array}$ & $\begin{array}{l}69.0 \\
76.4 \\
80.8 \\
85.2 \\
97.7\end{array}$ & $\begin{array}{l}193.4 \\
214.6 \\
225.9 \\
241.6 \\
278.1\end{array}$ & $\begin{array}{l}170.7 \\
169.3 \\
176.8 \\
185.9 \\
210.4\end{array}$ & $\begin{array}{l}433.1 \\
460.3 \\
483.5 \\
512.7 \\
586.2\end{array}$ \\
\hline $\begin{array}{l}1910 \\
1911 \\
1912 \\
1913\end{array}$ & $\begin{array}{l}112 \cdot 9 \\
119 \cdot 3 \\
123 \cdot 2 \\
122.9\end{array}$ & $\begin{array}{l}311.5 \\
326.9 \\
334.5 \\
333.8\end{array}$ & $\begin{array}{l}236.9 \\
250.5 \\
254.9 \\
250.4\end{array}$ & $\begin{array}{l}661.3 \\
696.7 \\
712.6 \\
707.1\end{array}$ \\
\hline
\end{tabular}

Source: see text. 